Development and applications of theoretical algorithms for simulations of materials at extreme conditions

\author{
Igor Mosyagin
}

10 LINKÖPINGS

Linköping University

Department of Physics, Chemistry and Biology

Theory and Modeling

SE-581 83 Linköping, Sweden

Linköping 2017 
The cover image is inspired by the melding of solid state physics and computer science.

During the course of research underlying this thesis, Igor Mosyagin was enrolled in Agora Materiae, a multidisciplinary doctoral program at Linköping University, Sweden.

(c) Igor Mosyagin

ISBN 978-91-7685-543-0

ISSN 0345-7524

Typeset using $\mathrm{ET}_{\mathrm{E}} \mathrm{X}$, cursing, and unicorns

Printed by LiU-Tryck, Linköping 2017 
to my family especially to Oscar! The real cat, the best cat 



\begin{abstract}
Materials at extreme conditions exhibit properties that differ substantially from ambient conditions. High pressure and high temperature expose anharmonic, non-linear behavior, and can provoke phase transitions among other effects. Experimental setups to study that sort of effects are typically costly and experiments themselves are laborious. It is common to apply theoretical techniques in order to provide a road-map for experimental research. In this thesis I cover computational algorithms based on first-principles calculations for high-temperature and high-pressure conditions. The two thoroughly described algorithms are: 1) the free energy studies using temperature-dependent effective potential method (TDEP), and 2) a higher-order elastic constants calculation procedure. The algorithms are described in an easy to follow manner with motivation for every step covered.

The Free energy calculation algorithm is demonstrated with applications to hexagonal close-packed Iron at the conditions close to the inner Earth Core's. The algorithm of elastic constants calculation is demonstrated with application to Molybdenum, Tantalum, and Niobium. Other projects included in the thesis are the study of effects of van der Waals corrections on the graphite and diamond equations of state.
\end{abstract}

\title{
Svensk sammanfattning
}

Material vid extrema förhållanden uppvisar egenskaper som skiljer sig avsevärt från omgivningsförhållanden. Högt tryck och hög temperatur exponera anharmonicity, icke-linjärt beteende, och kan framkalla fasövergångar bland andra effekter. Experimentella uppställningar för att studera denna typ av effekter är vanligtvis dyra och experiment själva är mödosam. Det är vanligt att tillämpa teoretiska metoder för att ge en färdplan för experimentell forskning. I denna avhandling täcker jag beräkningsalgoritmer baserat på första principer beräkningar för hög temperatur och högt tryck. De två grundligt beskrivna algoritmer är: 1) den fria energin studier med temperaturberoende effektiv potentiell metod (TDEP), och 2) en högre ordning elastiska konstantberäkningsproceduren. Algoritmerna beskrivs i en lätt att följa sätt med motivation för varje steg som omfattas.

Den fria energiberäkningsalgoritm visas med program till hexagonal tätpackad järn på villkoren nära jordens inre kärna. Algoritmen av elastiska konstanter beräkning demonstreras med tillämpning till molybden, tantal, och niob. Andra projekt som ingår i avhandlingen är effekterna av van der Waals-korrigeringar på tillståndsekvation och elastiska konstanter i grafit och diamant. 



\section{Acknowledgment}

This thesis is a summary of my PhD studies in the Theoretical physics group at Linköping University. The last five years would not be possible without a certain number of people that I would like to acknowledge below.

First and foremost, I am thankful to Igor Abrikosov for providing scientific guidance and for tolerating my stubbornness over the last five years. I would like to also thank my co-supervisor Sergei Simak for his helpful advises, wit and suggestions, all delivered with clarity and atomic-like precision.

During my first two years as a PhD student I was a teaching assistant for an undergraduate course on Quantum mechanics. I enjoyed that a lot, though I am not sure that the students partaking the course had as much fun. Nevertheless, I would like to thank Magnus Boman for this opportunity. I definitely learned a few pedagogical tricks from you.

Recurrent meetings with my mentor, professor Carl-Fredrik Mandenius, and follow-up meetings with professor Per-Olof Holtz proven to be very helpful with keeping my motivation, especially at times when I assumed there were none left. Thank you for helping me to stay on course.

Understanding complicated concepts is an inevitable gritty part of doing research. It is helpful to be able to bore someone from time to time with shaky questions and dumb assumptions. I am grateful to Andrey Lugovskoy and Alexey Tal for providing much-needed company when I needed to bounce ideas.

Given the fact that modern research is impossible without collaborations, I would like to thank all of my coauthors who provided necessary feedback and input for all projects included in this thesis.

Since naming every other possible person would take too much space, and I would not like to left anyone out, I finally would like to acknowledge the lunch group for detailed, weird and nerdy discussions and a few friends outside of university that I made during the last five years. You know who you are. Thank you for putting up with me.

The combined managerial superpowers of Irina Yakimenko, Jessica Gidby, Lejla Krönback, Anna-Karin Stål, and Lise-Lotte Lönndahl Ragnar would be enough to crush a few mountains. With their help, any administrative issue I encountered was nothing but a piece of cake. Speaking of cakes, no Slav can survive long enough without grandma's cooking, and I am no exception to the rule. Бабушка! Спасибо за пироги и поддержку. Без тебя я бы не справился.

Finally, I would like to acknowledge "Shut Up \& Write Tuesdays" community, Headspace app, the Ubersleep folks, Dima Pechkeen, and beeminder service for helping me being constent with my writing.

Igor Mosyagin

Linköping, March 2017 



\section{Contents}

1 Introduction 13

1.1 Computer simulations in theoretical physics . . . . . . . . . . . . . . . . . . 13

1.1.1 Computer simulations and experiment . . . . . . . . . . . . . . . . . . . . 14

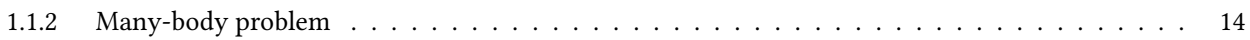

1.1 .3 Parallel computing . . . . . . . . . . . . . . . . . . . . . . . . . 15

1.1 .4 Latencies and access times . . . . . . . . . . . . . . . . . . . . . . 17

1.1.5 Heuristic algorithms and Data science . . . . . . . . . . . . . . . . . . . 17

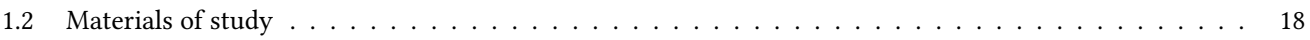

1.2 .1 Iron and Titanium $\ldots \ldots \ldots \ldots \ldots \ldots \ldots \ldots$

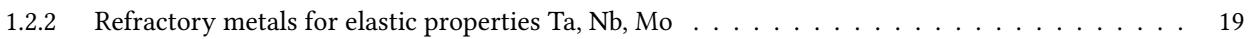

1.2 .3 Graphite . . . . . . . . . . . . . . . . . . . . . . . . . 19

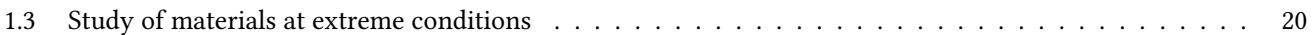

2 Methods 23

2.1 Density functional theory . . . . . . . . . . . . . . . . . . . . . . . . . 24

2.1 Hohenberg-Kohn theorems . . . . . . . . . . . . . . . . . . . . . 25

2.1 .2 Kohn-Sham theory . . . . . . . . . . . . . . . . . . . . . . . 25

2.1 .3 Exchange-correlation . . . . . . . . . . . . . . . . . . . . 26

2.1.4 Density Functional Theory limitations . . . . . . . . . . . . . . . . . . . . 28

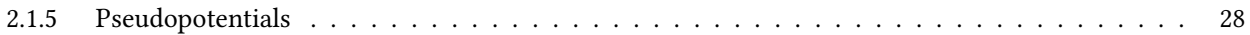

2.1.6 Building pseudopotential . . . . . . . . . . . . . . . . . . . . . . . . . . 29

2.1 .7 Projected augmented waves method . . . . . . . . . . . . . . . . . 31

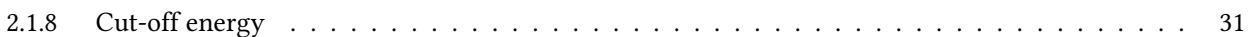

2.1 .9 Integration over the Brillouin zone . . . . . . . . . . . . . . . . . . . . . 31

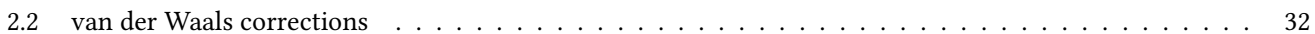

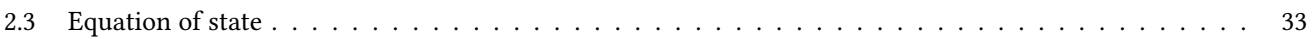

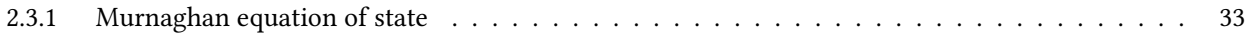

2.3.2 Birch-Murnaghan equation of state . . . . . . . . . . . . . . . . . . . . . 34

2.3.3 Rose-Vinet equation of state . . . . . . . . . . . . . . . . . . . . . . . 34

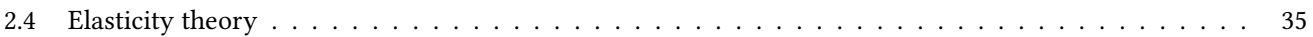

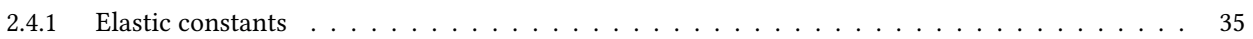

2.4.2 Calculating elastic constants using infinitesimal deformations method . . . . . . . . . . 35

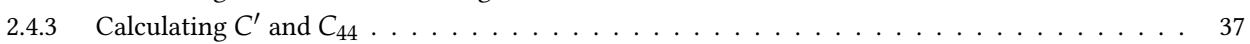

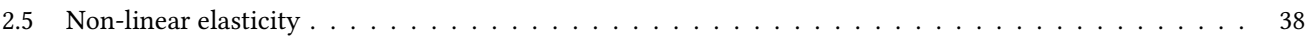


2.5.1 Thermodynamic definition of elastic constants . . . . . . . . . . . . . . . 38

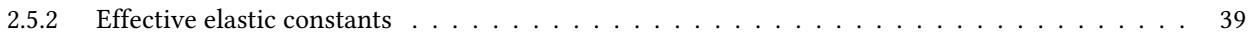

2.5.3 Hydrostatic pressure . . . . . . . . . . . . . . . . . . . . . . . 39

2.5.4 Applications of higher-order elastic constants . . . . . . . . . . . . . . . . . . 40

2.6 Molecular dynamics . . . . . . . . . . . . . . . . . . . . . . . . . . 41

2.7 Temperature-dependent effective potential method . . . . . . . . . . . . . . . . . 43

2.7.1 Free energy calculations within TDEP method . . . . . . . . . . . . . . . . . . 43

2.7.2 Helmholtz free energy components within TDEP method . . . . . . . . . . . . . . 45

2.7.3 Interpolation of force constants matrix components . . . . . . . . . . . . . . . . 46

3 Algorithms $\quad 40$

3.1 Computational algorithm for temperature-dependent effective potential method . . . . . . . . . . . 49

3.2 Higher-order elastic constants computational algorithm . . . . . . . . . . . . . . . . . 52

4 Applications for studies of specific materials and properties $\mathbf{5 7}$

4.1 Equation of state of hexagonal close-packed iron at $2000 \mathrm{~K} \ldots \ldots \ldots$

4.2 Elastic properties of $\mathrm{Nb}, \mathrm{Ta}, \mathrm{Mo} \ldots \ldots \ldots \ldots \ldots$

4.2.1 Elastic constants of body-centered cubic Molybdenum under pressure . . . . . . . . . . . 58

4.2.2 Elastic constants of body-centered cubic Tantalum under pressure . . . . . . . . . . . . 60

4.2.3 Elastic constants of body-centered cubic Niobium under pressure . . . . . . . . . . . . . 63

4.3 Effect of van der Waals corrections on the description of graphite and diamond . . . . . . . . . . . 66

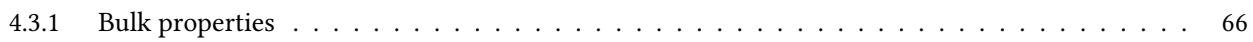

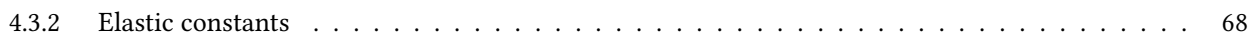

5 Conclusion $\quad 73$

$\begin{array}{ll}\text { Papers and contributions } & 85\end{array}$ 
The answer to this is very simple. It was a joke.

It had to be a number, an ordinary, smallish number, and I chose that one.

Binary representations, base thirteen, Tibetan monks are all complete nonsense.

I sat at my desk, stared into the garden and thought « 42 will do». I typed it out.

End of story.

Douglas Adams 



\section{Introduction}

This thesis deals with a subject of crossover between theoretical physics and computer science. Since both those concepts are humongous, I find it's important to outline which particular parts of them my work is related to.

\section{Computer simulations in theoretical physics}

First, as often happens when discussing «theoretical physics», it irks me that the common assumption that one is talking about high-energy particle physics and string theory. This feeds the narcissism of those who choose that particular sub-field, but theoretical physics is much more than that. American physics society publishes five main Physics Review journals (from A to the E) ${ }^{1}$, and roughly half of the papers published in each one are theory papers. Only Physical Review D is concerned with string theory and high-energy particle physics, which is roughly $20 \%$ of all theory papers in those 5 journals.

Second, the field of theoretical physics is no longer relies that heavy on office supplies and the pure pen and paper physicist are rare. There are tons of interesting complex systems which exhibits phenomena that just cannot be studied by hand. Most of the time, the word "complex" in this context is just a substitute for "many". And when many-body problems are discussed, whether it is quantum mechanics or celestial mechanics, many can be as low as 3 to crush any attempts to describe the system and its interaction analytically and expand such description beyond a few well-behaved special cases.. Realistical systems, studied by the field of theoretical materials science, are quite dense but even when they are not, the amount of total interacting elements in the system is tremendous. Let us consider highschool chemistry, in particular a definition of an Avogadro number ${ }^{2}$. The number itself is outside humanly imaginable region $\left(6 \times 10^{23}\right)$, but one's sanity is saved by the conveniently naming and, therefore, unclear feeling for a «single mole» quantity in its definition. To connect this number with reality, let's recall the other concept from high-school chemistry class, also introduced by Avogadro, namely molar volume of an ideal gas at a standard conditions -22.4 liters. Now, imagine a row of 6 one-liter milk packages side-by-side which should not be that hard if you ever been to a grocery
${ }^{1}$ I consider "specialized physics journal here", so I am ommiting Physical Review Letters, Physical Review X or recently announced Physics Review Materials

\footnotetext{
${ }^{2}$ «A total amount of atoms (or molecules) contained in an amount of substance given by one mole»
} 
store: that is roughly one-fourth of that 22.4 liters. If that volume on the shell were occupied by an ideal gas, there would be around $10^{23}$ single molecules on the shelf. If the same volume is occupied by something more dense than an ideal gas (like milk), the number of particles would be even higher. The thing is that even the smallest humanly imaginable volume of, say, a grand of sand, would contain so many atoms and electrons in it, that it would be absolutely impossible to study them using analytical methods. Of course, there are methods to alleviate some of those limitations when symmetry and periodicity of studied system is taken into account, but still most of the modern-days theoretical physics relies heavily on numerical simulations, complicated computational methods and supercomputers.

Not being familiar with computational instruments is just throwing away an extremely valuable tool.

Computer simulations and experiment

Considering computer simulations throughout this thesis, I would like to compare them to a real experiment, similarly to the idea expressed by Frenkel and Smith [1]. Most of the real-life experiments have three distinct phases, namely preparation of the sample, modifying conditions for the measurement, and, finally, gathering statistics. The next phase - data analysis - has nothing specific for the experiment comparing to theory, aside from the outdated secret society agenda to use confusing units of measurement.

For example, in the case of atomic force microscope, sample preparation would be cutting sample and fixing it on a piezoactuator. The conditionsmodifying phase could be heating of the sample or applying magnetic field for a certain amount of time, and then waiting some time for the system to reach desired condition. The third phase would be a measurement itself, which assumes that nothing else is changing in the environment.

Theoretical computer simulations are similar to that, and especially so in the case of molecular dynamics (MD) simulations. Any molecular dynamic simulation is an iterative process that consists of three distinct phases: building simulation cell, equilibration, and sampling. In Paper 3 I discuss a method that allows to minimize the equilibration phase, leaving more useful data for sampling (measurement), which is achieved by making "sample preparation" step more elaborate.

\subsubsection{Many-body problem}

In quantum mechanics, we often solve the Schrödinger equation [2] for one or many particles. In its most basic time-independent form it can be written like this:

$$
\hat{H} \Psi=E \Psi,
$$


where $\hat{H}$ is a Hamiltonian operator, acting on a system in a state described as $\Psi$, and $E$ is an eigenvalue of such operator, representing energy of the said state $\Psi$. Hamiltonian operator describes the interactions, and $\Psi$ is generally a specially constructed wave function. Hamiltonian $\hat{H}$ is itself a linear combination of operators, and for most of the problems those operators are based on partial derivatives or special functions. When there are $\mathrm{N}$ electrons interacting via Coulomb potential, that means that a $3 \mathrm{~N}$ dimensional partial differential equation need to be solved to describe the system. Since we also need to take into account the fermionic nature of electrons, the wavefunction needs to be anti-symmetric with respect to the interchange of any electronic pair. Due to the fact that $N$ for a more or less interesting system is big, even modern numerical methods cannot straightforwardly solve such systems, so there is a demand for approximations.

Solving the Schrödinger equation for a single electron moving in a vicinity of an ion and therefore affected by its atomic potential is already a complicated problem. Not only one has to solve partial differential equation, you should also take into account the fact that the atomic potential is very strong when the distance is small and very weak when you are far away. The solution would be a wave function with varying length scale, that requires a non-regular discrete grid which is finer close to the nuclei. The problem gets increasingly more complicated as more particles become involved. There are several approaches to this quantum many-electron problem, and the one that I use in this thesis is a Density Functional Theory $^{3}$, developed in $1965[3,4]$. Overall, the chain of approximations allows to end with a matrix eigenvalue problem, with a size of the matrix depending on the number of atoms. The set of methods that solve such problems in an efficient and parallel manner lies in the realms of computer science and parallel linear algebra.

\subsubsection{Parallel computing}

Over the last few decades, the invention of transistor and the tools to minimize the circuits lead to a rapid growth of computational power. The most common way to show this progress is a Moore's law [5], which was formulated in the same 1965, and states that the number of transistors in a dense integrated circuit doubles approximately every two years. Graphically, from Moore's law it follows that the logarithm of a system's performance plotted with respect to time would be linear, see Fig 1.1.

However, if we take a look into top systems descriptions, we find out that the CPU frequency of these machines might be even lower than the one of a typical laptop. For example, the Sunway TaihuLight complex [6], the №1 machine in the latest edition of a list, is built on a processors with a clock rate of just $1.45 \mathrm{GHz}$. Peak performance of this machine is estimated to be $93014.6 \mathrm{TFlop} / \mathrm{s}$, which is roughly 3 times higher than the peak

\footnotetext{
${ }^{3}$ In principle, density functional theory is not an approximation, but an exact theory. However, on practice it can be used only with a few additional approximations, discussed in section 2.1
} 
Figure 1.1: Exponential growth of supercomputing power as recorded by the TOP500 list [7]. Moore's law still holds despite the quinquennial claims by computer journalists that it should retire «a few months from now» since around 2000.

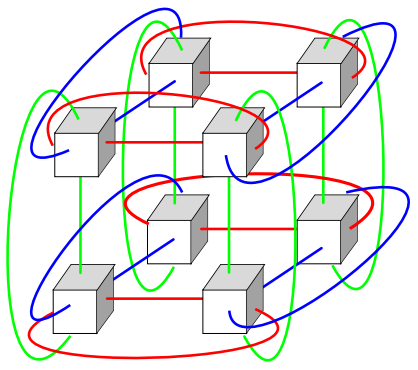

Figure 1.2: 3-dimensional torus interconnect can be used to minimize inter-node network distance, providing better connectivity and overall performance.

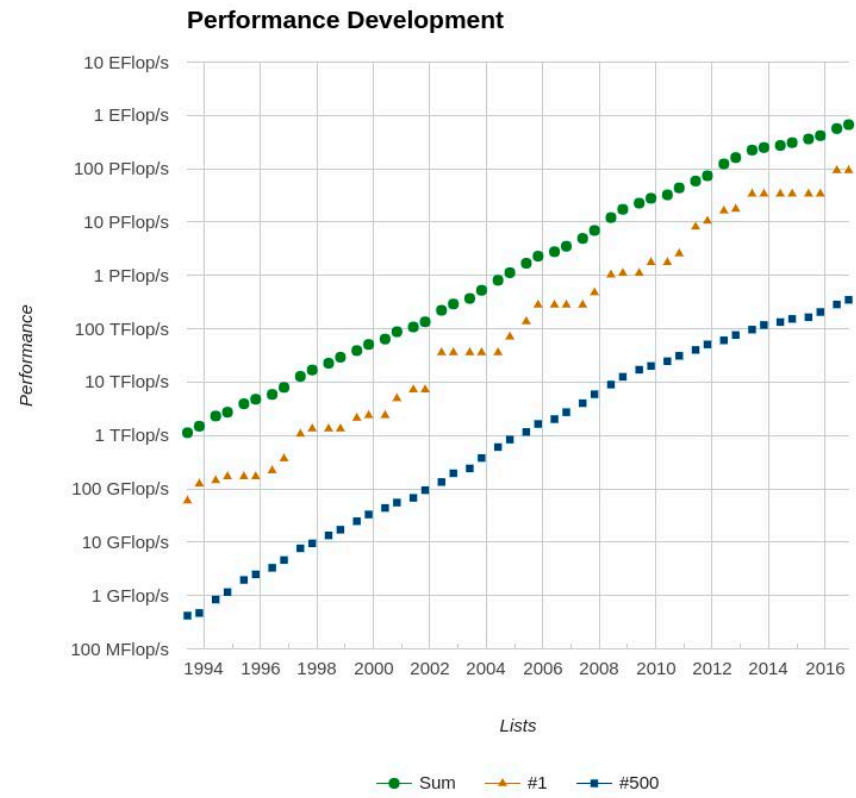

performance of the next machine in the same list.

The standard performance estimation procedure uses a LINPACK test based on a popular LAPACK library [8], which provides linear algebra routines for high-performance environment. The systems are graded by their performance in solving a set of linear equations $\|\mathcal{A}\| \cdot\|x\|=\|b\|$, where $\|\mathcal{A}\|$ is a dense random matrix. The decision to base the grading on such test has been criticized since the very beginning of a TOP500 project, but since it is transferable between various systems and architectures, it is considered to be one of the most reliable options.

Inevitably, such metric affected the way a modern high-performance systems are constructed, with emphasis on scalability rather than single-node performance. The supercomputer cluster based around a slower processors might outperform the one with a faster CPUs by having more nodes, providing better inter-node connectivity, and using faster network. Moreover, since lower clock rates generally drain less power, these solutions implicitly leave lesser $\mathrm{CO}_{2}$ footprint, which is one of the important ethical concerns of any scientist, at least according to an Uppsala Code [9].

It boils down to the idea that in order to utilize the state-of-the-art supercomputers, one should take care of developing a software that scales well and can utilize as much parallelization as it can get. Unfortunately, not every problem can be easily parallelized and even when it is, it's hard to get 
good scaling with respect to the system size. When the problem scales with size with ease, or with little effort, it is considered to be "embarrassingly parallel". An example of such problem could be the one where the problem can be sliced to multiple independet pieces that can be computed in parallel and merged back into one at the later stages.

\subsubsection{Latencies and access times}

The connectivity between the individual machines is not the only thing that is important to take into account when developing massively parallel software. The way the program utilizes memory usage is also important, because if the system is faster than the memory then it is being inefficient both computation- and energy-wise. Table 1.1 shows the typical memory access latencies and some routine system operations, along with corresponding time-spans, scaled to a human-relatable intervals.

\begin{tabular}{lrlrl}
\hline Event & \multicolumn{2}{c}{ Latency } & \multicolumn{2}{c}{ Scaled } \\
\hline 1 CPU cycle & 0.3 & $\mathrm{~ns}$ & 1 & $\mathrm{~s}$ \\
Level 1 cache access & 0.9 & $\mathrm{~ns}$ & 3 & $\mathrm{~s}$ \\
Level 2 cache access & 2.8 & $\mathrm{~ns}$ & 9 & $\mathrm{~s}$ \\
Level 3 cache access & 12.9 & $\mathrm{~ns}$ & 43 & $\mathrm{~s}$ \\
DRAM memory access & 120 & $\mathrm{~ns}$ & 6 & min \\
Solid-state disk I/O & $50-150$ & $\mu \mathrm{s}$ & $2-6$ & days \\
Rotational disk I/O & $1-10$ & $\mathrm{~ms}$ & $1-12$ & months \\
Internet: Europe to US & 81 & $\mathrm{~ms}$ & 8 & years \\
TCP packet re-transmit & $1-3$ & $\mathrm{~s}$ & $105-317$ & years \\
Physical system reboot & 5 & $\mathrm{~m}$ & 32 & millennia \\
\hline
\end{tabular}

It is important then to develop methods that stay localized and self-sustained as much as possible during the computational run. Every additional call for external data, be it a stored memory variable or a data synchronization with another machine would slow down the active part of the computation, increasing the time-to-solution. Functional programming paradigm takes this idea to extreme and considers such actions to be "side effects", with many functional languages developed in a ways that promote the avoidance of such actions. Typically, programs written in functional programming language scale exceptionally good, but tend to be intrinsicly slow.

\subsubsection{Heuristic algorithms and Data science}

The recent rise of Data science and machine learning (DS/ML) methods lead to revisit analysis phase and the data processing techniques used in the scientific community, enforcing cross-pollination of ideas between those research fields. There been a few works on implementing various machine learning techniques for high-throughput research [11,12], and a few obser-
Table 1.1: Example Time Scale of System Latencies [10] 
vations that, in a sense, many-body problem in quantum mechanics can be reformulated in a terms of dimensional reduction and feature extraction. This makes it possible to apply machine learning techniques, such as neural networks and reinforced learning $[13,14]$, to the typical problems of materials science. The problem of deep learning can itself be understood in terms of renormalization group theory [15], which leads to an interesting conclusion that a computer program that recognizes cats in a video uses the same logic as one that solves complex statistical physics problem. Neural networks has been shown to be applicable as a bridge between $a b$ initio molecular dynamics and classical molecular dynamics simulations [16].

The idea behind a high-throughput analysis is to use statistical techniques on a sufficiently big database of results in order to suggest new compounds and conditions for a target variable. Suggested points in this configuration space can then be verified by a full-scale calculation, and added to the original database improving future predictions. There are a few projects that help combining and storing calculation results and provide an interface for data manipulation [17-19].

\subsection{Materials of study}

\subsubsection{Iron and Titanium}

Iron is one of the most abundant materials on Earth and the Earth core is considered to be composed of iron, iron-nickel alloy and some light elements. Despite being one of the most theoretically studied metal, there are still gaps in understanding its behavior under Earth Core conditions of extreme pressure and high temperature. Experiments carried out with laserheated diamond anvil cell $[20,21]$ disagree with results from shock waves experiments [22-24]. Furthermore, theoretical results vary a lot across all the possible properties such as crystal structure, thermal conductivity, melting temperature and elasticity [25-32].

Much of these uncertainties come from the fact that accurate temperatureincluding simulations were impossible up until recently. Most of ab initio structural stability calculations were carried at $0 \mathrm{~K}$ [32-41].

In my research, I applied temperature-dependent effective potential method $[42,43]$ to calculate equation of state of hexagonal close-packed Iron and high pressure and high temperature conditions. Systematic step-bystep description of such calculation is the main topic of Paper 3.

Titanium and titanium-based alloys are highly important technological systems with a lot of applications in aerospace, bio-medicine and cutting tool coatings to name a few. Body-centered cubic phase of Titanium is unstable at $T=0 \mathrm{~K}$, but becomes stable at higher temperatures. Therefore it is an excellent system for temperature-dependent effective potential method, which I used to calculate phonon dispersion relations of body- 
centered cubic Titanium and study dynamical stabilization of this phase in papers 4 and 5 .

\subsubsection{Refractory metals for elastic properties $\mathrm{Ta}, \mathrm{Nb}, \mathrm{Mo}$}

Transition metals, including molybdenum, are often present in high pressure experiments [44-48] as a reference samples. When studying structural transitions under pressures comparable with material's elastic constants, the stability of high pressure phase is ensured by non-linear elasticity of a material. Vanadium is known to exhibit a body centered cubic to rhombohedral phase transition under the pressure of $69 \mathrm{GPa}$ at room temperature, revealed by synchrotron X-ray diffraction experiments [49]. The interesting thing about this transition that, instead of transitioning into a structure with higher symmetry, the final structure appears to be less symmetric than the initial one. This prompted to investigate surrounding materials in the periodic table, namely tantalum, niobium and molybdenum. In order to study non-linear elasticity, a framework based on a finite strain deformations was developed. The corresponding procedure is presented in Paper 1 where the resulting elastic constants of second- and third- order of bodycentered Molybdenum are presented. Elastic properties of Tantalum and Niobium are discussed in section 4.2 .

\subsubsection{Graphite}

Graphite is one of the two most common forms of carbon, the other being diamond. While diamond is reputably hard and is a common component in cutting tools, graphite is a soft material used in lubrication and as pencil lead. The difference in properties of these two materials is connected to their respective crystalline structures. Carbon atoms in diamond are $s p^{3}$ hybridized in a tetrahedral geometry, which gives rise to the exceptional hardness. Contrary, graphite represents a stack of weakly interacting planar sheets of $s p^{2}$-hybridized carbon atoms. The anisotropic bonding means that these layers can glide easily over each other, which leads to a macroscopic softness.

Honeycomb structure of graphite is a well-known testbed for theoretical studies. While the free energy difference these two structures is only $2.9 \mathrm{~kJ} \mathrm{~mol}^{-1}$, it is very difficult to convert graphite to diamond. For this transition to occur, one requires temperatures higher than $1700 \mathrm{~K}$ and pressures above $12 \mathrm{GPa}$. Understanding the transition mechanism on the atomic scale is a very difficult problem [14,51-53].

Theoretical studies of graphite are complicated by the nature of interlayer interactions. In order to describe graphite structure, one needs a way to simultaneously take into account both strong covalent bonds $\left(s p^{2}\right.$ and $s p^{3}$ ) and weak dispersion interactions.

The random phase approximation [54] accounts for non-local dispersive

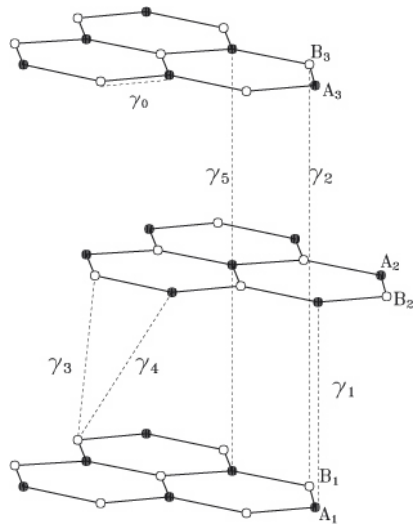

Figure 1.3: The geometric structure of the AB-stacked graphite (3 layers depicted). $\gamma_{0}$ is the intralayer interaction and the $\gamma_{i}$ indicate the various interlayer interactions [50] 
interactions without relying on fitting parameters or error cancellation, but it is computationally expensive, which prohibits its use for large system sizes. This size limitation, in turn, rules out phase nucleation studies. Moreover, random phase approximation is not that great at describing covalent interactions [55]. Diffusive quantum Monte Carlo can describe both dispersive and covalent bonds but the computational costs are even higher [56].

A good compromise between accuracy and computational complexity can be found by correcting the exchange correlation functional of density functional theory. The covalent bonds are generally well described by standard local and semi-local exchange and correlation approximations. Those methods are inherently unable to describe the intrinsically non-local dispersion interactions. The development of several functionals which aim to include such weak van der Waal (vdW) forces in the recent years allowed to accurately describe the structure of graphite [57], and other 2D solids, as well as the physisorption of organic molecules on surfaces.

However, very few studies attempted to determine if such methods can simultaneously describe covalent bonding, in particular at high pressures. If one wishes to study phase transitions of carbon from graphite to a diamond phase, it is important that any corrections introduced to describe the weakly bound structure of graphite does not affect the structural parameters of diamond.

In paper 2 I present a comparative study of most used van der Waals corrections applied to those two allotropes of Carbon.

\subsection{Study of materials at extreme conditions}

Since there is a sub-string "extreme conditions" in a title of this thesis, I feel the need to clarify what is meant by that. This expression typically have a different meaning depending on who are you talking to. Staying in the realm of the materials science, it usually stands for high temperature and/or high pressure. The magnitude for both of those thermodynamic quantities to be considered "high" is essentially emotional. High temperature would probably have the same meaning for both experimentalist and theoretician, and is, in my view, typically related to temperatures higher than $1000 \mathrm{~K}$. High pressure, on the other hand, could be as high as several GPa to provide experimentally extreme conditions, but that is next to an equilibrium when material is studied theoretically. When I mention high pressure throughout this thesis, I imply pressures comparable with the value of a bulk modulus of investigated materials, which is on the order of a hundred GPa. While it is possible to reach multiple hundreds of GPa pressure in the state-ofthe-art experimental setups [45-47], reaching these levels of compression requires complicated assemblies and could be the sole reason for the experiment behind it. The measurement of elastic constants at extreme static compression is a challenging task that contains some uncertainties [58]. 
This makes theoretically obtained elastic constants trends under pressure a valuable addition to the knowledge about studied materials. On the other hand, compression is just an input variable for a theoretical simulation. While too high compression would inevitably produce numerical artifacts, it is not uncommon to safely carry out simulations at $300 \mathrm{GPa}$ and higher. 



\section{Methods}

The development of quantum mechanics in XX century allowed to describe physical systems on atomic level and beyond. The main equation of quantum mechanics - Schrödinger equation in its time-independent (also known as "static") form looks mostly harmless:

$$
\hat{H} \tilde{\Psi}=E \tilde{\Psi},
$$

where $\tilde{\Psi}$ is a wavefunction, describing the state of the system with energy $E$. All physics that is accounted in the model is hidden in a special operator form in Hamiltonian $\hat{H}$.

Any condensed phase of matter is a complex system consisting of nuclei, electrons, and interactions among them. It can be imagined as a bunch of atoms close enough to each other in such a way that their wavefunctions start to overlap. The total wavefunction of a system with $N$ identical electrons and $N_{n}$ nuclei in general, would depend on a total of $N+N_{n}$ coordinates $^{1}$ :

$$
\tilde{\Psi}=\tilde{\Psi}\left(R_{1}, \ldots R_{N_{n}}, r_{1}, \ldots r_{N}\right),
$$

${ }^{1}$ assuming non-relativistic case and no spin dependency

where $R_{J}$ is ionic radius-vector of corresponding ion $j, 1 \leq J \leq N_{n}$, and $r_{i}$ is an electronic radius-vector of electron $i, 1 \leq i \leq N$. Assuming nuclei have corresponding masses $M_{J}$ and charges $Z_{J}$, the Hamiltonian of the system can be expressed with a following set of equations:

$$
\begin{gathered}
\hat{H}=\hat{H}_{e}+\hat{H}_{n}+\frac{1}{2} \sum_{i=1}^{N} \sum_{J=1}^{N_{n}} \frac{Z_{J}}{\left|\mathbf{R}_{J}-\mathbf{r}_{i}\right|} . \\
\hat{H}_{e}=\sum_{i=1}^{N}-\frac{\nabla_{i}^{2}}{2}+\frac{1}{2} \sum_{i \neq j}^{N} \frac{1}{\left|\mathbf{r}_{i}-\mathbf{r}_{j}\right|}, \\
\hat{H}_{n}=\sum_{J=1}^{N_{n}}-\frac{\nabla_{j}^{2}}{2 M_{J}}+\frac{1}{2} \sum_{I \neq J}^{N_{n}} \frac{Z_{I} Z_{J}}{\left|\mathbf{R}_{I}-\mathbf{R}_{J}\right|} .
\end{gathered}
$$

where $\hat{H}_{n}$ and $\hat{H}_{e}$ are the Hamiltonians of nuclei- and electron-only subsystems; $\mathbf{r}_{i}$ and $\mathbf{r}_{j}$ are the corresponding positions of electrons $i$ and $j ; \mathbf{R}_{J}$ and $\mathbf{R}_{J}$ are the corresponding positions of any two nuclei $I$ and $J$. First terms on the right hand side of eqs. (2.4) and (2.5) represent the total kinetic 
${ }^{2}$ for example, a lone Hydrogen molecule

${ }^{3}$ sometimes referenced as "adiabatic approximation" energy of either electronic or nuclei subsystem; the second terms represent Coulomb interaction. The last term in (2.3) represents electron-nuclei Coulomb interaction.

Both classical and quantum mechanics are fail to describe many body systems analytically except for boring cases ${ }^{2}$. In fact, the direct use of (2.3), (2.4), and (2.5) in (2.1) gives equation too complex to solve even numerically, so approximations are used. One of them is a Born-Oppenheimer approximation $^{3}$, that allows to decouple the movement of the electrons and the movement of nuclei. This is reasonable due to the fact that the mass $M$ of a typical nuclei is much larger than the mass $m$ of an electron: $m / M \approx 10^{-4} \ll 1$.

Effectively, what Born-Oppenheimer approximation allows us to do is to decouple electronic and ionic wavefunctions and rewrite the systems' total wavefunction as follows:

$$
\tilde{\Psi}\left(R_{1}, \ldots R_{N_{n}}, r_{1}, \ldots r_{N}\right)=\Phi\left(R_{1}, \ldots R_{N_{n}}\right) \Psi\left(R_{1}, \ldots R_{N_{n}}, r_{1}, \ldots r_{N}\right)
$$

This would in turn lead to an easier Hamiltonian

$$
\hat{H}=-\frac{1}{2} \sum_{i=1}^{N}-\nabla_{i}^{2}+\frac{1}{2} \sum_{i \neq j}^{N} \frac{1}{\left|\mathbf{r}_{i}-\mathbf{r}_{j}\right|}+\frac{1}{2} \sum_{i=1}^{N} \sum_{J=1}^{N_{n}} \frac{Z_{J}}{\left|\mathbf{R}_{J}-\mathbf{r}_{i}\right|} .
$$

\subsection{Density functional theory}

Calculating the electronic properties of solid is not a trivial task, given the fact that the atomic nuclei and the electrons constitute a complex manybody problem. All theories that deal with these sort of problems start by adopting the adiabatic (Born-Oppenheimer) approximation, that allows to evaluate electronic properties while neglecting the movement of the atomic nuclei. Since the electrons are much lighter than nuclei, it is a pretty solid claim. The approximation allows to focus on the electrons, but it is in itself still quite a challenging problem. In a material, electrons interact through the means of Coulomb interaction both with each other as well as with the positive atomic nuclei. The interaction with atomic nuclei is straightforward even though it is still hard. However, interaction between electrons is a mess that is impossible to untangle without introducing further approximations. Endeavors to estimate electronic dispersion in solids and create a model of electron-electron interaction to calculate total energy of various systems dates back to Thomas-Fermi model [59,60] and Slater method [61]. Both provided a base for $a b$ initio description of complex electronic structures, further developed and formulated by Kohn and Sham [3]. This theory provides the means to calculate the total energy of solids, relying only on electronic density $n(\mathbf{r})$ as its central variable. 
Density functional theory is based on two theorems by Hohenberg and Kohn, formulated in 1964 [4].

Theorem 1. For any system of interacting electrons in an external potential $V_{\mathrm{xc}}(\mathbf{r})$, that potential $V_{\mathrm{xc}}(\mathbf{r})$ can be defined, up to a numeric constant, from ground state electronic density $n_{0}(\mathbf{r})$.

If we know the potential, then we know systems' Hamiltonian, and we can find wavefunction of the system. Therefore all properties of the system are defined from its ground state electronic density $n_{0}(\mathbf{r})$. Theorem 1 postulates connection between external potential and ground state electronic density. It does not provide any way of finding external potential using electronic density alone.

Theorem 2. For any external potential there exist a universal energy functional $E[n]$ that can be expressed through electronic density $n(\mathbf{r})$. For any specific $V_{\text {ext }}(\mathbf{r})$, the exact ground state energy would minimize this functional and the corresponding electronic density $n(\mathbf{r})$ which gives this minimum is the exact ground state electronic density $n_{0}(\mathbf{r})$.

Therefore, in order to find exact ground state energy and ground state electronic density, it is sufficient to know functional $E[n]$. Using these two theorems, we conclude that for any external potential we can always find electronic density (and, therefore, ground state energy) by minimizing that functional $E[n]$.

Energy functional $E[n]$ for specific external potential $v_{\text {ext }}$, using adiabatic approximation, can be expressed in the following general form:

$$
E[n]=T[n]+E_{H}[n]+E_{\mathrm{xc}}[n]+\int v_{\mathrm{ext}}(\mathbf{r}) n(\mathbf{r}) d \mathbf{r},
$$

where $E_{H}$ is an electron-electron Coulomb interaction, also known as Hartree term, the $T[n]$ is a kinetic energy, and the rightmost term accounts for interaction in the external potential. These terms are investigated further in the following subsections.

\subsubsection{Kohn-Sham theory}

So far it was only discussed that it is possible to reduce the big amount of variables that describe electrons to one variable - electronic density. I have not said anything on how to find it and how to find corresponding energy. In 1965 Kohn and Sham suggested that it is possible to escape many-body problem by introducing a new auxiliary system that would be easier to describe and resolve. The main idea is to replace many-body interacting system with a non-interacting system that would have the same ground state energy, see Fig. 2.1. If this "auxiliary" system would 
then have the same ground state electronic density as the "real" system, then Hohenberg-Kohn theorems guarantee that we would have the correct energy of the system. Below I follow Martin's book on electronic structure calculations [62] to introduce several important equations.

The Kohn-Sham equation is the Schrödinger equation of an auxiliary system of non-interacting particles. The said particles generate the same density as any given system of interacting particles. The equation is defined by an effective potential that surrounds non-interacting particles:

$$
\left(-\frac{\hbar^{2}}{2 m} \nabla^{2}+v_{\text {eff }}(\mathbf{r})\right) \psi_{k}(\mathbf{r})=\varepsilon_{k} \psi_{k}(\mathbf{r}),
$$

where $\varepsilon_{k}$ is the energy of the corresponding Kohn-Sham orbital $\psi_{k}$. The density of a system that contains $N$-particles is expressed as $n(\mathbf{r})$ :

$$
n(\mathbf{r})=\sum_{k=1}^{N}\left|\psi_{k}(\mathbf{r})\right|^{2},
$$

and the energy of this system is expressed in a form of a functional of this density. I rewrite the equation (2.8), expanding each term:

$$
\begin{aligned}
E[n] & =\underbrace{\sum_{k=1}^{N} \int \psi_{k}^{*}(\mathbf{r})\left(-\frac{\hbar^{2}}{2 m} \nabla^{2}\right) \psi_{k}(\mathbf{r}) d \mathbf{r}} \\
& +\frac{e^{2} \frac{e^{2} \int\left[\mathbf{r} \int d \mathbf{r}^{\prime} \frac{n(\mathbf{r}) n\left(\mathbf{r}^{\prime}\right)}{\left|\mathbf{r}-\mathbf{r}^{\prime}\right|}\right.}{E_{H}[n]}}{} \\
& +E_{\mathrm{xc}}[n]+\int v_{\mathrm{ext}}(\mathbf{r}) n(\mathbf{r}) d \mathbf{r},
\end{aligned}
$$

and the term $E_{\mathrm{xc}}$ is the exchange-correlation energy, sometimes described as "everything else". The equations (2.9), (2.10), and (2.11) can be solved by varying the total energy expression with respect to a set of orbitals and necessary constraints. This provides an expression for the effective potential:

$$
v_{\text {eff }}(\mathbf{r})=v_{\text {ext }}(\mathbf{r})+e^{2} \int \mathbf{r}^{\prime} \frac{n\left(\mathbf{r}^{\prime}\right)}{\left|\mathbf{r}-\mathbf{r}^{\prime}\right|}+\underbrace{\frac{\delta E_{\mathrm{xc}}[n]}{\delta n[\mathbf{r}]}}_{v_{\mathrm{xc}}(\mathbf{r})},
$$

where the exchange-correlation potential $v_{\mathrm{xc}}(\mathbf{r})$, along with its parent expression for the exchange-correlation energy $E_{\mathrm{xc}}$, are representing the unknowns of the Kohn-Sham density functional theory.

\section{Exchange-correlation}

Since the general form of exchange-correlation functional is unknown, we have to use approximations. The two most common approximations for exchange-correlation functional are local density approximation (LDA) and general gradient approximation (GGA). 
Local Density Approximation was suggested by Kohn and Sham [3] and is the easiest approximation: exchange-correlation term for the case of LDA is formulated as follows:

$$
E_{\mathrm{xc}}^{\mathrm{LDA}}[n]=\int \varepsilon_{\mathrm{xc}}[n(\mathbf{r})] n(\mathbf{r}) \mathrm{d}^{3} r,
$$

where $\varepsilon_{\mathrm{xc}}$ is the exchange-correlation energy density of the uniform electronic gas with density $n(\mathbf{r})$. Energy density $\varepsilon_{\mathrm{xc}}$ is obtained from parametrisation of electronic gas modeled using Monte-Carlo simulation. As one can see from (2.13), different parametrisations of $\varepsilon_{\mathrm{xc}}$ could lead to a different result in the actual calculations. For single-atom systems LDA can be bad, because single atomic systems are significantly different from uniform electronic gas, but for solids this approximation is used for more than 40 years and appears to be a relatively good choice, mainly due to error compensation.

For atoms and molecules, LDA underestimates exchange interaction by $10 \%$ and overestimate correlation by $200-300 \%$. For many systems, contribution from exchange correlation is by an order of magnitude bigger than the contribution from correlation, therefore combined exchangecorrelation error is in a range of $7 \%$.

Local density approximation is a great tool to study solid state, except for band gap studies in semiconductors, that can disappear completely and in general are badly reproduced. One of the well-known problem with LDA is a prediction of the ground state of iron: LDA gives nonmagnetic fcc lattice, while the stable structure is bcc ferromagnetic [64].

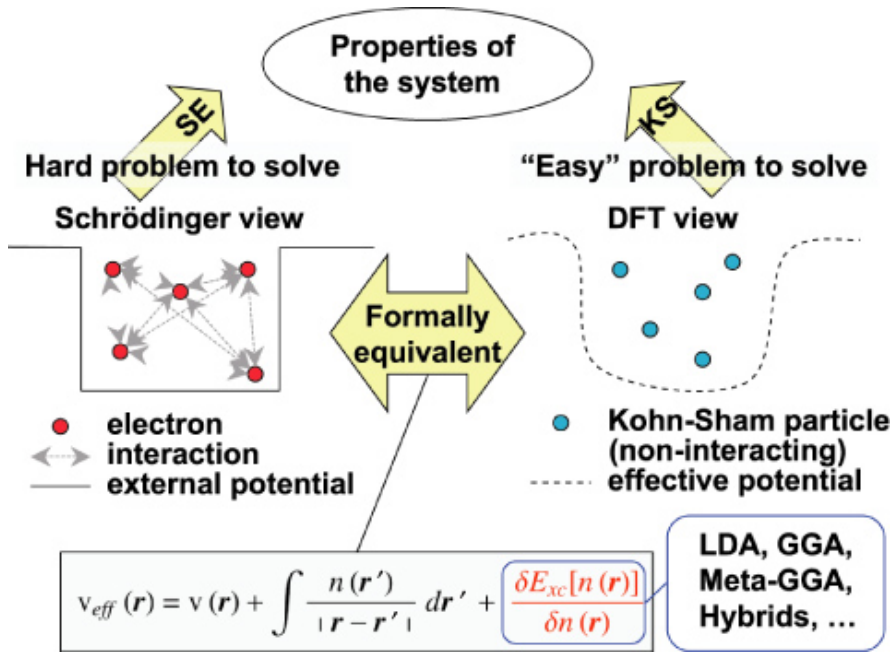

Figure 2.1: Main idea of DFT is to replace one complex many-body problem with $3 \mathrm{~N}$ degrees of freedom with multiple non-interacting problems in external effective potential with 3 degrees of freedom each [63]. Theory provides formal equivalence in this transition and therefore ensures that it is possible to find all the interesting properties of the investigated system. 
Generalized gradient approximation allows to solve some of the problems of LDA by including explicit density gradient dependence. Such approximation can be expressed in its generalized form as follows:

$$
E_{\mathrm{xC}}^{\mathrm{GGA}}[n]=\int f[n(\mathbf{r}), \nabla n(\mathbf{r})] n(\mathbf{r}) \mathrm{d}^{3} r .
$$

Since there is no unambiguous way to choose how $E_{\mathrm{xc}}^{\mathrm{GGA}}[n]$ behaves with respect to $\nabla n(\mathbf{r})$, there exist many ways to define (2.14), each with its own pros and cons. In general all of them focus to solve problems that arise from using LDA. In some cases inclusion of gradient of electronic density into exchange-correlation might lead to additional complications in terms of choosing parameters of computational experiment. Generally speaking, it makes sense to carry out initial tests to find which approximation gives better results if one has the way to compare it with some reference. One of the most proficient implementations for GGA was developed by Perdew, Burke and Erzerhof and generally referenced as PBE-GGA [65, 66].

\section{Density Functional Theory limitations}

Density functional theory is a wide-spread and successful theory. It is used to describe metals, alloys, semiconductors, molecules, nanoclusters and surface effects. On one hand, Hohenberg-Sham theorems affect only ground state energy of the investigated systems, and auxiliary Kohn-Sham particles are only ensure that the resulting electronic density is correct for the ground state. Therefore, DFT calculations are valid only for ground state properties. However, Hohenberg-Kohn theorems tell us that once we obtain electronic density of the system's ground state, we also define the system's Hamiltonian. In this sense, we can investigate not only the ground state properties but excited states as well.

In real calculations we cannot know the full Hamiltonian of the system due to the used approximations. In most cases when studying excited states it is necessary to expand the theory so that applied approximations could be carried over to the systems of interest.

It is important to note that the density functional theory itself does not provide any means to study matter based on a lone electronic property. Although it was shown above that it the knowledge of electronic density is sufficient for this, the relations between characteristics of electronic densities and any other electronic properties are unknown, even for the simplest question, for example, whether the system is metallic or an insulator.

\subsubsection{Pseudopotentials}

The main idea of a pseudopotential method in condensed matter theory consists, again, in substitution of one complex problem with another, less complicated one. We substitute strong Coulomb interaction between nuclei 
and strongly-coupled core electrons with effective ionic potential that interacts with the electrons in the valence band. Therefore, when studying electronic properties of solids, every electronic state of atoms is in one of three groups: it's either a core state, a valence state or a semi-core state. The distribution between those three groups occurs as follows:

1. Core states are localized and do not participate in atomic bonds formation;

2. Valence states are non-localized and therefore participate in atomic bonds formation;

3. Semi-core states are not participating in atomic bonds formation but lie high enough to interact with valence states.

In most of the cases, it is sufficient to consider only valence states. However, occasionally the system might be in such conditions that it is necessary to include semi-core states in calculation with the same rights as valence states. Core states are described using frozen core approximation, that considers all core states as an isolated singular atom, contribution from which can be calculated only once and this way the effect of core states is easier to account for [62].

Valence electrons in crystal in most cases behave as almost free electrons, since the external potential is mostly smooth. One of the common ways to simplify calculations is to expand wavefunctions in a series of plane waves. This allows to achieve high computation efficiency with rather simple implementation, since the total number of plane waves required is relatively low. However, close to the nuclei, the potential diverges and the wavefunction become a fast oscillating function. Therefore one requires a lot of plane waves to describe it with sufficient precision, which significantly lowers calculation efficiency. One of the ways of solving this is the pseudopotential method. Core electrons are considered similar to the way they would in a stand-alone atom, and interaction between valence electrons and core electrons is described approximately with effective potential. This way, valence electrons far from nuclei are in smooth potential, but when it's close, we solve the issue of constructing complex wavefunction. Obviously, construction of a good pseudopotential is a non-trivial task.

\subsubsection{Building pseudopotential}

When using frozen core approximation, the wavefunctions of electrons in valence state should satisfy Schrödinger equation:

$$
\hat{H} \psi_{\mathbf{k}}^{v}=\epsilon_{\mathbf{k}}^{v} \psi_{\mathbf{k}}^{v}
$$

The valence wavefunction can be expanded in a series, similar to the 
orthogonal plane wave method:

$$
\psi_{\mathbf{k}}^{v}=\tilde{\psi}_{\mathbf{k}}^{v}-\sum_{c}\left[\int \mathrm{d}^{3} r^{\prime} \psi_{\mathbf{k}}^{c *}\left(\mathbf{r}^{\prime}\right) \tilde{\varphi}_{\mathbf{k}}^{v}\left(\mathbf{r}^{\prime}\right)\right] \psi_{\mathbf{k}}^{c}(\mathbf{r}),
$$

where $\psi_{\mathbf{k}}^{c}(\mathbf{r})$ are core states, and $\tilde{\psi}_{\mathbf{k}}^{v}-$ smooth part of wave function of valence electron that can be expanded in a plane waves:

$$
\tilde{\psi}_{\mathbf{k}}^{v}(\mathbf{r})=\sum_{\mathbf{k}^{\prime}} c_{\mathbf{k}^{\prime}} e^{i\left(\mathbf{k}+\mathbf{k}^{\prime}\right) \mathbf{r}} .
$$

If we now take (2.17) and plug it in (2.15), we can obtain the following expression:

$$
\left(\hat{H}+\hat{V}^{\prime}\right) \tilde{\psi}_{\mathbf{k}}^{v}=\varepsilon_{\mathbf{k}}^{v} \tilde{\psi}_{\mathbf{k}^{\prime}}^{v}
$$

where potential energy operator $\hat{V}^{\prime}$ is defined in such a way that

$$
\hat{V}^{\prime} \phi=\sum_{c}\left(\varepsilon_{\mathbf{k}}^{v}-\varepsilon_{\mathbf{k}}^{c}\right)\left[\int \mathrm{d}^{3} r^{\prime} \Psi_{\mathbf{k}}^{c *} \phi\right] \Psi_{\mathbf{k}}^{c}
$$

Therefore we changed wavefunction of valence electrons to a more smooth version that, however, gives the exact same eigenvalues. It is important to note that the pseudopotential in expression (2.19) is only one of the possible ways to solve this problem. What's important that any other method would have its own benefits and shortcomings, but should give the same eigenvalues $[67,68]$.

Pseudopotential is defined as a sum of real periodic potential $\hat{V}$ and auxiliary potential $\hat{V}^{\prime}$ :

$$
\hat{V}^{\mathrm{ps}}=\hat{V}+\hat{V}^{\prime} .
$$

Potential constructed in such way is smooth and weak, which is exactly what we were aiming for. It is complex and non-local, though. It also worth noting that in general smooth functions $\tilde{\psi}_{\mathbf{k}}^{v}$ are not orthonormal [62].

Constructed potential should be well-tested before it is used for real calculations of solids. To ensure that the potential gives good result, it is

${ }^{4} \mathrm{~A}$ peculiar issue with this approach is that some materials are better explained theoretically than the others due to the fact that there is more experimental data available to compare with. Example of such materials are Silicon and Aluminium common to compare the results obtained using constructed potential with the available experimental values ${ }^{4}$. The other way is to compare with the result of all-electron calculation, which is possible only for those materials that can be described with an all-electron method. At first view, it looks like the use of experimental data is the best possible test for computer experiment, however it is worth to remember that experiments are also prone to multiple unknown factors that can affect the end result. On the other hand, All-electron methods in most of the cases suffer from the same shortcomings as pseudopotential methods.

High quality potential should not only describe the material properties under tested and constructed conditions, but also reproduce the properties of material if its chemical status was altered (for example, if the potential for a chemical element was generated for a uniform crystal, it should be 
applicable for studying compounds that feature said element). Pseudopotentials that has this property are known as transferable. One of the way to achieve transferability of a pseudopotential is an additional requirement for norm-conservation. A nice side-effect of such restriction would be the simplification of the method of construction of such pseudopotential due to additional restrictions for a smooth part of the wavefunction.

\subsection{7}

To calculate energies of investigated systems in this work I used projected augmented waves method, developed by Bloch [69] and implemented by a group of Kresse in a Vienna ab initio simulation package (VASP) [70-75]. This method in its base is similar to orthogonal projected waves method, but the potential is constructed in such a way that preserves full-electron wavefunction.

\section{Cut-off energy}

Due to the one-to-one relationship between the distance to the nuclei and a wavevector in $k$-space, it is convenient to introduce cut-off energy. Cutoff energy is the energy that defines maximum kinetic energy of a plane wave that can be in a plane waves basis set. Denoting cut-off energy as $E_{\text {cut }}$, all plane waves with their wavevectors $\mathbf{k}$ must satisfy the following relationship:

$$
|\mathbf{G}+\mathbf{k}|<G_{\text {cut }} ; \quad E_{\text {cut }}=\frac{\hbar^{2}}{2 m} G_{\text {cut }}^{2},
$$

where $\mathbf{G}$ is a translation basis in a reciprocal space.

In case when atoms of the system under investigation end up close to each other ${ }^{5}$, it is important to check that the calculated energy of the system does not depend on cut-off energy, which should be explicitly tested. When cut-off energy is increased, the total amount of plane waves is increased as well and therefore computation time is also increased.

\subsubsection{Integration over the Brillouin zone}

Due to symmetry considerations, geometrically crystals are expressed with basis and translation vectors. Imposed symmetry on the cell allows to determine (and it's a reasonable goal to do so) the most compact way for this representation. The most compact lattice in the reciprocal space constructed this way is called Brillouin zone. For many properties studied by $a b$ initio calculations ${ }^{6}$, we need to integrate in $k$-space over the Brillouin zone, as such property would be expressed as an average over this volume.
${ }^{5}$ which might happen for high compression values, i.e. when high external pressure is applied
${ }^{6}$ distribution of electrons in the energy bands, total energy, charge density, etc 
${ }^{7}$ for example, $25 \times 25 \times 9$ gives 5625 points. The exact number of $f_{i}(k)$ calculation performed depends on the imposed symmetry of the system and could be an order of magnitude lower
Assuming the property $f$ can be expressed as a function of $\mathbf{k}$, that is $f_{i}(\mathbf{k})$, where $i$ accounts for degeneration, we can write the following expression:

$$
\bar{f}_{i} \propto \frac{1}{\Omega_{\mathrm{BZ}}} \int_{\mathrm{BZ}} d \mathbf{k} f_{i}(\mathbf{k}),
$$

where $\Omega_{\mathrm{BZ}}$ is a volume of a Brillouin zone, that can be found using primitive cell volume $\Omega_{\text {cell }}$ in a real $d$-dimensional space: $\Omega_{\mathrm{BZ}}=\Omega_{\text {cell }} /(2 \pi)^{d}$. To evaluate (2.22) numerically, we go from an integration to a summation:

$$
\frac{1}{\Omega_{\mathrm{BZ}}} \int_{\mathrm{BZ}} d \mathbf{k} f_{i}(\mathbf{k}) \rightarrow \frac{1}{N_{k}} \sum_{k} f_{i}(\mathbf{k}),
$$

and therefore the question of finding the average $\bar{f}_{i}$ boils down to $k$-point mesh grid and its density. The common way to chose $k$-points for a system is a Monkhorst-Pack method [76], and the grid is usually referenced as $k_{x} \times k_{y} \times k_{z} \cdot{ }^{7}$

\section{2 van der Waals corrections}

Standard local approaches fail to describe intrinsically non-local van der Waals interactions. The common way to overcome this is to add an energy correction to the conventional Kohn-Sham DFT energy, based on the behavior of isolated atoms. The other approach is to correct dispersion correction using electron density via a non-local correlation energy. While the latter require no external parameters, the former approach is in itself easier for implementation and more efficient computationally. For these functionals, the total energy $E_{\text {total }}$ can be expressed as follows:

$$
E_{\text {total }}=E_{\mathrm{KS}-\mathrm{DFT}}+E_{\mathrm{disp}}
$$

where $E_{\text {disp }}$ is the energy correction and $E_{\mathrm{KS}-\mathrm{DFT}}$ is the conventional Kohn-Sham DFT energy.

In Paper 2, $E_{\text {disp }}$ was used with the following correction methods: DFT-D3 method of Grimme [77, 78], with both zero and Becke-Jonson damping [79-83] and Tkachenko-Scheffler method with and without selfconsistent screening $[84,85]$.

The two methods that are not based on post-calculation energy correction that were used in Paper 2 are rev-vdW-DF2 and optB86b [86-88]. The exchange correlation energy for these methods is given by:

$$
E_{\mathrm{xc}}=E_{\mathrm{x}}^{\mathrm{GGA}}+E_{\mathrm{c}}^{\mathrm{LDA}}+E_{\mathrm{c}}^{\mathrm{nl}}
$$

where $E_{\mathrm{X}}^{\mathrm{GGA}}$ is GGA exchange energy, and $E_{\mathrm{C}}^{\mathrm{LDA}}$ is the local correlation within LDA. The remaining term, $E_{\mathrm{c}}^{\mathrm{nl}}$, is a non-local correlation energy, based on model response function of interacting electron densities [89]. The difference between rev-vdW-DF2 and optB86b lies in $E_{\mathrm{x}}^{\mathrm{GGA}}$ term. For 
rev-vdW-DF2 the exchange energy is that of of revised Perdew, Burke and Erzerhof functional (revPBE) [65, 66, 90] (revPBE), while optB86b uses Becke86 functional [91] with revised parameters (B86b).

One of the most important properties of any material is its equation of state (EOS). This term typically references to a $P=f(V, T)$ dependence. Furthermore, it is common to carry out ab initio calculations under condition of $T=0 \mathrm{~K}$ (or $T=$ const in case of Molecular-Dynamics). Generally, for most of materials the difference in theoretically studied properties at $T=0 \mathrm{~K}$ and room temperature is not that significant.

If energy dependency $E(V)$ is known, $P(V)$ equation of state and bulk modulus $B$ can be obtained using partial derivatives with respect to volume:

$$
\begin{gathered}
P=\frac{\partial E}{\partial V} \\
B=-V \frac{\partial P}{\partial V}=V \frac{\partial^{2} E}{\partial V^{2}}
\end{gathered}
$$

Sometimes we are also interested in the bulk modulus derivative $B^{\prime}$

$$
B^{\prime}=\frac{\partial B}{\partial P}=-\frac{V}{B} \cdot \frac{\partial B}{\partial V}
$$

To obtain $P(V)$ it is common to do multiple calculations of system's energy at various volumes which gives us set of energy-volume pairs $\left\{V_{i}: E_{i}\right\}$. Fitting this curve would give us $E(V)$ as a smooth (hopefully) function. Taking numerical derivatives of this function allows to find pressure as a function of volume and bulk modulus of the system in question. As a model function for $E(V)$ there are a few commonly used, such as Birch-Murnaghan or Rose-Vinet equation of state. Using such semi-empiric equations of state allows to minimize the amount of necessary calculations and reduce the effect of numeric artifacts. Initial guess for fitting parameters is usually performed using basic spline or polynomial fit to the same set of energy-volume data points.

The dependency $P(V)$ can be compared with experimental equation of state. The lattice parameters corresponding to $V_{0}$ so that $P\left(V_{0}\right)=0$ are called equilibrium lattice parameters ${ }^{8}$.

\section{Murnaghan equation of state}

Murnaghan basic assumption is that $B_{0}^{\prime}=$ const: bulk modulus derivative at $P=0$ is considered to be constant and independent of pressure [92]. Using this as a boundary condition, integrating (2.28) gives $E(V)$. Ground state parameters - equilibrium volume $V_{0}$, bulk modulus $B_{0}$ and its
${ }^{8}$ Bulk modulus $B$ and its derivative $B^{\prime}$ at equilibrium volume $V_{0}$ are usually denoted as $B_{0}$ and $B_{0}^{\prime}$, correspondingly. Naturally, $B_{0}=B\left(V_{0}\right)$, and $B_{0}^{\prime}=B^{\prime}\left(V_{0}\right)$ 
derivative $B_{0}^{\prime}$ can be found once $E(V)$ is fitted:

$$
E(V)=E\left(V_{0}\right)+\frac{B_{0} V}{B_{0}^{\prime}}\left(\frac{\left(V_{0} / V\right)^{B_{0}^{\prime}}}{B_{0}^{\prime}-1}+1\right)-\frac{B_{0} V_{0}}{B_{0}^{\prime}-1}
$$

Due to the assumption of pressure-independent constant $B_{0}^{\prime}$, this equation of state is great at describing systems close to its equilibrium volume, but not so good when the volume of the system is significantly smaller than $V_{0}$.

\subsection{2}

Birch-Murnaghan equation of state

Francis Birch aimed for an equation of state that would be applicable for high pressure research [93]. He proposed a model pressure dependency that depends on variable $\xi=V_{0} / V$ up to third order in $\xi$ :

$$
P=\frac{3 B_{0}}{2}\left(\xi^{\frac{7}{3}}-\xi^{\frac{5}{3}}\right)\left[1+\frac{3}{4}\left(B_{0}^{\prime}-4\right)\left(\xi^{\frac{2}{3}}-1\right)\right]
$$

Integrating (2.30) according to (2.26), we obtain energy as a function of volume, with the same set of parameters:

$$
E(V)=E_{0}+\frac{9}{16} B_{0} V_{0}\left[\left(\xi^{\frac{2}{3}}-1\right)^{3} B_{0}^{\prime}-\left(\xi^{\frac{2}{3}}-1\right)^{2}\left(4 \xi^{\frac{2}{3}}-6\right)\right]
$$

\subsubsection{Rose-Vinet equation of state}

${ }^{9}$ Since zero is a finite number, the temperature in question could be $0 \mathrm{~K}$
Rose-Vinet equation of state [94] is a modification of Birch-Murnaghan EOS and allows to study materials under isothermic conditions. It assumes that $B_{0}, B_{0}^{\prime}$ and $V_{0}$ are taken at the same finite temperature ${ }^{9}$ and zero pressure at that conditions. Denoting $\phi=\left(\frac{V}{V_{0}}\right)^{1 / 3}$, it features the following $P(V)$ dependency:

$$
P(V)=3 B_{0}\left(\frac{1-\phi}{\phi^{2}}\right) \exp \left[\frac{3}{2}\left(B_{0}^{\prime}-1\right)(1-\phi)\right]
$$

Correspondingly, this equation of state has the following $E(V)$ expression:

$$
\begin{aligned}
E(V) & =E_{0}+\frac{2 B_{0} V_{0}}{\left(B_{0}^{\prime}-1\right)^{2}} \\
& \times\left\{2-\left[5+3 B_{0}^{\prime}(\phi-1)-3 \phi\right] \exp \left(-\frac{3}{2}\left(B_{0}^{\prime}-1\right)(\phi-1)\right)\right\}
\end{aligned}
$$

The way the Rose-Vinet equation of state introduces its parameters allows to study isothermal properties of the system under investigation.

While implementation of equation of state fitting program is a simple straightforward task, there are multiple tools to perform that already. In particular, all examined equations of state in this section are implemented in Atomic Simulations Environment [95], which is a set of python libraries for common materials science tasks. 
While bulk modulus deals with isotropically applied pressure, it is not the only way of applying stress to the system. When there are forces applied to the crystal, deformations arise. Deformations in turn lead to displacements. \\ Elastic constants}

Assume that a point $P$ with a radius-vector $\mathbf{x}$ in crystal becomes point $P^{\prime}$ with a radius-vector $\mathbf{x}^{\prime}$ due to applied deformation. The displacement vector $\mathbf{u}$ is defined in classical elasticity theory as a difference between the two positions: $\mathbf{u}=\mathbf{x}^{\prime}-\mathbf{x}$. Consider two points infinitely close to each other with a defined distance between them. The change in that distance under deformation can be expressed with the following relation:

$$
u_{i k}=\frac{1}{2}\left(\frac{\partial u_{i}}{\partial x_{k}}+\frac{\partial u_{k}}{\partial x_{i}}\right)
$$

Relation (2.34) assumes mathematician-calming small displacement.

Tensor $u_{i k}$ is called deformation tensor and is defined using initial positions of non-displaced points in the crystal. This tensor is symmetric.

For small strains $\mathbf{u}$, the deformation is proportional to stress according to Hooke's law:

$$
\sigma_{i j}=\sum_{k=1}^{3} \sum_{l=1}^{3} c_{i j k l} u_{k l},
$$

where $\sigma_{i j}$ is a stress tensor, and $c_{i j k l}$ is an elastic constants ${ }^{10}$ tensor. Equation (2.35) has summing indexes running from 1 to 3 , therefore in total we have $3^{4}=81$ elastic constants. Basic symmetry relations allow us to lower that number down to 21 . If we further apply translation symmetries based on a type of crystal we have, the amount of non-zero independent elements in elastic constants tensor becomes even less: the higher the symmetry of the lattice the lower it gets.

We can rewrite (2.35) in the following way:

$$
\sigma_{\alpha}=\sum_{\beta=1}^{6} c_{\alpha \beta} u_{\beta},
$$

where I used Voigt notation ${ }^{11}$, applicable to a symmetric tensors of 4 th rank:

$$
\left(\begin{array}{lll}
u_{1} & u_{6} & u_{5} \\
u_{6} & u_{2} & u_{4} \\
u_{5} & u_{4} & u_{3}
\end{array}\right)=\left(\begin{array}{ccc}
u_{11} & 2 u_{12} & 2 u_{13} \\
2 u_{21} & u_{22} & 2 u_{23} \\
2 u_{31} & 2 u_{32} & u_{33}
\end{array}\right)
$$

\subsubsection{Calculating elastic constants using infinitesimal deformations method}

Standard method of performing elastic constants calculation at given volume $V$ uses infinitesimal deformation tensor. The difference in energies

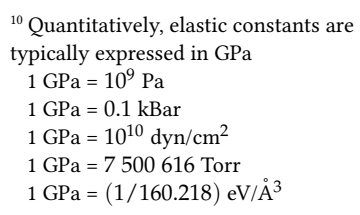

${ }^{11}$ Unless specified otherwise, everywhere else in this chapter this notation is used 
between deformed and non-deformed state as a function of the deformation parameter is assumed to be proportional to a linear combination of secondorder elastic constants. Applied deformation should be isohoric since the effect of volume change is typically more pronounced than the result of application of infinitesimal deformation. Choosing isohoric deformation allows to decouple volume change part so the resulting change in energy is caused solely by infinitesimal deformation. Denoting deformation tensor as $\mathcal{D}(e)$ :

$$
\mathcal{D}(e)=\left(\begin{array}{ccc}
e_{1} & \frac{1}{2} e_{6} & \frac{1}{2} e_{5} \\
\frac{1}{2} e_{6} & e_{2} & \frac{1}{2} e_{4} \\
\frac{1}{2} e_{5} & \frac{1}{2} e_{4} & e_{3}
\end{array}\right)
$$

Energy difference due to applied deformation (2.38) can be expressed as:

$$
E\left(e_{1}, e_{2}, \ldots, e_{6}\right)=E(0)+\frac{1}{2} V \sum_{i=1}^{6} \sum_{j=1}^{6} c_{i j} e_{i} e_{j}+\mathcal{O}\left(e^{3}\right),
$$

where $E(0)$ is the energy of the non-deformed state at volume $V$, and $\mathcal{O}\left(e^{3}\right)$ denotes terms that are proportional to $e^{k}$, for $k \geqslant 3$.

Equation (2.39) allows to get stability criteria for deformed crystal: the energy of the system should be minimal for non-deformed lattice and go up when small deformation present.

A random point in crystal with radius-vector $\mathbf{r}=(x, y, z)$ under deformation (2.38) becomes a point with a new radius-vector $\mathbf{r}^{\prime}=\left(x^{\prime}, y^{\prime}, z^{\prime}\right)$ :

$$
\left(\begin{array}{l}
x^{\prime} \\
y^{\prime} \\
z^{\prime}
\end{array}\right)=(\mathcal{D}(e)+\mathcal{I})\left(\begin{array}{l}
x \\
y \\
z
\end{array}\right)=\left(\begin{array}{l}
\left(1+e_{1}\right) x+\frac{1}{2} e_{6} y+\frac{1}{2} e_{5} z \\
\frac{1}{2} e_{6} x+\left(1+e_{2}\right) y+\frac{1}{2} e_{4} z \\
\frac{1}{2} e_{5} x+\frac{1}{2} e_{4} y+\left(1+e_{3}\right) z
\end{array}\right)
$$

where $\mathcal{I}$ is a $3 \times 3$ entity matrix. In order for $\mathcal{D}(e)$ to be isohoric, we require constraint $\operatorname{det}(\mathcal{D}+\mathcal{I})=1$.

Cubic crystals have only 3 independent non-zero components in the elastic constants tensor, so the change in energy (2.39) under deformation (2.38) is as follows:

$$
\begin{aligned}
\frac{1}{V} \Delta E & =\frac{1}{2} c_{11}\left(e_{1}^{2}+e_{2}^{2}+e_{3}^{2}\right)+c_{12}\left(e_{1} e_{2}+e_{2} e_{3}+e_{1} e_{3}\right) \\
& +\frac{1}{2} c_{44}\left(e_{4}^{2}+e_{5}^{2}+e_{6}^{2}\right)+\mathcal{O}\left(e^{3}\right)
\end{aligned}
$$

In general, if all components of $\mathcal{D}(e)$ are expressed as some variable $\delta$, the difference in energies is linked with elastic constants in the following way:

$$
\Delta E \approx A \cdot V \cdot c \cdot \delta^{2},
$$

where $A$ is some numeric constant, that depends on a deformation applied and $c$ is a corresponding elastic constant or their linear combination. 
For cubic crystals it is common to introduce combinations of elastic constants that presumably have some physical meaning. Our old friend bulk modulus, which is a resistance of the crystal to a uniform compression, can be expressed as:

$$
B=\frac{1}{2}\left(c_{11}+2 c_{12}\right),
$$

and we can introduce shear modulus $c^{\prime}$ :

$$
c^{\prime}=\frac{1}{2}\left(c_{11}-c_{12}\right) \text {. }
$$

To calculate this $c^{\prime}$, we can use tetragonal distortion:

$$
\mathcal{D}_{\mathrm{t}}+\mathcal{I}=\left(\begin{array}{ccc}
1+\delta_{\mathrm{t}} & 0 & 0 \\
0 & 1+\delta_{\mathrm{t}} & 0 \\
0 & 0 & \frac{1}{\left(1+\delta_{\mathrm{t}}\right)^{2}}
\end{array}\right)
$$

which, when substituted in (2.42) leads to the following change in energy:

$$
\Delta E\left(\delta_{\mathrm{t}}\right)=6 V c^{\prime} \delta_{\mathrm{t}}^{2}+\mathcal{O}\left(\delta_{\mathrm{t}}^{3}\right)
$$

However, if we use an orthorhombic distortion:

$$
\mathcal{D}_{\circ}+\mathcal{I}=\left(\begin{array}{ccc}
1+\delta_{\circ} & 0 & 0 \\
0 & 1-\delta_{\circ} & 0 \\
0 & 0 & \frac{1}{1-\delta_{\circ}^{2}}
\end{array}\right),
$$

we get the similar result that is proportional to $c^{\prime}$, but has different numeric prefactor and higher precision:

$$
\Delta E\left(\delta_{\circ}\right)=2 V c^{\prime} \delta_{\circ}^{2}+\mathcal{O}\left(\delta_{\circ}^{4}\right)
$$

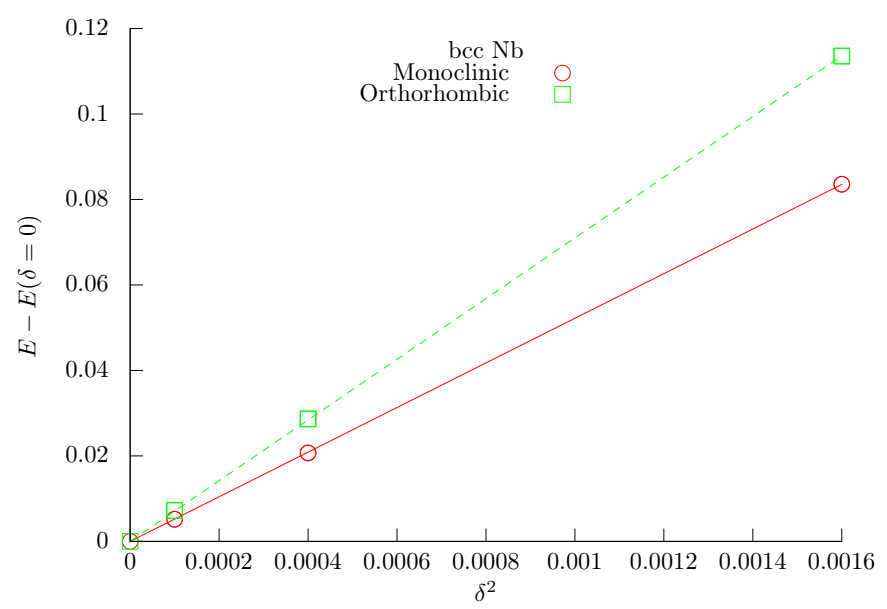

Figure 2.3: Calculated change in energies $\Delta E=E(\delta)-E(\delta=0)$ as a function of squared deformation parameter $\delta^{2}$ in compressed body-centered cubic Niobium with lattice parameter $a=2.8 \AA^{3}$ under monoclinic (green squares) and orthorhombic (red circles) deformation. The slope of a linear fit is proportional to corresponding elastic constant at this volume. 
${ }^{12}$ for symmetric distortion matrices, it is common[96] to take the following 4 points: $\delta=(0.0,0.01,0.02,0.04)$
So it turns out that we can affect precision by choosing proper deformation, without changing the total amount of calculation points ${ }^{12}$.

By analogy, to calculate $c_{44}$, one uses monoclinic distortion:

$$
\mathcal{D}_{\mathrm{m}}+\mathcal{I}=\left(\begin{array}{ccc}
1 & \delta_{\mathrm{m}} & 0 \\
\delta_{\mathrm{m}} & 1 & 0 \\
0 & 0 & \frac{1}{1-\delta_{\mathrm{m}}^{2}}
\end{array}\right),
$$

which, again substituted in (2.42) gives:

$$
\Delta E\left(\delta_{\mathrm{m}}\right)=2 V c_{44} \delta_{\mathrm{m}}^{2}+\mathcal{O}\left(\delta_{\mathrm{m}}^{4}\right)
$$

See Figure 2.3 for example $\Delta E$ calculation.

\section{$2.5 \quad$ Non-linear elasticity}

\footnotetext{
${ }^{13}$ One way of defining significance criterion could be "The displacement becomes comparable with a inter-atomic distance"
}

It is assumed that real crystals have anharmonic inter-atomic interaction potential. If the distortion parameter is significant ${ }^{13}$, the corresponding anharmonic effects become apparent and it is questionable if one can apply infinitesimal elasticity theory. To study such effects we have to include higher order terms when applying elasticity theory

To account for non-linear effects, expression (2.34) becomes a bit more complicated. Corresponding measure of deformation is called Lagrange finite strain tensor:

$$
\eta_{i j}=\frac{1}{2}\left[\left(\frac{\partial u_{i}}{\partial a_{j}}\right)+\left(\frac{\partial u_{j}}{\partial a_{i}}\right)+\left(\frac{\partial u_{k}}{\partial a_{i}} \cdot \frac{\partial u_{k}}{\partial a_{j}}\right)\right],
$$

where $a_{i}$ is an independent variable, and $u_{i}=\left(x_{i}-a_{i}\right)$. Here $a_{i}$ is initial position of a point in crystal before the deformation was applied, and $x_{i}$ is its final position. Using $\eta_{i j}$ and temperature $T$ or entropy $S$ as independent variables, we can introduce various thermodynamic functions such as internal energy $U\left(\eta_{i j}, S\right)$ or Helmholtz's free energy $F\left(\eta_{i j}, T\right)=U-T S$.

Denoting $u_{i j}=\partial u_{i} / \partial a_{j}$ and defining transformation tensor as $\alpha_{i j}=$ $\partial x_{i} / \partial a_{j}$, we can rewrite (2.51) in a more compact form:

$$
\eta_{i j}=\frac{1}{2}\left(\alpha_{i l} \alpha_{l k}-\delta_{i j}\right)
$$

where $\delta_{i j}$ is a Kronecker delta and Einstein summation rule is implied.

\subsubsection{Thermodynamic definition of elastic constants}

Internal energy $U$ and free energy $F$ under applied deformation can be expanded in a series of $\eta$ components:

$$
\begin{aligned}
U\left(\mathbf{R}, \eta_{i j}, S\right)=U(\mathbf{R}, S) & +V \frac{1}{2 !} \sum_{i j k l} C_{i j k l}^{S} \eta_{i j} \eta_{k l}+V \frac{1}{3 !} \sum_{i j k l m n} C_{i j k l m n}^{S} \eta_{i j} \eta_{k l} \eta_{m n} \\
& +V \frac{1}{4 !} \sum_{i j k l m n p q} C_{i j k l m n p q}^{S} \eta_{i j} \eta_{k l} \eta_{m n} \eta_{p q}+\cdots
\end{aligned}
$$




$$
\begin{aligned}
F\left(\mathbf{R}, \eta_{i j}, T\right)=F(\mathbf{R}, T) & +V \frac{1}{2 !} \sum_{i j k l} C_{i j k l}^{T} \eta_{i j} \eta_{k l}+V \frac{1}{3 !} \sum_{i j k l m n} C_{i j k l m n}^{T} \eta_{i j} \eta_{k l} \eta_{m n} \\
& +V \frac{1}{4 !} \sum_{i j k l m n p q} C_{i j k l m n p q}^{T} \eta_{i j} \eta_{k l} \eta_{m n} \eta_{p q}+\cdots
\end{aligned}
$$

Where $C_{i j k l \ldots}^{T}$ and $C_{i j k l \ldots}^{S}$ are isothermic and adiabatic elastic constants. Using these equations (2.53) and (2.54), we introduce elastic constants:

$$
C_{i j k l \ldots}^{T}=\frac{1}{V}\left(\frac{\partial^{n} F}{\partial \eta_{i j} \partial \eta_{k l} \ldots}\right), C_{i j k l \ldots}^{S}=\frac{1}{V}\left(\frac{\partial^{n} U}{\partial \eta_{i j} \partial \eta_{k l} \ldots}\right)
$$

in this expression I assume $n \geqslant 2$

\subsubsection{Effective elastic constants}

For the case of non-zero $T$ and $P$, Helmholtz free energy and internal energy are replaced by Gibbs free energy $G=F+P V$ and Enthalpy $H=U+P V$. Correspondingly, we can easily adapt (2.55) for this case:

$$
\widetilde{C}_{i j k l \ldots}^{T}=\frac{1}{V_{0}}\left(\frac{\partial^{n} G}{\partial \eta_{i j} \partial \eta_{k l} \ldots}\right), \widetilde{C}_{i j k l \ldots}^{S}=\frac{1}{V_{0}}\left(\frac{\partial^{n} H}{\partial \eta_{i j} \partial \eta_{k l} \ldots}\right)
$$

Elastic constants defined this way would also account for the pressure that arises in the system as a counter-reaction to applied deformation $\eta$. Naturally, for the case of $P=0$ definition (2.56) is the same as (2.55). Similarly, for the case of $T=0, F=U-\mathrm{T} \mathcal{S}=U$ and $C_{i j k l \ldots}^{T} \equiv C_{i j k l \ldots}^{S}$.

Effective constants $\widetilde{C}_{i j k l \ldots}$ allow to use the same elastic-constants involving expressions regardless of applied pressure.

\subsubsection{Hydrostatic pressure}

For the case of $P \neq 0$, we define $\Delta G=G(P, T, \eta)-G(P, T, 0), \Delta F=$ $F(P, T, \eta)-F(P, T, 0)$, and $\Delta V=V-V_{0}$. Since $G=F+P V$,

$$
\frac{\Delta G}{V_{0}}=\frac{\Delta F}{V_{0}}+P \frac{\Delta V}{V_{0}}
$$

where $\frac{\Delta V}{V_{0}}=J^{-1}$, and $J=\operatorname{det}\left\|\alpha_{i j}\right\|$ is a Jacobian of transformation.

Gibbs free energy can be also expanded in series of $\eta$ and effective constants:

$$
\begin{aligned}
\frac{\Delta G}{V}=\frac{1}{2 !} \sum_{i j k l} \widetilde{C}_{i j k l} \eta_{i j} \eta_{k l} & +\frac{1}{3 !} \sum_{i j k l m n} \widetilde{C}_{i j k l m n} \eta_{i j} \eta_{k l} \eta_{m n} \\
& +\frac{1}{4 !} \sum_{i j k l m n p q} \widetilde{C}_{i j k l m n p q} \eta_{i j} \eta_{k l} \eta_{m n} \eta_{p q}+\cdots
\end{aligned}
$$

In my work I have not studied elastic constants for the cases of $T \neq 0$. From now on I would omit $S, T$ super-indices to simplify notation. 
where $\Delta G=G(P, T, \eta)-G(P, T, \eta)$

Using (2.52), (2.57), (2.58), and (2.54) we obtain connection between $\alpha_{i j}$, $\eta_{i j}$ and energy expansions up to the fourth order in $\eta_{i j}$ :

$$
\alpha_{i j}=\delta_{i j}+\eta_{i j}-\frac{1}{2} \eta_{k i} \eta_{k j}+\frac{1}{2} \eta_{r k} \eta_{r i} \eta_{k j}-\frac{5}{8} \eta_{k j} \eta_{m k} \eta_{m n} \eta_{n i}
$$

Figure 2.4: bcc $\mathrm{Nb}$ and it's deformed energy with colors representing different ranges of $\eta$ for fitting.

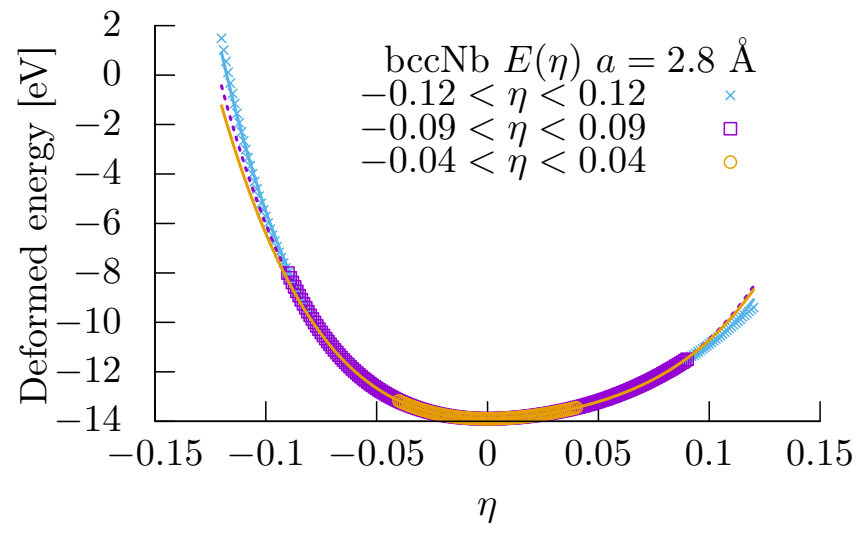

Similar to (2.42) from section 2.4.2, if all non-zero components of $\eta_{i j}$ are expressed via some parameter $\eta$, we can get energy expansion in the series of $\eta$ up to the fourth order of magnitude:

$$
\frac{\Delta U\left(\eta_{i j}\right)}{V_{0}}=v_{1} \eta+v_{2} \eta^{2}+v_{3} \eta^{3}+v_{4} \eta^{4}+o\left(\eta^{4}\right)
$$

where $v_{1}$ would be either 0 or proportional to $P$, and $v_{i}$ for $i \geqslant 2$ represent linear combinations of elastic constants of the corresponding order $i$. The accuracy of the fit would also depend on a range of $\eta$ in question and, in general, depends on a desired set of elastic constants.

Cubic lattice has 3 second-, 6 third-, and 11 fourth-order non-equivalent non-zero elastic constants. In order to find all 11 fourth-order elastic constants, one need to apply 11 nonequivalent deformations that would help resolve the system of linear equations for the fit coefficients in (2.60).

\section{Applications of higher-order elastic constants}

Experimental determination of elastic constants is usually focused on bulk modulus $B$ as a function of pressure, since its the easiest elastic constant to measure [97]. Birch defined a few relations related to second-order elastic 
constants behavior under pressure:

$$
\begin{aligned}
& c_{11}(P)=c_{11}(0)+\phi\left(2 c_{11}+2 c_{12}+6 c_{111}^{*}+4 c_{112}^{*}\right), \\
& c_{12}(P)=c_{12}(0)+\phi\left(-c_{11}-c_{12}+c_{123}^{*}+4 c_{112}^{*}\right), \\
& c_{44}(P)=c_{44}(0)+\phi\left(c_{11}+2 c_{12}+c_{44}+c_{144}^{*} / 2+4 c_{166}^{*}\right),
\end{aligned}
$$

Parameter $\phi$ defines dimensionless compression ratio:

$$
V / V_{0}=(1+2 \phi)^{3 / 2},
$$

where, as before, $V$ is a volume of the system under pressure $P$, and $V_{0}$ is a equilibrium volume.

Elastic constants marked with asterisks in eqs. (2.61), (2.62), and (2.63) are defined by Brugger:

$$
\begin{gathered}
c_{111}^{*}=c_{111} / 6, \\
c_{112}^{*}=c_{112} / 2, \\
c_{123}^{*}=c_{123}, \\
c_{144}^{*}=2 c_{144}, \\
c_{166}^{*}=c_{166} .
\end{gathered}
$$

It should be possible to estimate second-order elastic constants behavior under applied pressure using only zero-pressure measurements of secondand third- order elastic constants.

\subsection{Molecular dynamics}

To quote Richard Feynman, "Everything that living things do can be understood in terms of the jiggling and wiggling of atoms" [98]. In order to simulate this jiggling and wiggling in a computer simulation the motion of interacting particles is simulated using the technique called molecular dynamics ${ }^{14}$. This method treats particles as classical objects with their positions and velocities evolving according to Newton's equations of motion. The following description of Molecular dynamics and corresponding algorithms follows Frenkel and Smit book on molecular simulations [1].

The possibility to compute the equilibrium thermodynamic variables as a statistical average through the means of molecular dynamics is based on the assumption that ergodic hypothesis holds. This hypothesis can be seen as a statement that the ensemble average of a thermodynamic property $A$ is equal to its time average:

$$
\left\langle A\left(\mathbf{R}_{\mathbf{I}}, \mathbf{P}_{\mathbf{I}}\right)\right\rangle=\lim _{t \rightarrow \infty} \frac{1}{t} \int_{0}^{t} A\left(\mathbf{R}_{\mathbf{I}}(\tau), \mathbf{P}_{\mathbf{I}}(\tau)\right) d \tau
$$

There are two distinct classes of molecular dynamics simulations: classical molecular dynamics and ab initio molecular dynamics. Classical molecular dynamics employs classical potential functions that can be based on

\footnotetext{
${ }^{14}$ There are other methods to carry out molecular dynamics simulations based on a combination of classical and $a b$ initio molecular dynamics, and there are other QM/MM methods, such as Monte Carlo, which is quite commonly used to study time evolution of complex systems, but their description is beyond the scope of this book
} 
${ }^{15}$ the other way to run molecular dynamics is Car-Parinello and is often used to study liquids a fit to a more scrutinous quantum-mechanic calculation. This potential is then used to model interaction between particles. Such scheme is usually computationally cheap but lacks transferability compared to ab initio calculations. However, due to relatively low computational cost, classical molecular dynamics allow to study systems with high total count of interacting particles and might not even require a specially designed computer facilities. Generally speaking, classical molecular dynamics cannot describe in a satisfactory way changes in bonding or strong polarization effects, or metal binding. Those effects are usually studied through the means of $a b$ initio methods. Sometimes though, it can be the other way around and classical molecular dynamics simulations can be more accurate than ab initio. For example, van der Waals interaction are long-range in their nature and standard DFT methods such as LDA and GGA cannot describe them accurately, as it was mentioned earlier. Classical mechanic, on the other hand, can give satisfactory results with a simple Lennard-Jones potential:

$$
E_{L J}(R)=4 \varepsilon\left[\left(\frac{\sigma}{R}\right)^{6}+\left(\frac{\sigma}{R}\right)^{12}\right],
$$

with a catch that parameters $\varepsilon$ and $\sigma$ are extracted from experimental data. One can always use more sophisticated classical potentials, but they all require to be fitted to either experimental data or to a result from a more accurate calculation.

In $a b$ initio molecular dynamics (AIMD), forces are derived from electronic structure quantum mechanic calculations of electronic energies. Molecular dynamics implemented in VASP uses Born-Oppenheimer approximation $^{15}$ and the forces are calculated using Hellman-Feynman theorem:

$$
\frac{d E}{d \lambda}=\left\langle\Psi\left|\frac{d \hat{H}}{d \lambda}\right| \Psi\right\rangle
$$

Forces calculated this way are more reliable, but the computation itself is more complicated thus, limiting the typical cell size used in a simulation. Once the forces acting on each atom $\mathbf{f}_{i}$ are found, we can construct Newton's equations of motion:

$$
\begin{aligned}
\frac{d \mathbf{r}_{i}}{d t} & =\frac{\dot{\mathbf{p}}_{i}}{M_{i}} \\
\frac{d \mathbf{p}_{i}}{d t} & =\mathbf{f}_{i}
\end{aligned}
$$

To solve these equations of motion, there exist a few iterative algorithms. One of the commonly used is called velocity Verlet algorithm:

$$
\begin{aligned}
& \mathbf{r}_{i}(t+\Delta t)=\mathbf{r}_{i}(t)+\dot{\mathbf{r}}_{i}(t) \Delta t+\frac{1}{2} \ddot{\mathbf{r}}_{i}(t)(\Delta t)^{2} \\
& \dot{\mathbf{r}}_{i}(t+\Delta t)=\dot{\mathbf{r}}_{i}(t)+\left[\dot{\mathbf{r}}_{i}(t+\Delta t)+\ddot{\mathbf{r}}_{i}(t)\right] \frac{\Delta t}{2},
\end{aligned}
$$

where $\Delta t$ is a time step. When the simulation starts, the forces are calculated using starting positions and DFT calculation, then the (2.74) is used 
to update positions of ions. This advances time of the simulation from $t_{0}$ to $t_{0}+\Delta t$, after which new forces are calculated and process is repeated. Generally speaking, there are no "stop conditions" for molecular dynamics simulation aside from the total amount of timesteps.

\section{Temperature-dependent effective potential method}

In this section I outline the basics of the Temperature-Dependent Effective Potential (TDEP), focusing on the details, relevant to the presentation of the TDEP algorithm in Paper 3. This is by no means original, and the detailed description of the temperature-dependent effective potential method is available in papers by Olle Hellman [42,43].

In order to include vibrational effects, a temperature-dependent model Hamiltonian is introduced:

$$
\hat{H}_{\mathrm{TDEP}}=U_{0}+\frac{\mathbf{p}_{i}^{2}}{2 m_{i}}+\frac{1}{2} \sum_{i j} \mathbf{u}_{i} \overline{\bar{\Phi}}_{i j}(T) \mathbf{u}_{j},
$$

where temperature dependence comes in a form of $\overline{\overline{\boldsymbol{\Phi}}}_{\mathbf{i j}}(T)$, which is a interatomic force constants matrix, and $\mathbf{u}_{i}$ and $\mathbf{p}_{i}$ being the displacement and momentum vectors of the atom $i$.

Inter-atomic force-constants matrix connects the displacement $\mathbf{u}_{i}$ of atom $i$ with the resulting force $\mathbf{f}_{j}$ acting on atom $j$, at temperature $T$. For a system consisting of total $N_{a}$ atoms, in a harmonic approximation the forces and displacements are connected via the force constants as follows:

$$
\left(\begin{array}{c}
\mathbf{f}_{1} \\
\mathbf{f}_{2} \\
\vdots \\
\mathbf{f}_{N_{a}}
\end{array}\right)=\left(\begin{array}{cccc}
\overline{\overline{\boldsymbol{\Phi}}}_{11} & \overline{\overline{\boldsymbol{\Phi}}}_{12} & \ldots & \overline{\overline{\boldsymbol{\Phi}}}_{1 N_{a}} \\
\overline{\overline{\mathbf{\Phi}}}_{21} & \overline{\overline{\boldsymbol{\Phi}}}_{22} & \ldots & \overline{\overline{\boldsymbol{\Phi}}}_{2 N_{a}} \\
\vdots & \vdots & \ddots & \vdots \\
\overline{\overline{\mathbf{\Phi}}}_{N_{a} 1} & \overline{\overline{\mathbf{\Phi}}}_{N_{a} 2} & \ldots & \overline{\overline{\mathbf{\Phi}}}_{N_{a} N_{a}}
\end{array}\right)\left(\begin{array}{c}
\mathbf{u}_{1} \\
\mathbf{u}_{2} \\
\vdots \\
\mathbf{u}_{N_{a}}
\end{array}\right)
$$

TDEP method consists in fitting the parameters $U_{0}$ and $\overline{\overline{\boldsymbol{\Phi}}}_{i j}$ of Hamiltonian in Eq. (2.75) to the results of AIMD simulations at temperature $T$ to give the best possible harmonic approximation of the lattice dynamics of a studied real (anharmonic) system at this temperature. This is achieved via a minimization of the difference in forces calculated from the model Hamiltonian by Eq. (2.76) and reference "real" forces in the studied system.

\subsubsection{Free energy calculations within TDEP method}

In Paper 3, I use TDEP to calculate the $P(V)$ equation of state, that is to determine pressure-volume relations. In a real physical experiment, applied pressure plays the role of the input parameter, but for the simulation in the NVT-ensemble, often employed in AIMD $^{16}$, the pressure is the outcome with the volume being the input parameter instead. In case of NVT-type

\footnotetext{
${ }^{16}$ While it is possible to carry out simulations in the NPT-ensemble, they are currently not well suited for use with TDEP method since the underlying formalism assumes constant volume when fitting results of MD simulation to obtain force-constants matrix in (2.76).
} 
${ }^{17}$ in simulations that require variation of temperature, the inter-atomic force constants can be interpolated as a function of temperature and volume as they often are very smooth in this parameters space. This will increasing the efficiency of the algorithm, by further decreasing the total amount of required AIMD runs. simulation, the pressure is calculated from the free energy of the system in question:

$$
P=-\frac{\partial F}{\partial V}
$$

where $F$ is the Helmholtz free energy, $V$ is the system volume and $P$ is the pressure. Since we are carrying out numerical differentiation, we have to obtain accurate Helmholtz free energy at several volumes to ensure its accurate and smooth behavior. The calculations at each volume require AIMD simulations, making the task numerically demanding and, therefore, it is beneficial for the efficiency of TDEP algorithm to find a way to minimize the total amount of MD runs. It is possible to redistribute terms in calculations of $F$ with TDEP in such a way as to ensure (nearly) linear volume dependence of the terms that require AIMD simulations, while non-linear terms are determined from static calculations. Because of this, the accurate numerical calculations of volume derivatives for a wide range of pressures can be achieved with AIMD executed at just a few volumes.

In the TDEP formalism [42,43], the Helmholtz free energy $F$ consists of two terms, namely vibrational contribution $F_{\mathrm{ph}}$ and a ground state energy $U_{0}$ of an auxiliary model system, given by Hamiltonian (2.75):

$$
F=F_{\mathrm{ph}}+U_{0}
$$

The vibrational contribution $F_{\mathrm{ph}}$ is obtained in the harmonic approximation [99] via

$$
F_{\mathrm{ph}}=\int_{0}^{\infty} g(\omega)\left\{k_{B} T \ln \left[1-\exp \left(-\frac{\hbar \omega}{k_{B} T}\right)\right]+\frac{\hbar \omega}{2}\right\} d \omega,
$$

where $\hbar$ is a reduced Planck's constant, $k_{B}$ is a Boltzmann constant, $\omega$ is the phonon frequency and $g(\omega)$ is the phonon density of states, calculated in a conventional way but with temperature-dependent force constant matrix $\overline{\overline{\boldsymbol{\Phi}}}_{\mathrm{ij}}$ from Eqs. (2.75) and (2.76) ${ }^{17}$. The force constants matrix obtained using TDEP was used to calculate phonon dispersion relations in papers 4 and 5 .

The ground state energy of the model system $U_{0}$ is determined in the following way:

$$
U_{0}=\left\langle U_{\mathrm{MD}}(t)-\frac{1}{2} \sum_{i j \alpha \beta} \Phi_{i j}^{\alpha \beta} u_{i}^{\alpha}(t) u_{j}^{\beta}(t)\right\rangle,
$$

where $t$ is the simulation time, for which the averaging is carried out; $U_{\mathrm{MD}}(t)$ is the temperature-dependent potential energy from AIMD simulation at time $t$; finally, $\alpha$ and $\beta$ are Cartesian coordinates.

I would like to reiterate, that $U_{0}$ is the ground state energy of the auxiliary model system and therefore, typically, differs from both converged potential energy of AIMD simulation and the DFT total energy calculated at $0 \mathrm{~K}$. 
In addition, I would like to note that since the main concept of TDEP is a force-constants matrix elements fitted to an AIMD simulation, it is of utmost importance to ensure that the forces values are converged.

In weakly anharmonic systems around equilibrium $F_{\text {ph }}$ in Eq. (2.78) exhibits almost a linear volume dependence at fixed temperature [100], while the $U_{0}$ term in (2.78) is a non-linear function with respect to volume. To draw advantage from this observation and to reduce the required amount of long AIMD simulations it seems reasonable to rearrange the terms in Eq. (2.78) so that the non-linearity is contained in terms that can be calculated with static DFT calculations, rather than with the tedious $a b$ inito molecular dynamics simulations. To do this, we introduce an auxiliary function $\Delta U$ :

$$
\Delta U=U_{0}-U_{\text {supercell }}^{\text {ideal }}
$$

where $U_{\text {supercell }}^{\text {ideal }}$ is a volume-dependent energy of an "ideal" 18 static supercell, the same that is used in AIMD simulations. Note that the static calculations are carried out at the same temperature as AIMD, and the term includes the electronic entropy. We also define volume-dependent free energy of a corresponding ideal unit cell $U_{\mathrm{uc}}$ that includes the potential energy and the electronic entropy terms at temperature $T$.

In the fortunate case of perfect convergence of all simulations parameters, $U_{\mathrm{uc}} \equiv K \cdot U_{\text {supercell }}^{\text {ideal }}$, where $K$ is an integer number, that accounts for the number of unit cells contained in the super-cell. Unfortunately, the perfect convergence is rarely achieved for any reasonably sized super-cells: fully converged calculations are too resource-consuming. Meanwhile, the calculation of $U_{\mathrm{uc}}(V)$ can be carried out with high accuracy at a dense set of volumes with a relatively low computational costs. This allows to to describe accurately its nonlinear volume dependence. Volume dependence of $\Delta U(V)$ often turns out to be nearly linear, similar to that of $F_{\mathrm{ph}}(V)$. This means that both $\Delta U(V)$ and $F_{\mathrm{ph}}(V)$ require comparatively low amount of calculated data points at different volumes to provide a nice accurate fit and achieve desired accuracy.

Finally, we obtain:

$$
F(V)=F_{\mathrm{ph}}(V)+\Delta U(V)+U_{\mathrm{uc}}(V) .
$$

The non-linear behavior of $F(V)$ is now predominantly concentrated in the volume dependence of the unit-cell $U_{\mathrm{uc}}(V)$. In Paper 3, I consider model system hcp Fe, whose unit-cell consists of two atoms, so the volume dependence of $U_{\mathrm{uc}}(V)$ can be calculated with extremely high precision, since such 2-atom calculations are computationally cheap.

As a result of this arithmetical manipulations, we can effectively increase density of the dataset by an order of magnitude. For example, in
${ }^{18}$ this ideal supercell is "ideal" in a sense that all ions occupy their non-distorted ideal positions, with no displacement whatsoever.

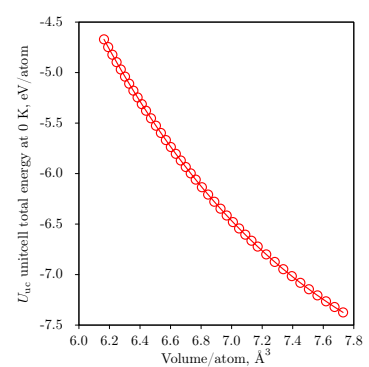

Figure 2.5: $U_{\mathrm{uc}}$ of hcp Fe as a function of volume, calculated at electronic temperature $2000 \mathrm{~K}$ using high-precision settings and a dense set of volumes. This is the main nonlinear term in Eq. (2.81) 
Paper 3 for simulation of hcp Fe at $2000 \mathrm{~K}$ I used only 5 MD-runs within the chosen pressure interval at the temperature in question to fit the nearly linear-dependent terms in (2.82). Once I performed all additional static calculations needed for the use of equation (2.82), including calculations of $U_{\mathrm{uc}}$ at 50 volumes, I effectively have 50 data-points of Helmholtz free energy $F$. Such dataset can be fitted and differentiated with high precision, taking care of any non-linearity.

\subsubsection{Interpolation of force constants matrix components}

${ }^{19}$ strictly speaking, it can be a non-regular temperature dependence, it's just easier to interpolate over a regular grid
Provided that the simulation grid lies on a set of isotherms ${ }^{19}$, it is possible to interpolate components in equation (2.82) to obtain a Helmholtz energy surface $F(T, V)$. This procedure would greatly benefit from an regular grid, though, i.e. it should be a series of calculations on a same set of volumes at different temperatures. Again, preferably grid steps should be kept the same, as it would provide easier fitting in most of the cases, but it is not a strict requirement. This interpolation can be performed in a following manner:

1. Carry out all AIMD simulations on a $T-V$ grid. It would be beneficial to have a grid in a form of a set of points at the same volumes and different temperatures. Having possibility to treat grid-points as a set of isotherms would allow to apply method outlined in section 2.7 .2 , which would increase computational efficiency. I also implicitly assume that at any point inside the convex hull formed by these grid-points, the investigated system is stable and does not undergo any sort of phase transition. Assuming that a set of volumes $\mathcal{V}=\left\{V_{\min }, \ldots V_{\max }\right\}$ with a power $N$, and a set of temperatures $\mathcal{T}=\left\{T_{\min }, \ldots, T_{\max }\right\}$ with a power $M$, the grid $\mathcal{G}$ then would a Cartesian product of those two sets $\mathcal{G}=\mathcal{V} \times \mathcal{T}$ with a power of $N \times M$. Thus, we would have to carry out $N \times M$ AIMD simulations

2. For each of those $N \times M$ simulations, extract force constants matrix components $\overline{\overline{\boldsymbol{\Phi}}}_{\mathbf{i j}}(T)$ using TDEP method. Assuming $N_{\Phi}$ total amount of independent non-zero components in the force constants matrix, this would result in total $N_{\Phi} \times N \times M$ data-points for force constants matrix components.

3. Every independent non-zero element out of $N_{\Phi}$ should be interpolated over its corresponding total $N \times M$ data-points. If the grid is sufficiently dense, a part of it can be set aside during fitting procedure and then be used as a quality test of a resulting fit. This is similar to a crossvalidation technique used widely in statistics. If the dataset is powerful enough, usually this technique is applied multiple times, and each time a new different subset of test points is set aside for validation. Preferably all test subset points should be inside the convex, and far from its 
boundaries, e.g.

$$
\mathcal{G}_{\text {test }}=\left\{(V, T): V_{\min }<V<V_{\max } \text { and } T_{\min }<T<T_{\max }\right\}
$$

Unfortunately, due to resource-consuming nature of AIMD simulations, this quality assurance test is often impossible to carry out since it would leave too few points to provide an accurate fit.

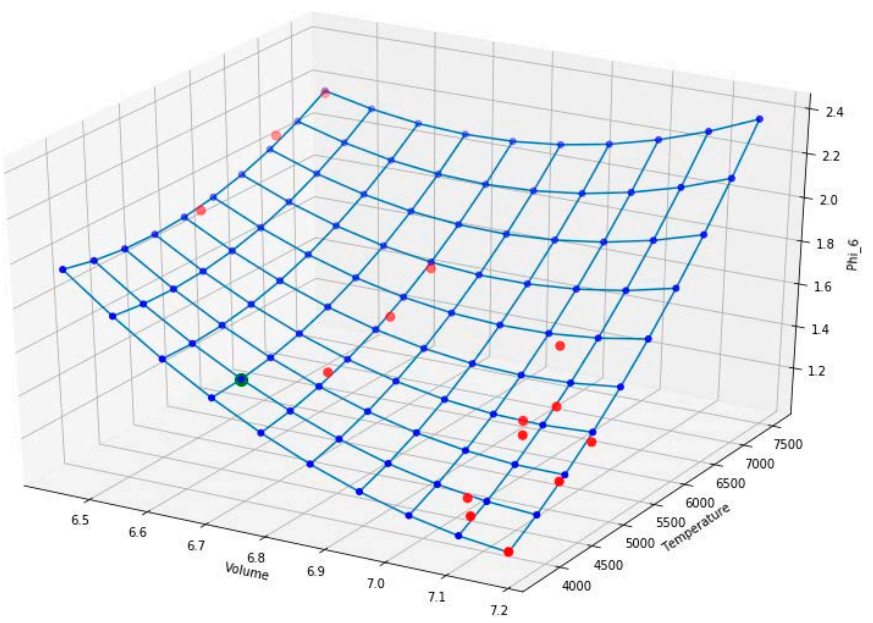

Figure 2.6: Force constants matrix component fitted over the irregular $T-V$ grid. The values extracted from AIMD runs are marked as red dots. The system is hcp Fe. The green dot represents a point outside of convex hull formed by a simulation grid.

4. Each of $N_{\Phi}$ independent non-zero elements in force constants matrix is now a function of both volume and temperature. I can use the resulting force constants matrix $\overline{\overline{\boldsymbol{\Phi}}}_{\mathbf{i j}}(T, V)$ to construct individual components of Eq. (2.82) as a functions of both $V$ and $T$, performing additional calculations if needed, as described above in 2.7.2 and below in section 3.1. This would yield a Helmholtz free energy surface $F(V, T)$ for a subject system in a studied portion of its $V-T$ phase diagram.

5. The Helmholtz free energy surface can be numerically differentiated to obtain pressure $P(T)=-\partial F(V, T) / \partial(V)$. This can be used then to construct Gibbs free energy surface $G(V, T)=F(V, T)+P(T) V$, which provide a valuable insight about system's stability. 


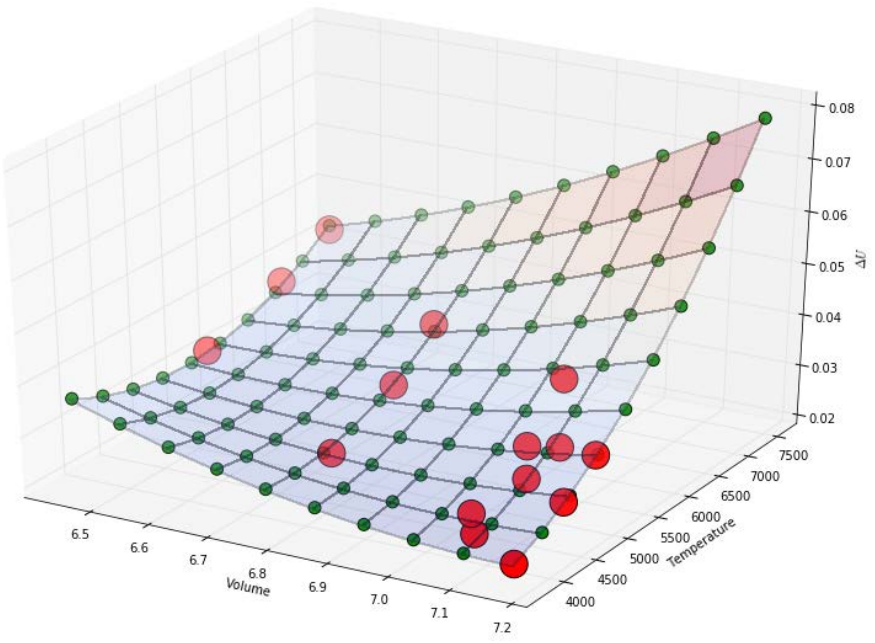

Figure 2.7: $\Delta U$ fit over irregular $T-V$ grid for hcp Fe. Again, while this technically allows to extrapolate outside the convex hull created by the actual AIMD runs (red circles), I consider only interpolation inside this convex hull to be reliable. 


\section{Algorithms}

The results of applying theory outlined in chapter 2 can be summarized as a set of recommendation for the case of high-temperature calculations using TDEP (section 3.1) and higher-order elastic constants calculations (section $3.2)$.

\subsection{Computational algorithm for temperature-dependent effective po-} tential method

The flowchart of the algorithm for the calculation of pressure dependence of the free energy with TDEP method is shown in figure 3.1. Detailed description can be found in paper 3 . The algorithm itself can be outlined as follows:

1. Pick up desired (minimal) number of volumes at which AIMD simulations will be carried out within the range of pressures of interest for the study. Note that the pressure at a finite temperature might differ from the $0 \mathrm{~K}$ equation of state value. Still, calculating $P(V)$ at $0 \mathrm{~K}$ yields a good guess for the initial set of volumes $\left\{V_{i}\right\}$ which can be used for molecular dynamics simulations.

2. For each of the volumes in $\left\{V_{i}\right\}$, carry out low-precision short MD simulation (e.g. with $\Gamma$-point $\mathrm{BZ}$ integration scheme), still long enough for system to to get reasonable estimation of inter-atomic force constants ${ }^{1}$ of the Hamiltonian in eq. (2.75) using TDEP method.

3. Estimate the resulting pressures for each simulation. If the resulting pressures are not close enough to the initial points, adjust the initial set of volumes and repeat the low-precision simulations, until the estimated AIMD pressures are indeed in the range of interest.

4. Using the estimated inter-atomic force constants, it is possible to prepare the super-cells in a thermally excited state following the methodology of West and Estreicher [101] ${ }^{2}$. These super-cells can be used as multiple uncorrelated starting points to carry out highly parallel molecular dynamics simulation. Indeed, the constructed this way starting points are independent of each other and they correspond to physically relevant
${ }^{1}$ for the case of hcp Fe considered in Paper 3 , this takes up to $100-150$ timesteps.

\footnotetext{
${ }^{2}$ in connection with the TDEP method, the realization of this procedure has been described by Steneteg et al. [102]
} 
${ }^{3}$ for simplicity, in figure 3.1 there are only three parallelized blocks, but their amount in reality is only limited by the available resources at hands and can be, for example, an order of magnitude higher

\begin{abstract}
${ }^{4}$ By upsampling I mean a procedure described in, e.g. [103], that allows to carry out highly accurate static electronic structure calculations for the uncorrelated samples, extracted from the low accuracy AIMD instead of using AIMD with fully for the electronic structure calculations. The additional benefit is that this procedure can be done in parallel, since all such simulations are independent of each other.
\end{abstract}

\footnotetext{
${ }^{5}$ the amount of samples needed for the upsampling is determined by the desired accuracy of the simulated property, and needs to be increased until the convergence is obtained. It is common to use stress tensor components and total energy of the system as the target convergence variables.
}

points of the simulated system phase space, virtually eliminating a need for the equilibration of the so designed simulation cells. As a result, the AIMD simulations become highly parallel ${ }^{3}$, allowing efficiently execute TDEP calculations at high-performance massively parallel supercomputers. However, even in a serial execution mode, the procedure described above greatly reduces the time needed to equilibrate the system.

5. Carry out longer converged low-precision simulation to obtain uncorrelated samples for the follow up upsampling ${ }^{4}$. Since two consequent timesteps in molecular dynamics simulation are highly correlated, one in general needs to carry out a simulation with several orders of magnitude more time-steps than the number of samples needed for the upsampling, the static high-precision DFT calculations (dense BZ integration grid, higher cut-off energy).

6. Carry out upsampling. The force constants matrix components then are estimated using these high-precision forces values according to (2.76). The TDEP vibrational contribution $F_{\mathrm{ph}}$ and ground state energy of auxiliary system $U_{0}$ are then need to be calculated according to (2.79) and (2.80), with $U_{\mathrm{MD}}(t)$ term in (2.80) based on the high-precision calculation, and averaging done over the total amount of samples 5

7. Using TDEP, obtain the force constants from high-precision data-points. This gives the phonon part $F_{\mathrm{ph}}$ of Helmholtz free energy as a function of volume as well as the values of the ground state energy $U_{0}$ of our model system. See eqs. (2.79) and (2.80).

8. Calculate $\Delta U$ and $U_{\mathrm{uc}}$ from eq. (2.82) as a functions of volume $V$ in the same range of volumes, with highest possible precision (dense BZ integration grid, high energy cut-off).

9. Combine the calculated dependencies to obtain Helmholtz free energy $F$ in a form of a smooth function of $V$. In paper 3:the data set was dense enough to fit the dependence of $F$ with respect to $V$ with a polynomial of 4 th order and in this way to obtain the analytical expression for the $F(V)$ to be used in the calculation of the equation of state.

10. Calculate the derivative of $F(V)$ to obtain pressure as a function of volume, which yields $P(V)$ equation of state for the simulated system. 


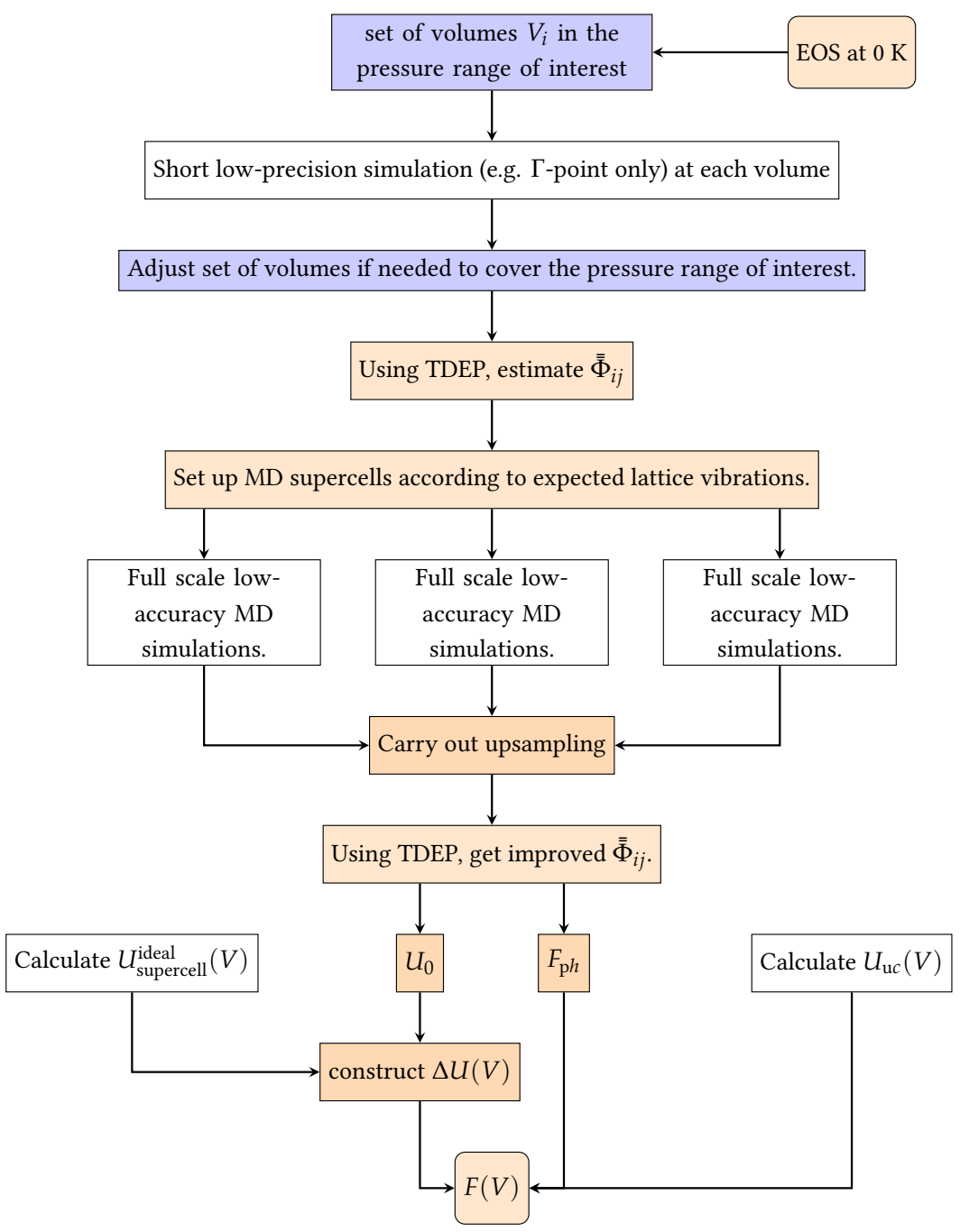

Figure 3.1: Flowchart for Helmholtz free energy calculation algorithm with TDEP method. The step labeled "Full scale lowaccuracy MD simulations" can be done in parallel using different starting super-cells. 


\subsection{Higher-order elastic constants computational algorithm}

Figure 3.2: bcc Mo equation of state. Our calculated data depicted as black triangles. Birch-Murnaghan equation of state fit is show as a red line.
The algorithm is shown graphically in Fig. 3.4. The detailed description can be found in paper 1 . Overall, it can be outlined as follows:

1. Define the set of volumes that will give the pressures in the system in the desired range. This is done by calculating a set of energies for a nondeformed system on a grid of volumes fitted with EOS. An example of such calculation is shown in Fig. 3.2.

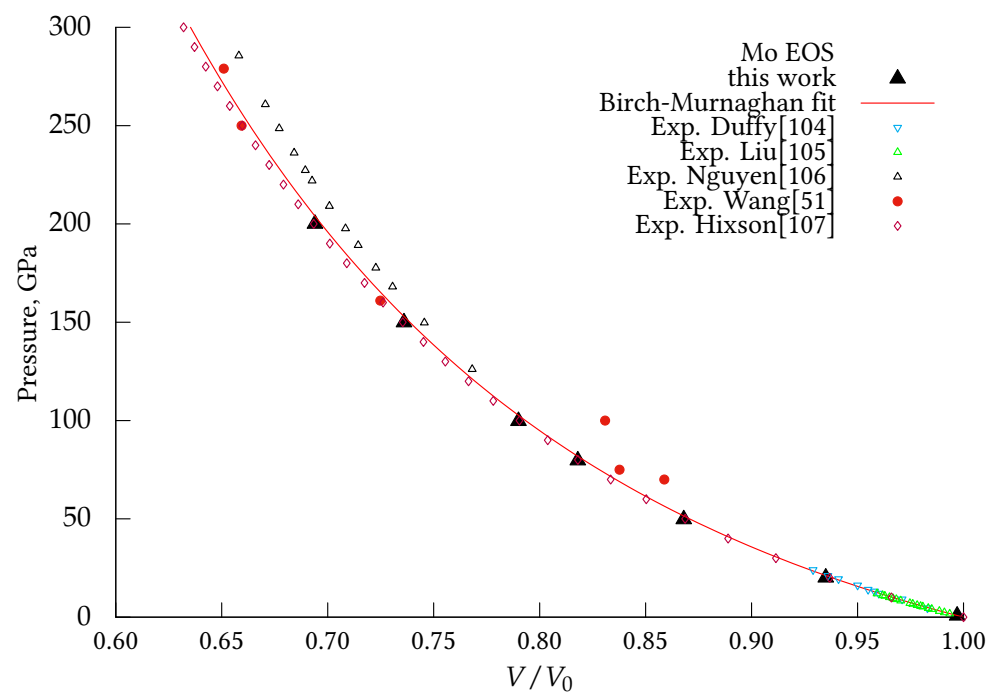

2. Depending on the desired set of elastic constants, decide upon deformation matrices to use. For example, if one's interest consist in finding only the second-order elastic constants as a function of pressure for the system with cubic crystal, three distinct schemes is sufficient.

3. Decide on deformation $\eta$ range, for example $[-0.045 \ldots 0.045]$, and its step value, like 0.003 . This is a matter of balance between smooth $E(\eta)$ dependence and minimization of the total amount of calculations. If TOECs (or FOECs) are of interest, usually the broader range of $\eta$ is required to reach the desired precision.

4. For each of the volumes from step 1 , we calculate $E(\eta)$ dependence for each deformation scheme from step 2 with $\eta$ in a range defined in step 3 . For each of $E(\eta)$ dependencies, we obtain the corresponding coefficients $v_{1,2,3}$ using least-square regression. 
5. First-order coefficients $v_{1}$ can be used to get the equation of state $P(V)$. Knowing those, it is possible to switch from $v_{i}(V)$ to $v_{i}(P)$. For example, if using scheme S1 from Table 3.1, one obtains set of fitting coefficients as a functions of volume: $v_{1}(V), v_{2}(V)$ and $v_{3}(V)$, that can be used to get pressure $P(V)=-v_{1}(V)$, second order elastic constant $\widetilde{C}_{11}(V)=2 v_{2}(V)$ and third order elastic constant $\widetilde{C}_{111}(V)=6 v_{3}(V)$. Each scheme in Table 3.1 would have similar corresponding relations. Combining obtained $P(V)$ with regression coefficients $v_{2,3}(\eta)$ for multiple schemes, we get either direct dependencies or linear combinations of $\widetilde{C}_{i j k}$ as a function of pressure.

Table 3.1: Example of possible deformations and corresponding energy expansions in the series of $\eta$.

\begin{tabular}{c|c|c|c|c}
\multicolumn{2}{c|}{ Deformation scheme } & \multicolumn{3}{|c}{ Deformation energy $v_{1} \eta+v_{2} \eta^{2}+v_{3} \eta^{3}+o\left(\eta^{4}\right)$} \\
\hline $\mathrm{N}$ & $\left(\eta_{1}, \eta_{2}, \eta_{3}, \eta_{4}, \eta_{5}, \eta_{6}\right)$ & $v_{1}$ & $v_{2}$ & $v_{3}$ \\
\hline S1 & $(\eta, 0,0,0,0)$ & $-P$ & $\frac{1}{2} \widetilde{C}_{11}$ & $\frac{1}{6} \widetilde{C}_{111}$ \\
S2 & $(\eta, \eta, 0,0,0)$ & $-2 P$ & $\widetilde{C}_{11}+\widetilde{C}_{12}$ & $\frac{1}{3} \widetilde{C}_{111}+\widetilde{C}_{112}$ \\
S3 & $(\eta,-\eta, 0,0,0)$ & 0 & $\widetilde{C}_{11}-\widetilde{C}_{12}$ & 0 \\
S4 & $(\eta, \eta, \eta, 0,0)$ & $-3 P$ & $32 \widetilde{C}_{11}+3 \widetilde{C}_{12}$ & $\frac{1}{2} \widetilde{C}_{111}+3 \widetilde{C}_{112}+\widetilde{C}_{123}$ \\
S5 & $(0,0,0, \eta, 0,0)$ & 0 & $2 \widetilde{C}_{44}$ & 0 \\
S6 & $(\eta, 0,0, \eta, 0,0)$ & $-P$ & $\frac{1}{2} \widetilde{C}_{11}+2 \widetilde{C}_{44}$ & $\frac{1}{6} \widetilde{C}_{111}+2 \widetilde{C}_{155}$ \\
S7 & $(\eta, 0,0,0,0, \eta)$ & $-P$ & $\frac{1}{2} \widetilde{C}_{11}+2 \widetilde{C}_{44}$ & $\frac{1}{6} \widetilde{C}_{111}+2 \widetilde{C}_{144}$ \\
S8 & $(0,0,0, \eta, \eta, \eta)$ & 0 & $6 \widetilde{C}_{44}$ & $8 \widetilde{C}_{456}$ \\
S9 & $(\eta, 0,0, \eta, \eta, \eta)$ & $-P$ & $\frac{1}{2} \widetilde{C}_{11}+6 \widetilde{C}_{44}$ & $\frac{1}{6} \widetilde{C}_{111}+2 \widetilde{C}_{144}+4 \widetilde{C}_{155}+8 \widetilde{C}_{456}$ \\
\hline
\end{tabular}

If necessary, one needs to adjust the range of $\eta$, to ensure that there are no numerical artifacts. Note that convergence is not always straightforward, as higher range does not guarantee higher precision for any random deformation schemes involved (fig. 3.3). 


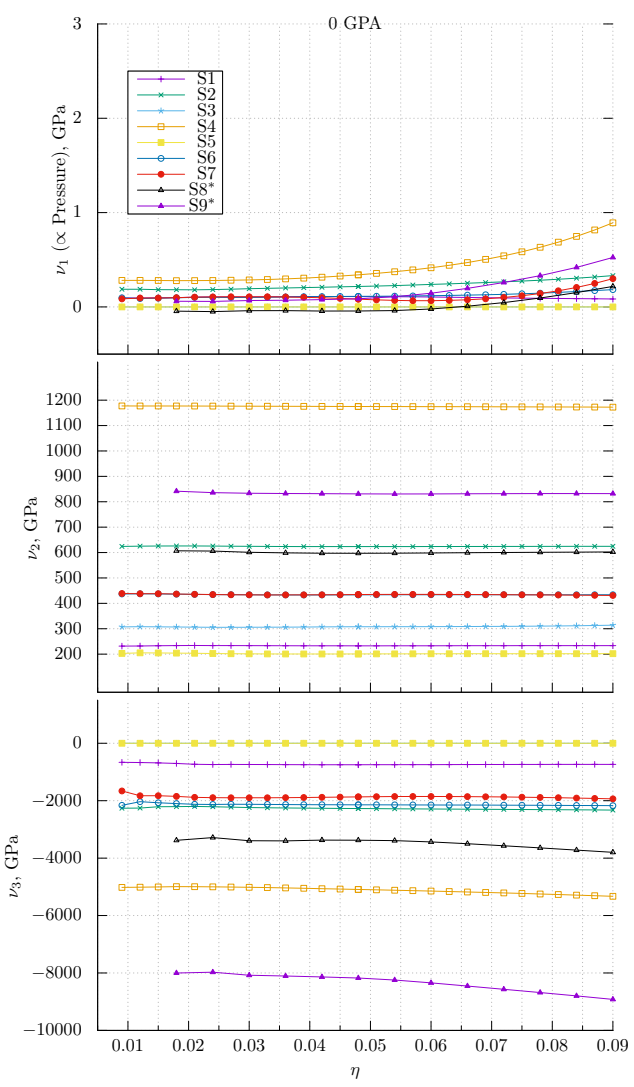

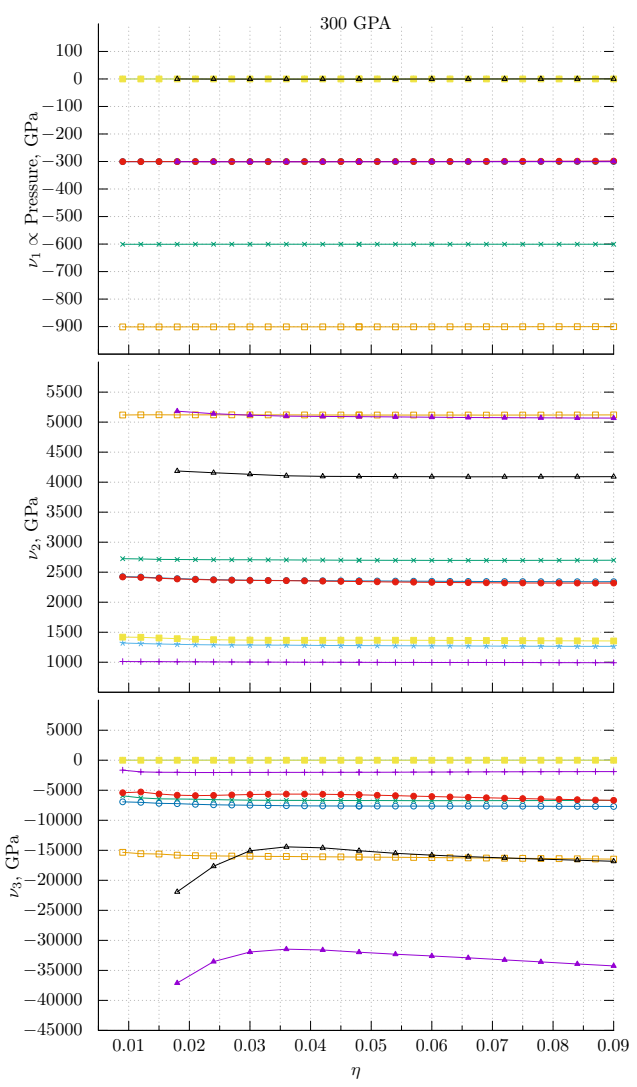

Figure 3.3: Dependency of fit parameters in Schemes from the table on the previous page at $0 \mathrm{GPa}$ (left panels) and $300 \mathrm{GPa}$ (right panels). 


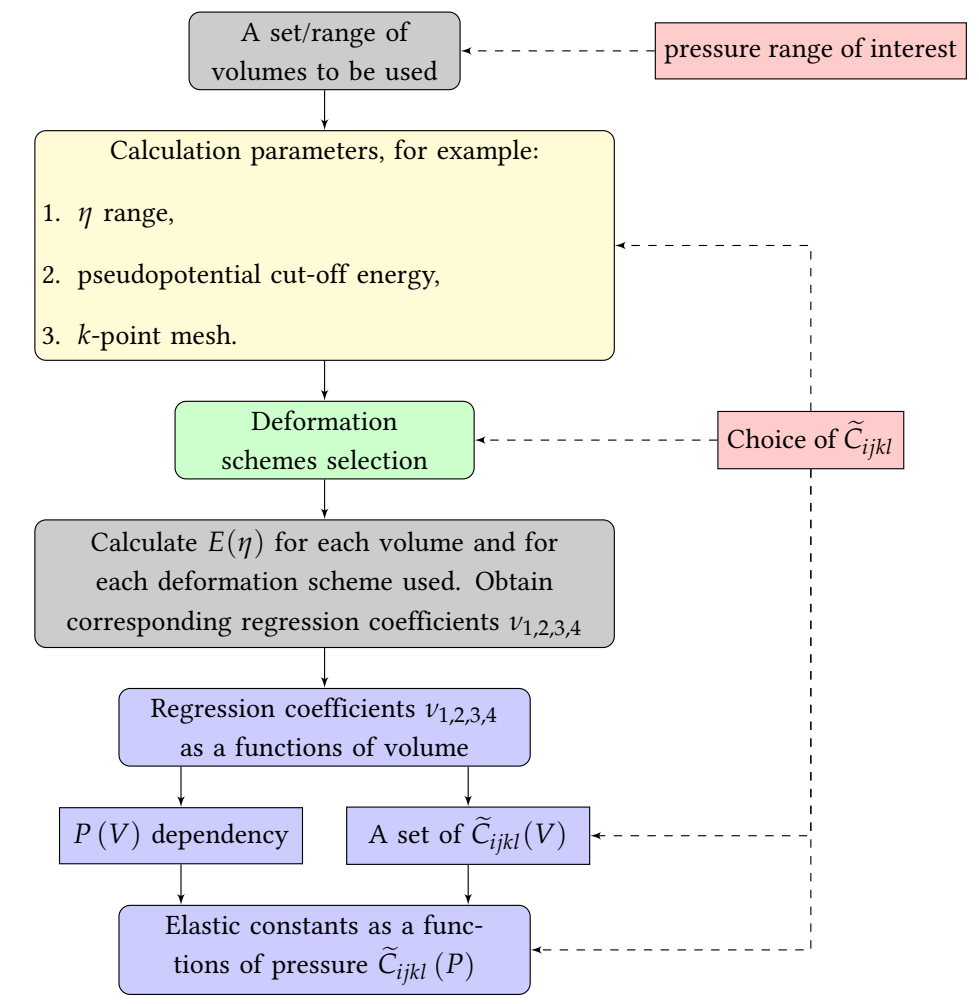

Figure 3.4: Algorithm for calculation of elastic constants as a function of pressure using finite strain tensor. 



\section{Applications for studies of specific ma- terials and properties}

In this chapter I describe the results of application of algorithms from chapter 3 and methods from chapter 2 to the materials described in section 1.2.

\subsection{Equation of state of hexagonal close-packed iron at $2000 \mathrm{~K}$}

The equation of state of hcp Fe at extreme conditions of $\mathrm{T}=2000 \mathrm{~K}$ and $\mathrm{P}=150-$ $450 \mathrm{GPa}$ contains the range of pressures relevant for the geophysical simulations with some leeway. These conditions are within the validity range of the state-of-the-art quasiharmonic approximation (QHA). Therefore, the results of TDEP calculations can be compared directly to those of the quasiharmonic calculations. This comparison is feasible due to the negligible temperature dependence of the force constants of hcp Fe in the validity range of QHA. These results, along with the description of the computational algorithm, are reported in paper 3.

The resulting equation of state is shown in Fig. 4.1 (red circles), alongside with data from QHA calculation by Sha and Cohen [108] (blue line), fitted using Vinet equation of state, eq. (2.32). For the Sha and Cohen data, Vinet equation of state gives equilibrium volume $10.081 \AA$. For our calculation the equilibrium volume was found to be $10.283 \AA$. Note, however, that our data set was limited to high-pressure range where the applicability of GGA-DFT calculations is well established. At lower pressure many-electron effects play important role in simulations of the ground-state properties of hcp Fe $[109,110]$. Therefore, here it is excluded from the consideration.

Very good agreement between two curves in the chosen pressure range shows the reliability of our algorithm, as well as the TDEP method. 


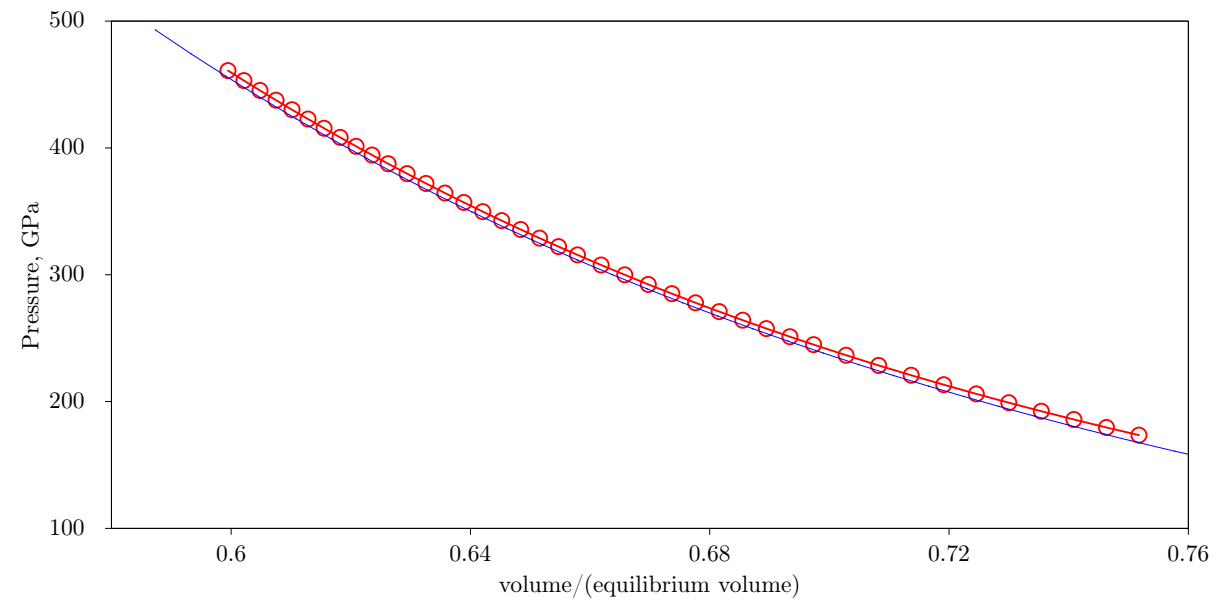

Figure 4.1: Pressure in hcp Fe as a function of $V / V_{0}$ at $T=2000 \mathrm{~K}$. Blue line - data from quasiharmonic calculation by Sha and Cohen[108], red data-points - our work.

4.2.1 Elastic constants of body-centered cubic Molybdenum under pressure

To test the algorithm of the finite strain deformations, we consider a relatively "safe" system of body-centered cubic Molybdenum. Using the described method, second- and third- order elastic constants were calculated in the range of volumes corresponding to pressures from $0 \mathrm{GPa}$ up to $300 \mathrm{GPa}$. These results are reported in paper 1.

Second-order constants of bcc molybdenum, $\widetilde{C}^{\prime}$ and $\widetilde{C}_{44}$ are shown in Figure 4.2. These constants were obtained using distortions from the Table 3.1, namely S3: $(\eta,-\eta, 0,0,0,0)$ and S5: $(0,0,0, \eta, 0,0)$. In the same range of volumes, second-order elastic constants $c^{\prime}$ and $c_{44}$ were also calculated using standard infinitesimal deformations method.

The perfect agreement between the pressure dependencies of $c_{44}$ obtained by standard method and $\widetilde{C}_{44}$ obtained by finite strains method is observed in the bottom panel of Fig. 4.2. Top panel of Fig. 4.2 features difference between the values of $c^{\prime}$ provided with standard infinitesimal method and $\widetilde{C}^{\prime}$ from finite deformations technique. The difference is around $3 \mathrm{GPa}$ between those two values is observed at pressure around $100 \mathrm{GPa}$. The difference goes up with pressure up to $14 \mathrm{GPa}$ at $300 \mathrm{GPa}$. Overal, the agreement is satifactory between $c_{44}(P)$ and $\widetilde{C}_{44}(P)$ obtained by two different method. The difference in pressure-dependency of $\widetilde{C}_{44}(P)$ compared to a less linear $\widetilde{C}^{\prime}(P)$ was observed previously [111]. The difference between $\widetilde{C}^{\prime}$ and $c^{\prime}$ from the standard method might be due to higher-order contributions by corresponding distortions $e_{i j}$, implicitly included at higher 
pressures in values obtained using infinitesimal strains.

Discussing the absolute values of the SOECs at $0 \mathrm{GPa}$, our results are quite consistent with the literature data, from both experimental [112-114] and theoretical [115] sources. The difference between the calculated and experimentally obtained values does not exceed $1 \%$ for $c_{11}$ : our calculations yield $465.9 \mathrm{GPa}$, while [112] gives $465 \pm 0.6$ and [113] provides $461.7 \pm 7.9$ $\mathrm{GPa}$. The obtained value of $c_{12}$ at zero pressure also shows good agreement with experiment: 163 in out work, $164.7 \pm 9.9$ in [113], and $163 \pm 2$ in [112]. The $c_{44}$ is slightly underestimated in comparison with experiment, which is well known issue [115] for PAW calculations: our calculations provide 100 $\mathrm{GPa}$, while $108.4 \pm 3.1$ is given in [113], and $109.09 \pm 0.9$ in [112]. Taking into account the experimental error, these results are rather reasonable. Obtained bulk modulus value $(260.5 \mathrm{GPa})$, is in excellent agreement with the experiment (261.8 $\mathrm{GPa}$ and $269 \pm 9 \mathrm{GPa}$ in [113] and [114] respectively).

Third-order elastic constants of body-centered Molybdenum, calculated using finite strains method are shown in Figure 4.3. In order to calculate them, all 9 deformations from the Table 3.1 were employed. While there are total 7 deformations in the table 3.1 that have non-zero third-order term, there are only 6 third-order elastic constants for bcc lattice. Third-order elastic constant $\widetilde{C}_{456}$ was obtained by averaging from the values of $v_{3}(V)$ obtained from $(0,0,0, \eta, \eta, \eta)$ and $(\eta, 0,0, \eta, \eta, \eta)$ distortions. This was done partially to compensate for bad convergence of $v_{3}(V)$ in terms of $\eta$ range.

Left panel of Figure 4.3 depicts $\widetilde{C}_{111}$, which is roughly 8 times bigger than any other third order constant of body-centered cubic Molybdenum. The right panel depicts all other third-order elastic constants, in particular
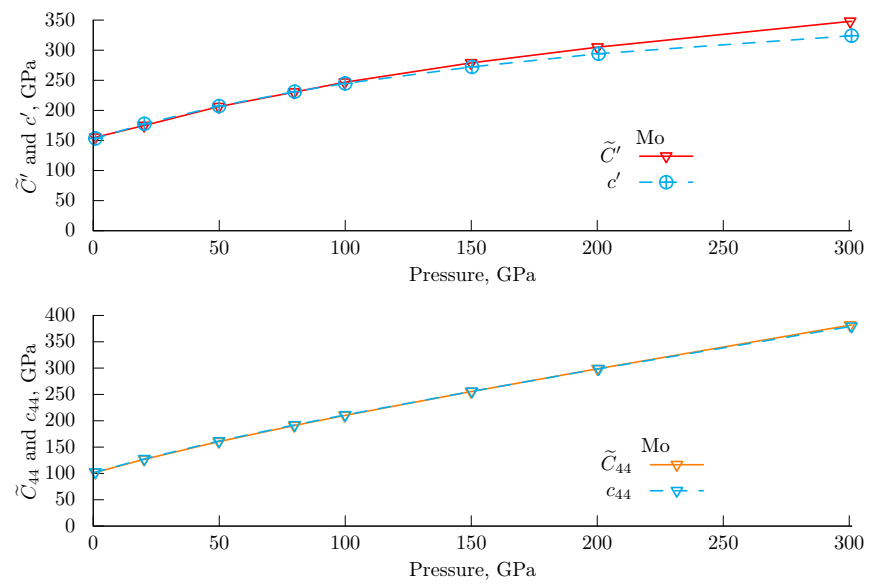

Figure 4.2: $C_{44}$ and $C^{\prime}$ in bcc Molybdenum as a function of pressure, calculated using finite strain method and standard infinitesimal technique. 

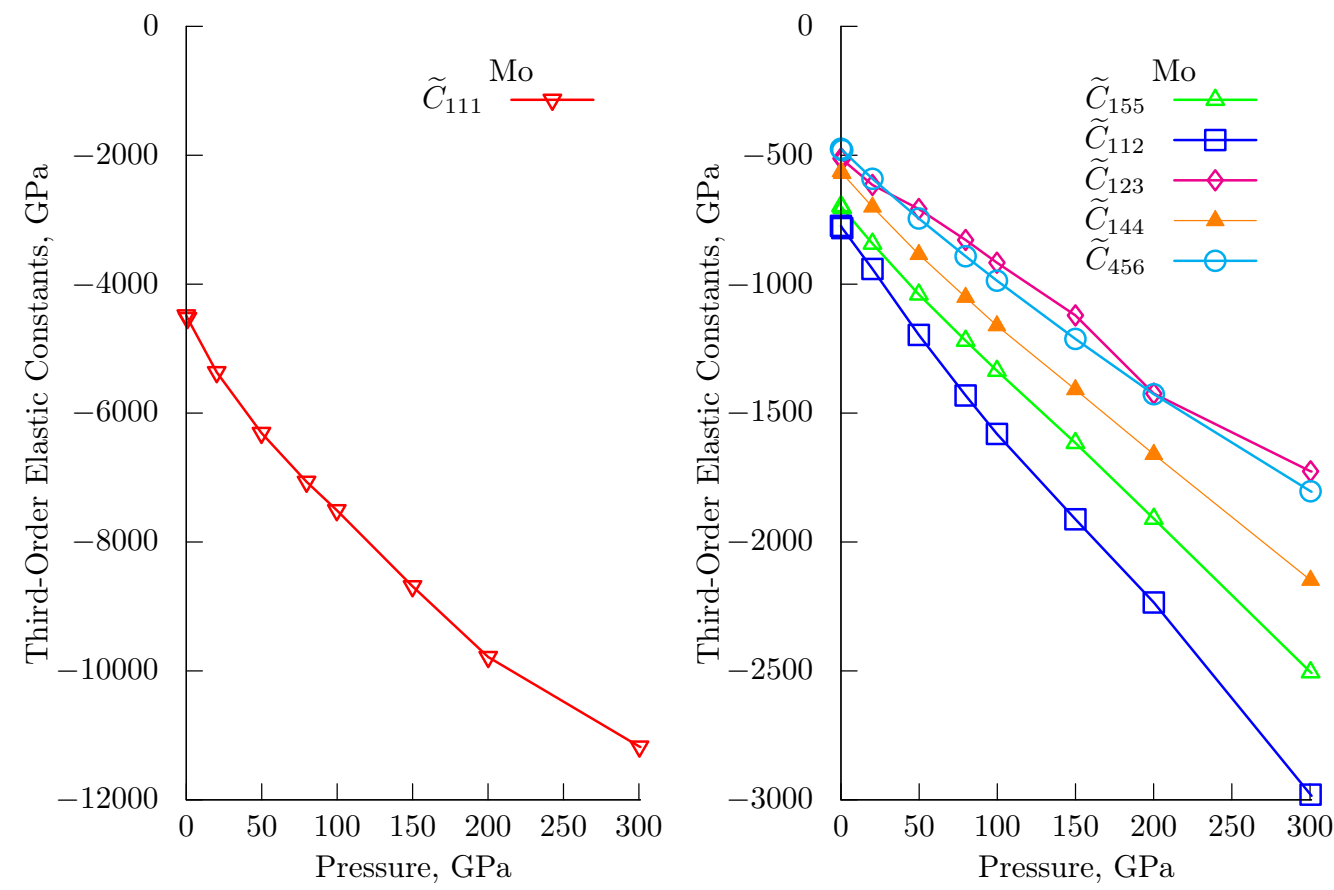

Figure 4.3: Full set of third-order elastic constants of bcc Molybdenum as a function of pressure.

$\widetilde{C}_{155}$ (green contoured triangles), $\widetilde{C}_{112}$ (blue squares), $\widetilde{C}_{123}$ (pink diamonds), $\widetilde{C}_{144}$ (orange filled triangles) and $\widetilde{C}_{456}$ (blue circles). The points are connected as a guide for an eye.

Molybdenum exhibits no peculiarities of elastic behavior at pressure up to $300 \mathrm{GPa}$, with the exception of slight softening in of $\widetilde{C}^{\prime}$ at higher pressures (see Fig. 4.2). At the same time there is no evidence of peculiarities in the behavior of $\widetilde{C}_{44}$ in the same pressure range.

\subsubsection{Elastic constants of body-centered cubic Tantalum under pressure}

Finite small deformation method was applied to body-centered cubic Tantalum to investigate its elastic constants under pressure. These calculations were carried out with cut off energy of $500 \mathrm{eV}$ and $k$-points mesh of $30 \times 30 \times 30$ using PBE-GGA PAW-potential with 11 electrons in a valence band.

Figure 4.4 shows static constants of bcc Tantalum as a function of pressure. Our data from finite deformations method is compared to results of LAPW calculation by Gülseren and Cohen [116], in which they use in- 


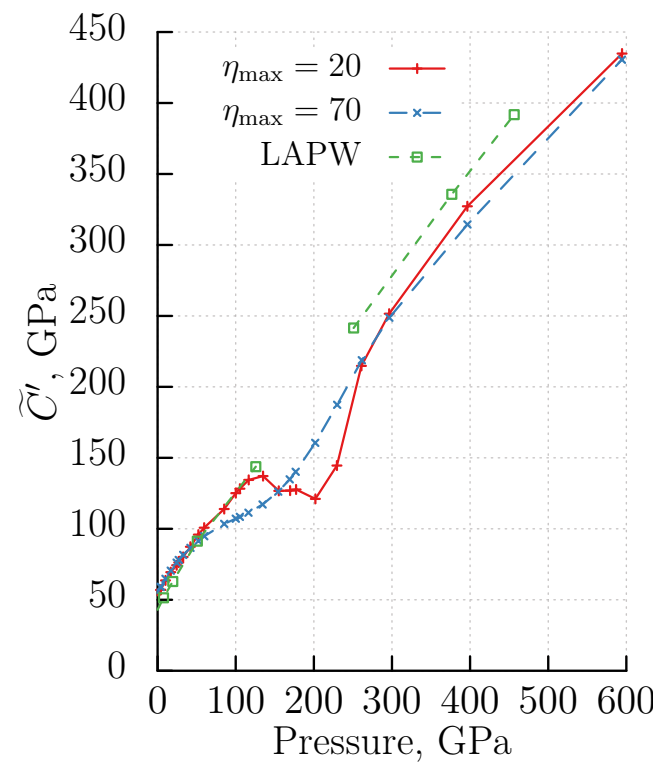

finitesimal deformation. Tantalum in its body-centered cubic structure is known to be a high-pressure standard which stable up to $174 \mathrm{GPa}$ when compressed in diamond anvil cell [117]. Stability conditions are fulfilled in the whole pressure range studied. There's a slight softening in the range of $150-200 \mathrm{GPa}$, that other studies also notice $[116,118]$, and propose that it is caused by electronic topological transition which is occuring due to Fermi surface reconfiguration.

Note that the behaviour of the $\widetilde{C}^{\prime}(P)$ curve is drastically affected by the maximum deformation $\eta_{\max }$, with noticeable sagging at $\eta_{\max }=20$, that gets a lift when a higher deformation range is considered. The study [116] omits the values for $C_{11}$ and $C_{12}$ in the said range. Furthermore, left panel of the Figure 4.5 shows third-order elastic constant $c_{111}$ of bcc Tantalum as a function of pressure at two different $\eta_{\max }$ values. Note the similar sagging around $150-200 \mathrm{GPa}$ for $\eta_{\max }=20$ as it is for second-order elastic constant $\widetilde{C}^{\prime}$. The behaviour with $\eta_{\max }=70$ seems to provide less numerical artifacts and therefore is considered to be a more reasonable setting. It is used for calculation of all other third-order elastic constants, featured on the right panel of Figure 4.5 .

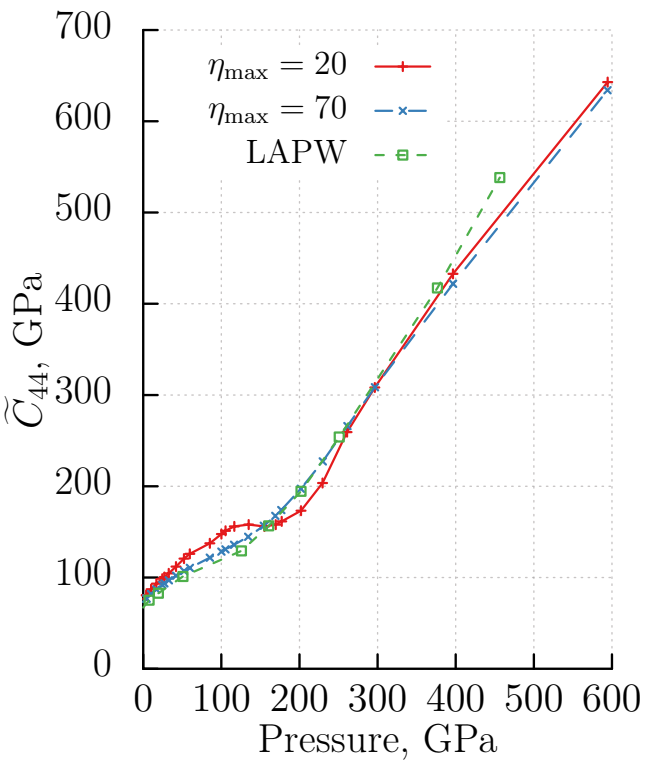

Figure 4.4: $C_{44}$ and $C^{\prime}$ in bcc Tantalum as a function of pressure for two values of $\eta_{\max }$. Green squares connected with dashed line is a LAPW calculation by Gülseren and Cohen [116]. 

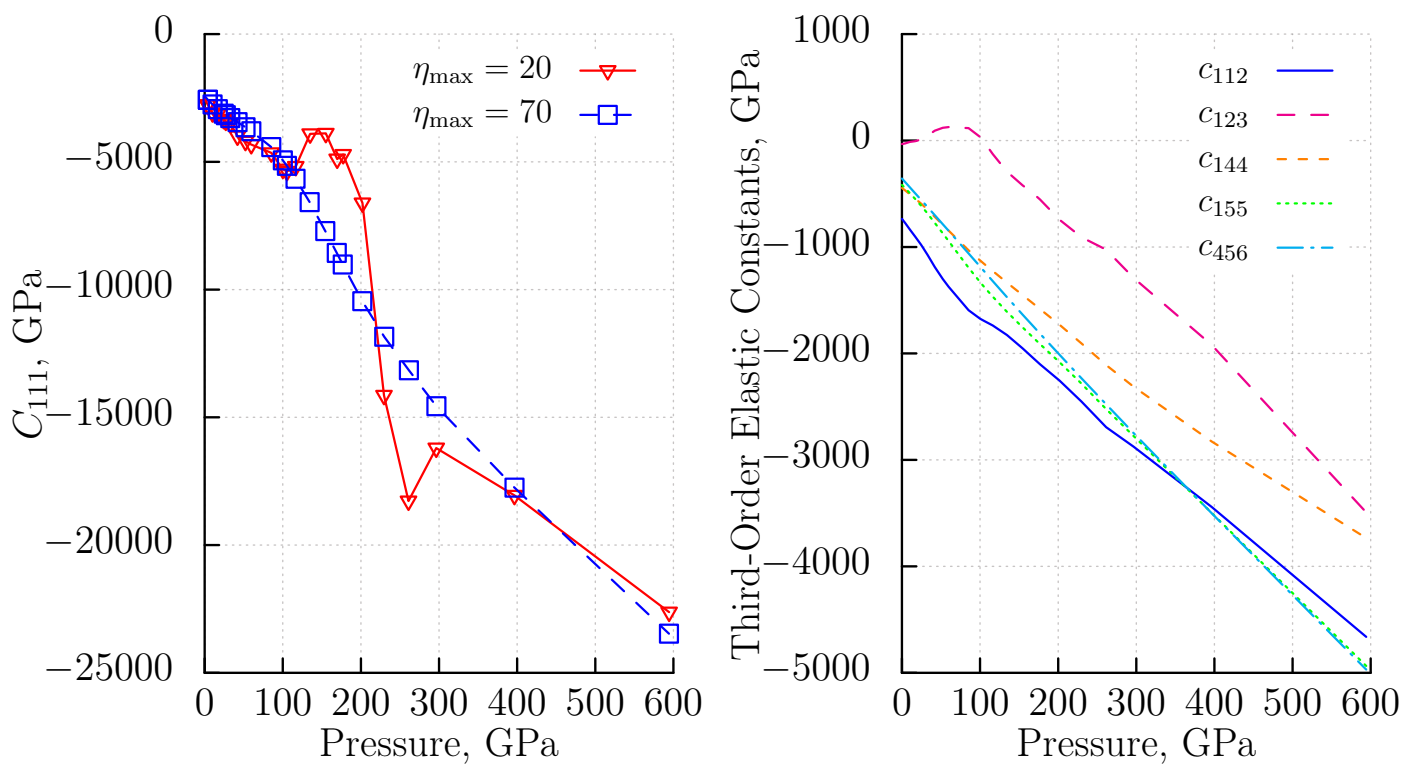

Figure 4.5: Third order elastic constants of bcc Tantalum as a function of pressure 


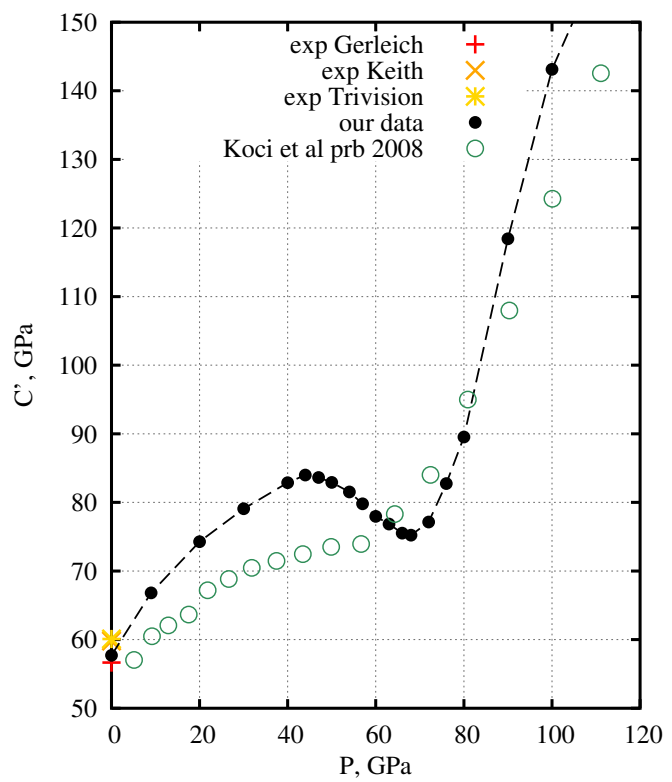

\section{Elastic constants of body-centered cubic Niobium under pressure}

Figure 4.6 shows second-order elastic constants in body-centered cubic Niobium, calculated using finite deformations methods in a range of pressures from 0 to $120 \mathrm{GPa}$, compared to available literature data. Note how all calculated methods underestimate the value of $C_{44}$ shear modulus compared to its experimental value. Results reported here were carried out with cutoff energy of $800 \mathrm{eV}$ and $k$-points mesh of $34 \times 34 \times 34$ using PBE-GGA PAW-potential with 13 electrons in a valence band.

Figure 4.9 shows the dependency of $\Delta E\left(\delta_{m}\right)$ and $\sigma\left(\delta_{m}\right)$ at three selected pressures of $0 \mathrm{GPa}, 68 \mathrm{GPa}$ and $120 \mathrm{GPa}$. The three panes in the right column of Figure 4.9 essentially represent a Hooke's law at these pressures. It is noticeable that, while non-linear stress-strain behaviour around $\delta=0$ in Niobium is low at $0 \mathrm{GPa}$, it is more pronounced at $68 \mathrm{GPa}$ and a regular linear fit through this data set is not enough to describe this behaviour. These pictures allow to conclude that elastic response of Niobium under conditions of $T=0 \mathrm{~K}$ and pressures in the range $0-120 \mathrm{GPa}$ limits the application range of the Hooke's law.

This observation alone does not justify any sort of electronic topological transition, but rather hints at its possibility. To investigate it further, it is important to study band structure of the said material and its behaviour both under pressure and under applied deformation. Figure 4.7 shows band structure of bcc Niobium calculated at $P=0 \mathrm{GPa}$ and $T=0 \mathrm{~K}$. There is an 
${ }^{1}$ rather, Fermi level would change since $E_{F}(P>0)>E_{F}(P=0)$

Figure 4.7: (left)The band structure of bcc Niobium at $0 \mathrm{GPa}$ and $T=0 \mathrm{~K}$. Symmetry point $\Gamma_{25}^{\prime}$ is above Fermi level.

Figure 4.8: The band structure of bcc Niobium at $90 \mathrm{GPa}$ and $\mathrm{T}=0 \mathrm{~K}$; no applied deformation. Symmetry point $\Gamma_{25}^{\prime}$ is at Fermi level. interesting point above Fermi level, marked as $\Gamma_{25}^{\prime}$ where triple degeneracy is observed. It is reasonable to assume that with increased compression the band structure would change and this point would move with respect to Fermi level ${ }^{1}$. We estimate this pressure to be around $90 \mathrm{GPa}$, see Figure 4.8.

However, even at lower pressures applied deformation would change the band structure of bcc Niobium in this particular region. The close examination of a band structure of Niobium at $68 \mathrm{GPa}$ suggests an electronic topological transition due to applied deformation, see Figure 4.10.
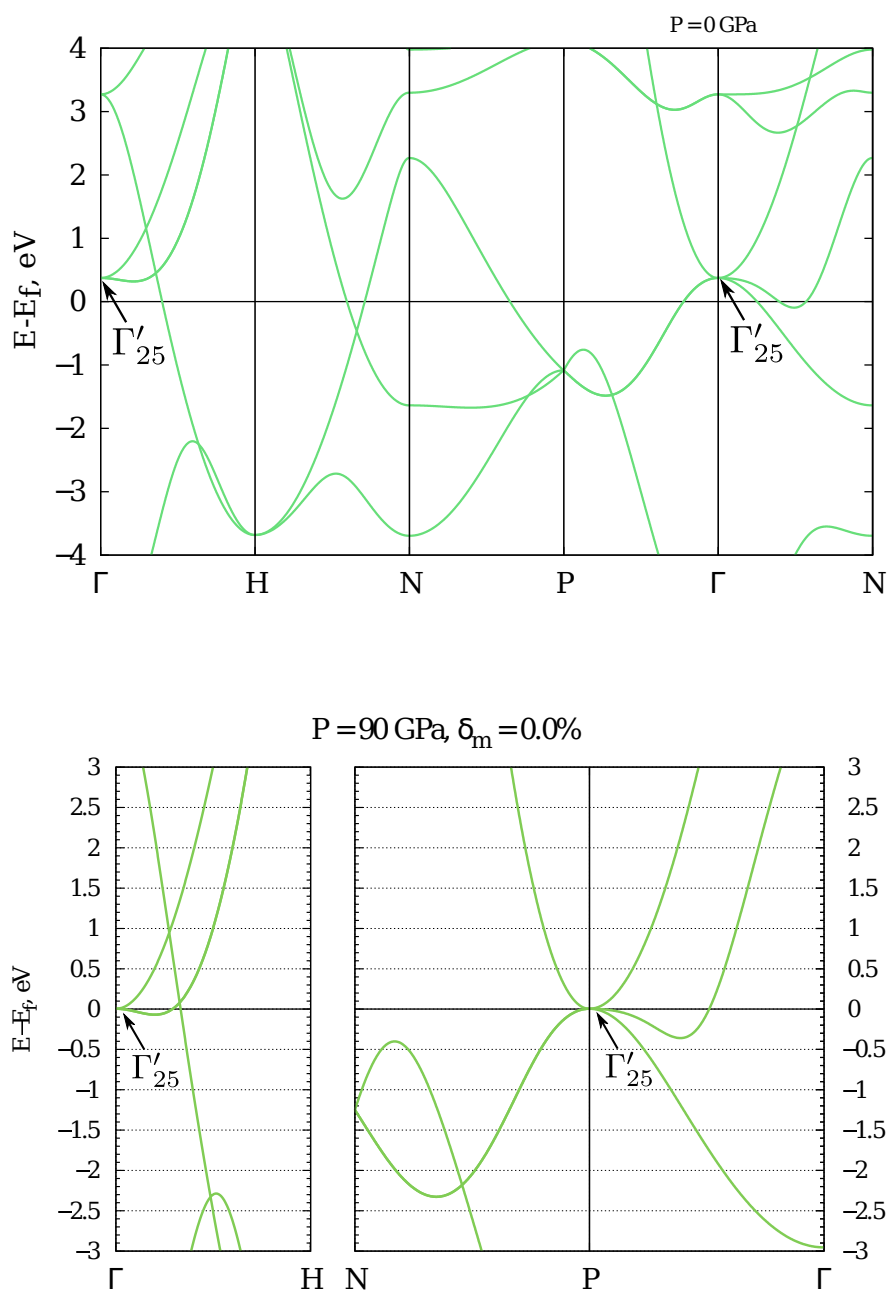
Figure 4.9: Calculated dependencies $\Delta E\left(\delta_{m}\right)$ (left column), and $\sigma\left(\delta_{m}\right)$ (right column). Symbols $*$ depict the results of our calculation, with green line representing a linear fit through it. The blue line shows corresponding polynomial fit.
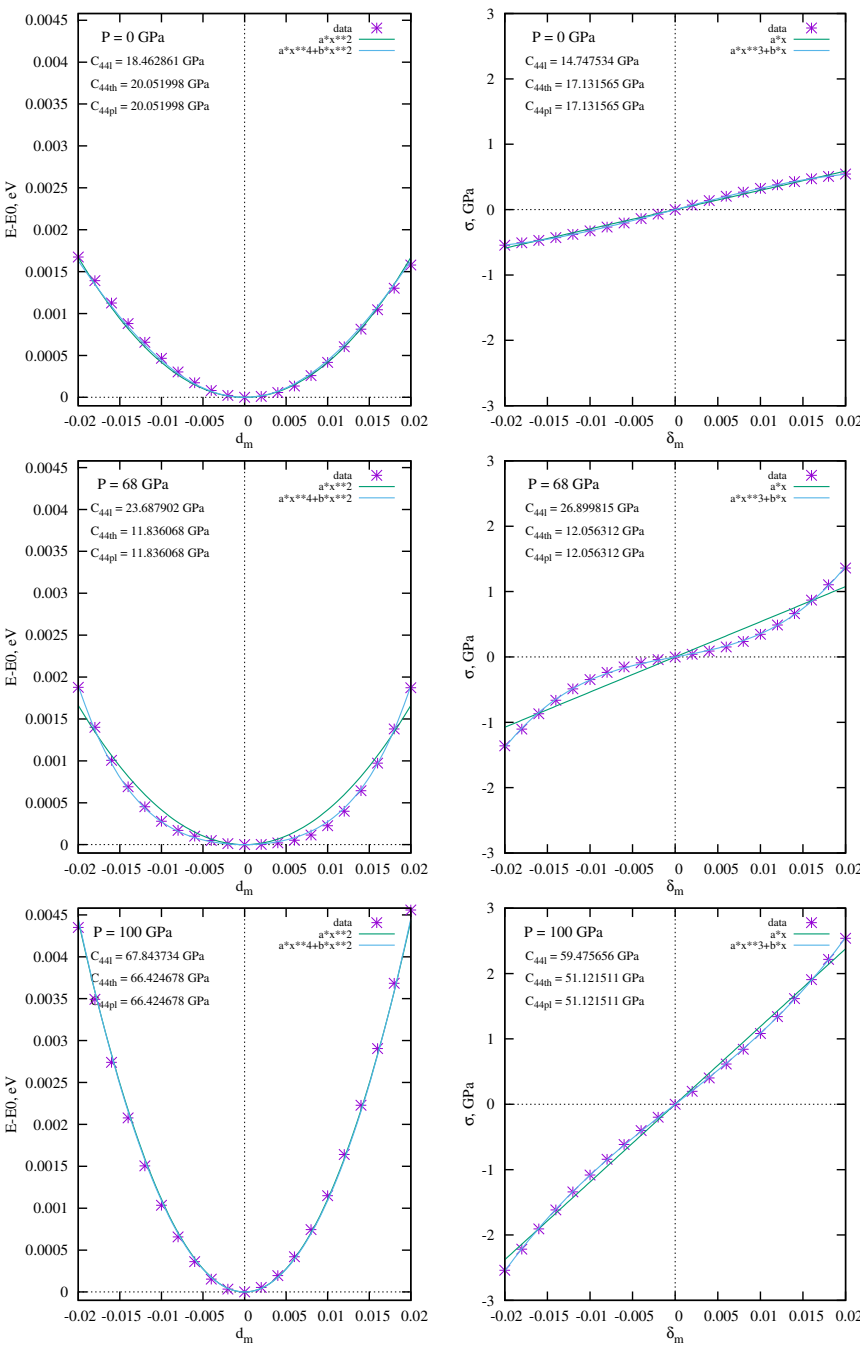
Figure 4.10: Monoclinic deformation effect on a Niobium band structure at $\mathrm{P}=68 \mathrm{GPa}$ and $\mathrm{T}=0 \mathrm{~K}$. Symmetry point $\Gamma_{25}^{\prime}$ is above Fermi level when there is no deformation.
${ }^{2}$ Six total corrections were used: 4 energy corrections, namely $\mathrm{PBE}+\mathrm{D} 3, \mathrm{PBE}+\mathrm{D} 3+\mathrm{BJ}$, $\mathrm{PBE}+\mathrm{TS}, \mathrm{PBE}+\mathrm{TS}+\mathrm{SCS}$, and two non-local functionals: optB86b and rev-vdW-DF2

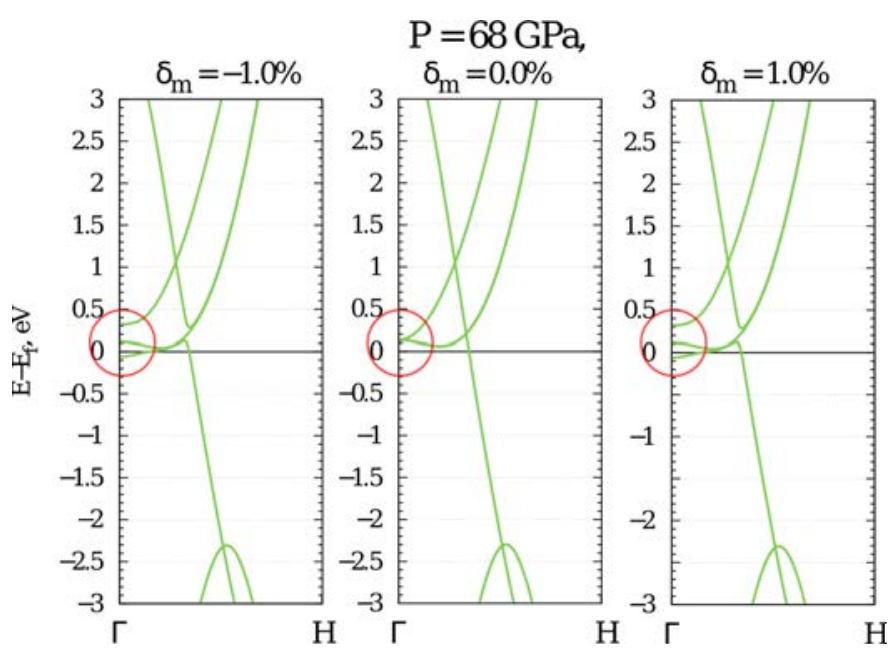

\section{Effect of van der Waals corrections on the description of graphite}

and diamond

The commonly used van der Waals corrections ${ }^{2}$ were applied to investigate graphite and diamond structural parameters under pressure and equilibrium elastic constants. The unit-cells were compressed up to the volumes corresponding to roughly $30 \mathrm{GPa}$. We also suggest a set of metrics to estimate quality of the used approximations. The elastic constants were derived from stress-strain relationship [123,124]. These results are reported in paper 2.

In case of graphite, there are two parameters in its hexagonal lattice: in-planar distance $a$ and inter-planar distance $c$. When studying hexagonal structures, it is common to introduce their ratio, called $c / a$-ratio. Diamond structure, on the other hand, has only one independent lattice parameter $a$.

Convergence tests found that a cutoff energy of $1000 \mathrm{eV}$ and $23 \times 23 \times$ $11 \mathrm{k}$-point grids are sufficient to describe AB-stacked graphite. Structural optimizations were performed using the conjugate-gradient algorithm until the forces were smaller than $1 \mathrm{meV} / \AA$. The diamond structure was converged using a $9 \times 9 \times 9 k$-point mesh.

\section{Bulk properties}

Figure 4.11 shows Graphite structural parameters as a function of pressure and comparison of it with the experimental data. Overall, all tested vdW corrections exhibit the same general pressure dependence behavior of the $c$ lattice parameter of graphite, that is, a reduction by approximately $1.5 \AA$ when increasing the pressure from $0 \mathrm{GPa}$ to $30 \mathrm{GPa}$. LDA underestimates 
the $c$ lattice constant, with a difference of $1-2 \%$ compared to the experimental values of Wang et al.[125] over a wide pressure range. $\mathrm{PBE}+\mathrm{D} 3+\mathrm{BJ}$ gives the best performance across the whole pressure range, although both nonlocal functionals and PBE+TS+SCS also behave well. ${ }^{3}$ The PBE+TS functional has a more complex pressure-dependence than the other functionals, with a maximum error of approximately $2 \%$ occurring at approximately $7 \mathrm{GPa}$, before reducing at higher pressures. The PBE+D3 functional, while significantly overestimating $c_{0}$, performs better at higher pressures. As for at $0 \mathrm{GPa}$, all of the considered functionals reproduce the in-plane lattice constant with an error of less than $0.7 \%$ across the entire pressure range. The best performance is achieved by the rev-vdW-DF2 and PBE+TS+SCS functionals.

In order to determine which functional gives the best agreement with experiment over a variety of different descriptors such as lattice constants and bulk modulus, we suggest a MARE metric that is defined as the mean absolute error achieved by a functional as:

$$
M A R E=\frac{100}{n} \sum_{t=1}^{n}\left|\frac{V_{\text {exp }}-V_{\text {theo }}}{V_{\text {exp }}}\right|
$$

where $n$ is the number of descriptors being considered, $V_{\text {exp }}$ is the experimental value and $V_{\text {theo }}$ is the theoretical value. MARE is inversely proportional to accuracy: lower MARE means higher agreement with the experimental data. Graphite ground state properties are reported in table 4.1. All of the vdW-inclusive calculations give an improvement over LDA of the inplane lattice constant, $a_{0}$. Whereas LDA gives an error of $0.6 \%$, each of the vdW functionals give errors of less than $0.2 \%$. The best performance across all descriptors is given by $\mathrm{PBE}+\mathrm{TS}+\mathrm{SCS}$, with a MARE value of $0.1 \%$. This is followed by the optB86b functional (2.3\%) and then the PBE+D3+BJ (4.2\%) functional. The worst performing functional is $\mathrm{PBE}+\mathrm{D} 3$ with a MARE value of $7.7 \%$.

Diamond ground state properties are reported in table 4.2. Similar to graphite, all functionals perform well in the description of the bulk lattice constant, with a maximum error of less than $0.9 \%$ occurring with the LDA functional. All of the dispersion-corrected functionals improve slightly on this, with errors reducing to no more than $0.4 \%$. The dispersion-corrected functionals are also capable of improving the description of the bulk modulus compared to LDA, with the four $\mathrm{C}_{6}$-corrected functionals reducing the error from $5.4 \%$ to less than $0.7 \%$. The two non-local functionals also give an improvement over LDA, but the reduction in error is not as great for diamond as it was for graphite.

In conclusion, we find that the dispersion-corrected functionals are all capable of correctly describing the ground state structural properties of both diamond and graphite. Overall, we suggest that the best functional to simultaneously describe both graphite and diamond bulk properties is

\footnotetext{
${ }^{3}$ Our theoretical calculations cannot reproduce the experimentally-found phase transition, and any associated changes in the lattice parameters, which are known to occur in a pressure range between $11 \mathrm{GPa}$ and $19 \mathrm{GPa}$ at room temperature. Therefore, the comparison to experiment makes sense up until these pressures. Beyond these pressures, experiment shows an increase in both the $a$ and $c$ lattice parameters compared to calculated values.
} 
Table 4.1: Bulk properties of Graphite $0 \mathrm{GPa}$. $\mathrm{B}_{0}$ and $\mathrm{B}_{0}^{\prime}$ were obtained using Murnaghan equation of state. Values $a_{0}$ and $c_{0}$ are provided in $\AA$, while $V_{0}$ is in $\AA^{3}, \mathrm{~B}_{0}$ is in $\mathrm{GPa}$, and both $\mathrm{B}_{0}^{\prime}$ and $\mathrm{c}_{0} / \mathrm{a}_{0}$ are dimensionless. MARE stands for "mean absolute error", with lower values meaning better overall agreement with the experiment.
Table 4.2: Bulk properties of Diamond at $0 \mathrm{GPa}$. Same considerations apply as for table with Graphite bulk properties.
$\mathrm{PBE}+\mathrm{TS}+\mathrm{SCS}$, with an MARE of just $0.2 \%$, followed by the optB86b functional $(2.2 \%)$ and then the PBE+D3+BJ functional $(2.3 \%)$.

\begin{tabular}{lcccccc} 
Functional & $\mathrm{a}_{0}$ & $\mathrm{c}_{0}$ & $\mathrm{c}_{0} / \mathrm{a}_{0}$ & $\mathrm{~B}_{0}$ & $\mathrm{~V}_{0}$ & MARE \\
\hline LDA & 2.447 & 6.652 & 2.719 & 25.5 & 34.5 & 7.7 \\
PBE+D3 & 2.465 & 6.965 & 2.825 & 23.2 & 36.7 & 10.8 \\
PBE+D3+BJ & 2.465 & 6.745 & 2.736 & 30.4 & 35.5 & 4.2 \\
PBE+TS & 2.459 & 6.690 & 2.721 & 53.5 & 35.0 & 12.3 \\
PBE+TS+SCS & 2.462 & 6.707 & 2.725 & 36.1 & 35.4 & 0.1 \\
rev-vdW-DF2 & 2.464 & 6.608 & 2.682 & 44.7 & 34.6 & 6.8 \\
optB86b & 2.466 & 6.620 & 2.685 & 33.8 & 34.9 & 2.3 \\
Exp & 2.462 & 6.707 & 2.724 & $34-42$ & 35.2 & - \\
Exp [126] & - & - & - & $36 \pm 1$ & - & - \\
Exp [127] & 2.460 & 6.700 & 2.724 & - & 35.1 & - \\
\hline
\end{tabular}

\begin{tabular}{lcccc} 
Functional & $\mathrm{a}_{0}$ & $\mathrm{~B}_{0}$ & $\mathrm{~V}_{0}$ & MARE \\
\hline LDA & 3.535 & 467.0 & 11.04 & 3.2 \\
PBE+D3 & 3.564 & 442.4 & 11.32 & 0.1 \\
PBE+D3+BJ & 3.557 & 445.3 & 11.25 & 0.4 \\
PBE+TS & 3.552 & 443.8 & 11.21 & 0.3 \\
PBE+TS+SCS & 3.563 & 439.8 & 11.32 & 0.4 \\
rev-vdW-DF2 & 3.567 & 421.1 & 11.36 & 2.5 \\
optB86b & 3.569 & 424.2 & 11.38 & 2.1 \\
Exp & 3.567 & 443.0 & 11.35 & - \\
\hline
\end{tabular}

\section{3 .2}

\section{Elastic constants}

Given that the values of some of the elastic constants are small, or zero, we cannot use the same metric as for bulk properties and instead define the $M A P E$ as:

$$
M A P E=\frac{100}{n} \sum_{t=1}^{n}\left|\frac{V_{\text {exp }}-V_{\text {theo }}}{E_{\text {exp }}}\right|
$$

where $n$ is the number of elastic constants considered, $V_{\text {exp }}$ is the experimental value, $V_{\text {theo }}$ is the theoretical value, and $E_{\text {exp }}$ is the experimental error. Within this definition, values within the experimental range gives errors between 0 and 1 .

Table 4.3 shows the calculated elastic constants of graphite at $0 \mathrm{GPa}$ with the different dispersion corrected functionals and compared to experiment. We find that the elastic constants of graphite are best described by the two non-local functionals, rev-vdW-DF2 and optB86b, which both give results 
within the experimental range. The largest error is given by the $\mathrm{PBE}+\mathrm{D} 3$ functional (2.6\%), followed by the two Tkatchenko-Scheffler functionals. D3+BJ and LDA give similar errors of approximately $1.5 \%$.

\begin{tabular}{lccccccc} 
Functional & $C_{11}$ & $C_{12}$ & $C_{13}$ & $C_{33}$ & $C_{44}$ & $C_{66}$ & MAPE \\
\hline LDA & 1097 & 209 & -2.4 & 31 & 5.1 & 444 & 1.5 \\
PBE+D3 & 1040 & 198 & -2.4 & 26 & 3.0 & 421 & 2.6 \\
PBE+D3+BJ & 1095 & 218 & -2.6 & 34 & 4.2 & 439 & 1.6 \\
PBE+TS & 1071 & 193 & -7.2 & 66 & 5.5 & 439 & 2.5 \\
PBE+TS+SCS & 1061 & 194 & -7.0 & 47 & 4.9 & 434 & 2.3 \\
rev-vdWDF2 & 1089 & 167 & -3.7 & 40 & 5.3 & 461 & 1.0 \\
optB86b & 1087 & 166 & -3.4 & 42 & 5.5 & 461 & 1.0 \\
Exp [126] & $1109 \pm 16$ & $139 \pm 36$ & $0 \pm 3$ & $38.7 \pm 7$ & $5.0 \pm 3$ & $485 \pm 10$ & \\
\hline
\end{tabular}

Diamond elastic constants are reported in table 4.4. LDA unsurprisingly yields substantial disagreement with experiment, with an absolute error of 7.8\%. The best results in terms of comparison with experiment are obtained with the $C_{6}$ corrected functionals with errors of between $1.7 \%$ and $2.8 \%$, while the non-local functionals do not perform as well with errors of almost $5 \%$.

\begin{tabular}{lcccc} 
Functional & $C_{11}$ & $C_{12}$ & $C_{44}$ & MAPE \\
\hline LDA & 1102 & 150 & 605 & 7.8 \\
PBE+D3 & 1064 & 132 & 579 & 1.7 \\
PBE+D3+BJ & 1072 & 132 & 588 & 2.7 \\
PBE+TS & 1075 & 128 & 588 & 2.2 \\
PBE+TS+SCS & 1055 & 132 & 574 & 2.8 \\
rev-vdWDF2 & 1052 & 128 & 562 & 4.7 \\
optB86b & 1052 & 129 & 564 & 4.5 \\
Exp [128] & $1079 \pm 5$ & $124 \pm 5$ & $578 \pm 2$ \\
\hline
\end{tabular}

The best functional for describing simultaneously the elastic constants of both graphite and diamond is the PBE+D3+BJ, although the other $C_{6}$ functionals perform reasonably well and the non-local functional perform worse, albeit giving an improved description compared to LDA. These results are in good agreement with other theoretical works $[129,130]$
Table 4.3: Elastic constants of Graphite at $0 \mathrm{GPa}$. All values are in GPa. MAPE stands for "mean absolute partial error". Lower values in MAPE represent higher agreement with the experimental data

Table 4.4: Elastic constants of Diamond at $0 \mathrm{GPa}$. All values are in GPa. MAPE stands for "mean absolute partial error". Lower values in MAPE represent higher agreement with the experimental data 
Figure 4.11: Comparison of calculated graphite pressure dependence of lattice parameters $c$ and $a$ with the experimental data in the pressure range from 0 up to $30 \mathrm{GPa}$. Solid lines represent our calculations using various van der Waals corrections.
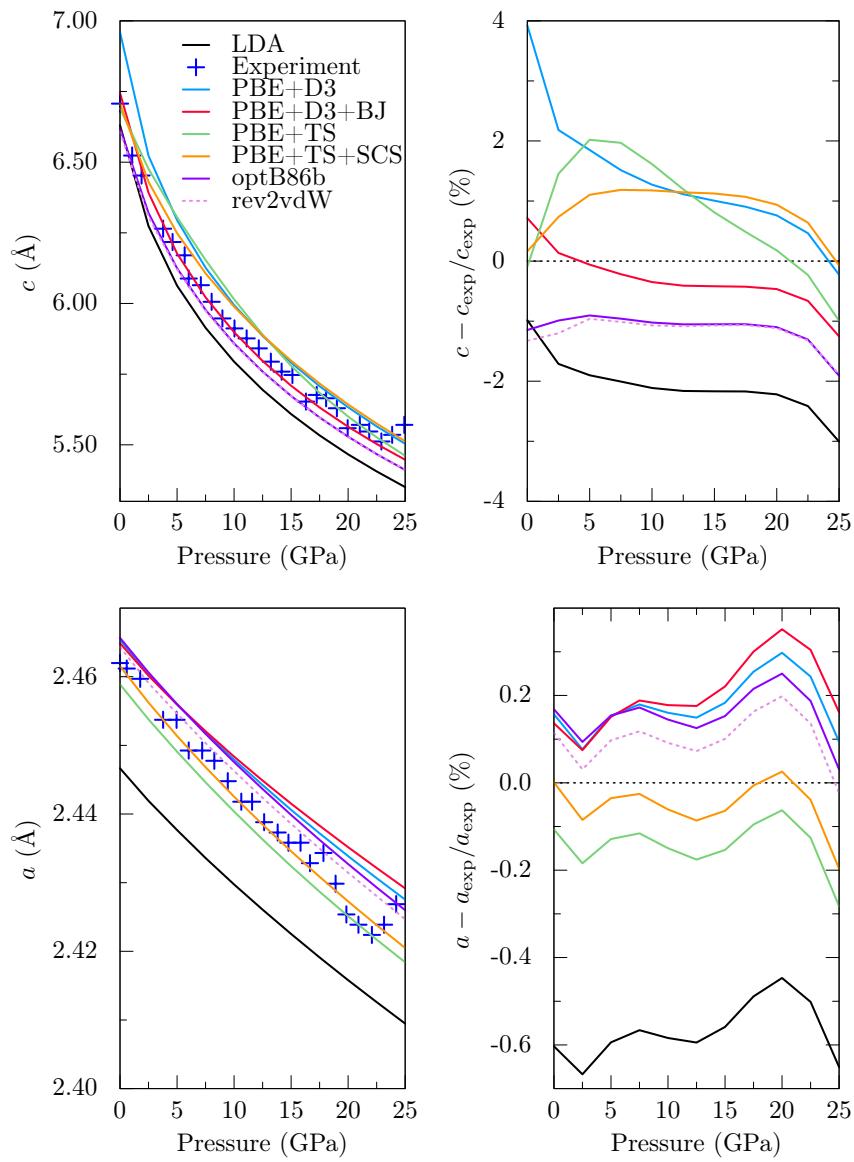
Figure 4.12: Diamond lattice constant $a$ as a function of pressure. Solid lines represent our calculations using various van der Waals corrections.
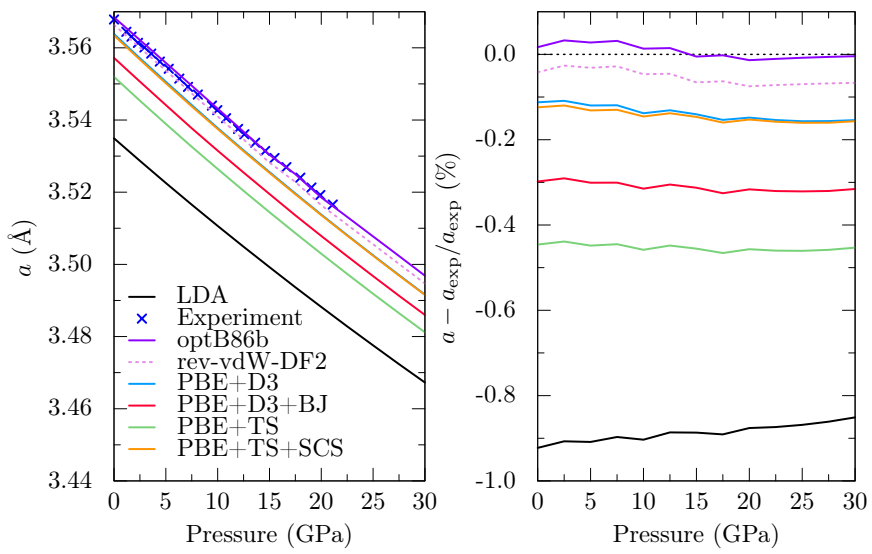



\section{Conclusion}

In this thesis I presented a description of theoretical methods and computational algorithms that allow to study materials at extreme conditions, namely high pressure and high temperatures. I presented a description of the methods used, and provided a detailed algorithms of their applications, with example results. However, a research is finished not when there is nothing left to do, but rather when the time runs out.

The highly efficient algorithm of free energy calculations that is based on temperature-dependent effective potential method can be extended and improved to have fewer manual steps. The similar idea of force constants fitting can be generalized over a grid of $T-V$ points which should allow to build a Helmholtz energy surface using temperature-dependent effective potential method with relative ease. The iron at the inner Earth's core conditions should be studied further with inclusion of additional structural phases, for example, body-centered cubic structure. Moreover, including iron-based alloys into consideration would further improve the power of the theoretical predictions.

The finite deformations algorithm for calculation of elastic constants could be automated and should be generalized further for the cases of crystal symmetries other than cubic. The possibility of electronic topological transition due to applied deformation in body-centered cubic Tantalum and Niobium due should be investigated further, possibly with the help of all-electron methods.

The work on graphite could be expanded if one goes beyond static calculations over to finite temperature simulations to study graphite-diamond transition under pressure. Overall, materials at extreme conditions provide a lot of interesting scientific problems and opportunities for a beautiful solutions and applications. 



\section{Bibliography}

[1] Daan Frenkel and Berend Smit. Understanding molecular simulation : from algorithms to applications. Academic Press, San Diego, 2002.

[2] E. Schrödinger. An undulatory theory of the mechanics of atoms and molecules. Physical Review, 28(6):10491070, dec 1926.

[3] W. Kohn and L. J. Sham. Self-consistent equations including exchange and correlation effects. Physical Review, 140(4A):A1133-A1138, nov 1965.

[4] P. Hohenberg and W. Kohn. Inhomogeneous electron gas. Physical Review, 136(3B):B864-B871, nov 1964.

[5] Gordon E. Moore. Cramming more components onto integrated circuits, reprinted from electronics, volume 38, number 8, april 19, 1965, pp.114 ff. IEEE Solid-State Circuits Newsletter, 20(3):33-35, sep 2006.

[6] Sunway taihulight - sunway mpp, sunway sw26010 260c 1.45ghz, sunway. https://www.top500.org/system/ 178764 .

[7] The top500 project. http://www.top500.org/.

[8] E. Anderson, Z. Bai, C. Bischof, S. Blackford, J. Demmel, J. Dongarra, J. Du Croz, A. Greenbaum, S. Hammarling, A. McKenney, and D. Sorensen. LAPACK Users' Guide. Society for Industrial and Applied Mathematics, Philadelphia, PA, third edition, 1999.

[9] Bengt Gustafsson, Lars Rydén, Gunnar Tibell, and Peter Wallensteen. The uppsala code of ethics for scientists. fournal of Peace Research, 21(4):311-316, 1984.

[10] Brendan Gregg. Systems Performance: Enterprise and the Cloud. Prentice Hall, 1st editio edition, 2013.

[11] Felix A. Faber, Alexander Lindmaa, O. Anatole Von Lilienfeld, and Rickard Armiento. Machine Learning Energies of 2 Million Elpasolite (ABC2D6) Crystals. Physical Review Letters, 117(13):2-7, 2016.

[12] Aaron Gilad Kusne, Tieren Gao, Apurva Mehta, Liqin Ke, Manh Cuong Nguyen, Kai-Ming Ho, Vladimir Antropov, Cai-Zhuang Wang, Matthew J. Kramer, Christian Long, and Ichiro Takeuchi. On-the-fly machinelearning for high-throughput experiments: search for rare-earth-free permanent magnets. Scientific Reports, 4:6367, sep 2014.

[13] G. E. Hinton. Reducing the Dimensionality of Data with Neural Networks. Science, 313(5786):504-507, jul 2006.

[14] Giuseppe Carleo and Matthias Troyer. Solving the quantum many-body problem with artificial neural networks. Science (New York, N.Y.), 355(6325):602-606, feb 2017.

[15] Pankaj Mehta and David J. Schwab. An exact mapping between the Variational Renormalization Group and Deep Learning. arXiv, pages 1-7, 2014.

[16] Zhenwei Li, James R. Kermode, and Alessandro De Vita. Molecular Dynamics with On-the-Fly Machine Learning of Quantum-Mechanical Forces. Physical Review Letters, 114(9):096405, mar 2015. 
[17] Anubhav Jain, Shyue Ping Ong, Geoffroy Hautier, Wei Chen, William Davidson Richards, Stephen Dacek, Shreyas Cholia, Dan Gunter, David Skinner, Gerbrand Ceder, and Kristin a. Persson. The Materials Project: A materials genome approach to accelerating materials innovation. APL Materials, 1(1):011002, 2013.

[18] Geoffroy Hautier, Chris Fischer, Virginie Ehrlacher, Anubhav Jain, and Gerbrand Ceder. Data mined ionic substitutions for the discovery of new compounds. Inorganic Chemistry, 50(2):656-663, jan 2011.

[19] R. Armiento et al. The high-throughput toolkit (httk). http://httk.openmaterialsdb.se/.

[20] Guoyin Shen, Ho-kwang Mao, Russell J. Hemley, Thomas S. Duffy, and Mark L. Rivers. Melting and Crystal Structure of Iron at High Pressures and Temperatures. Geophys. Res. Lett., 25(3):373-376, feb 1998.

[21] R. Boehler. Temperatures in the Earth's Core from Melting-point Measurements of Iron at High Static Pressures. Nature, 363(6429):534-536, jun 1993.

[22] J. Michael Brown and Robert G. McQueen. Phase Transitions, Grüneisen Parameter, and Elasticity for Shocked Iron between $77 \mathrm{GPa}$ and $400 \mathrm{GPa}$. F. Geophys. Res., 91(B7):7485, 1986.

[23] Jeffrey H Nguyen and Neil C Holmes. Melting of Iron at the Physical Conditions of the Earth's Core. Nature, 427(6972):339-42, jan 2004.

[24] C. Yoo, N. Holmes, M. Ross, D. Webb, and C. Pike. Shock Temperatures and Melting of Iron at Earth Core Conditions. Phys. Rev. Lett., 70(25):3931-3934, jun 1993.

[25] J. Kübler. Metastable Magnetic Ground-State of HCP-Fe. Solid State Commun., 72(7):631-633, nov 1989.

[26] A. B. Belonoshko. Equation of State for $\varepsilon$-Iron at High Pressures and Temperatures. Condens. Matter Phys., 13(2):23605, 2010.

[27] B a Buffett and H R Wenk. Texturing of the Earth's Inner Core by Maxwell Stresses. Nature, 413(6851):60-63, 2001.

[28] R. E. Cohen and S. Mukherjee. Non-collinear Magnetism in Iron at High Pressures. Phys. Earth Planet. Inter, 143(1-2):445-453, 2004.

[29] S. Gilder. Magnetic Properties of Hexagonal Closed-Packed Iron Deduced from Direct Observations in a Diamond Anvil Cell. Science, 279(5347):72-74, 1998.

[30] Jung-fu Lin, Dion L Heinz, and Andrew J Campbell. Iron-Silicon Alloy in Earth's Core? Science, 295(January):313-315, 2002.

[31] S. Merkel. Raman Spectroscopy of Iron to 152 Gigapascals: Implications for Earth's Inner Core. Science, 288(5471):1626-1629, 2000.

[32] G Steinle-Neumann, L Stixrude, R E Cohen, and O Gülseren. Elasticity of Iron at the Temperature of the Earth's Inner Core. Nature, 413(6851):57-60, sep 2001.

[33] Lars Stixrude and R. E. Cohen. High-pressure elasticity of iron and anisotropy of earth's inner core. Science, 267(5206):1972-1975, 1995.

[34] J. Söderlind, P.and Moriarty and J. M. Wills. First-principles Theory of Iron up to Earth-Core Pressures: Structural, Vibrational, and Elastic Properties. Phys. Rev. B, 53(21):14063-14072, jun 1996.

[35] S. Qiu and P. Marcus. Elasticity of HCP Nonmagnetic Fe under Pressure. Phys. Rev. B, 68(5):054103, aug 2003.

[36] L Vočadlo, D Alfè, M.J Gillan, and G.David Price. The Properties of Iron under Core Conditions from First Principles Calculations. Phys. Earth Planet. Inter., 140(1-3):101-125, nov 2003. 
[37] C. Asker, L. Vitos, and I. A. Abrikosov. Elastic Constants and Anisotropy in FeNi Alloys at High Pressures from First-principles Calculations. Phys. Rev. B, 79(21):214112, jun 2009.

[38] Krisztina Kádas, Levente Vitos, and Rajeev Ahuja. Elastic Properties of Iron-rich HCP Fe-Mg Alloys up to Earth's Core Pressures. Earth Planet. Sci. Lett., 271(1-4):221-225, jul 2008.

[39] Lidunka Vočadlo, David P. Dobson, and Ian G. Wood. Ab initio Calculations of the Elasticity of HCP-Fe as a Function of Temperature at Inner-core Pressure. Earth Planet. Sci. Lett., 288(3-4):534-538, nov 2009.

[40] Gerd Steinle-Neumann and R E Cohen. Comment on 'On the Importance of the Free Energy for Elasticity under Pressure'. F. Phys.: Condens. Matter, 16(47):8783-8786, dec 2004.

[41] C. Bercegeay and S. Bernard. First-principles Equations of State and Elastic Properties of Seven Metals. Phys. Rev. B, 72(21):214101, dec 2005.

[42] O. Hellman, I. A. Abrikosov, and S. I. Simak. Lattice dynamics of anharmonic solids from first principles. Phys. Rev. B, 84:180301, nov 2011.

[43] Olle Hellman, Peter Steneteg, I. A. Abrikosov, and S. I. Simak. Temperature Dependent Effective Potential Method for Accurate Free Energy Calculations of Solids. Phys. Rev. B, 87(10):104111, mar 2013.

[44] Agnès Dewaele, Marc Torrent, Paul Loubeyre, and Mohamed Mezouar. Compression curves of transition metals in the Mbar range: Experiments and projector augmented-wave calculations. Physical Review B Condensed Matter and Materials Physics, 78(10):1-13, 2008.

[45] T. Lukinov, S. I. Simak, and A. B. Belonoshko. Sound velocity in shock compressed molybdenum obtained by ab initio molecular dynamics. Physical Review B, 92(6):060101, aug 2015.

[46] Leonid Dubrovinsky, Natalia Dubrovinskaia, Vitali B Prakapenka, and Artem M Abakumov. Implementation of micro-ball nanodiamond anvils for high-pressure studies above 6 Mbar. Nature communications, 3:1163, jan 2012.

[47] L Dubrovinsky, N Dubrovinskaia, E Bykova, M Bykov, V Prakapenka, C Prescher, K Glazyrin, H-P Liermann, M Hanfland, M Ekholm, Q Feng, L V Pourovskii, M I Katsnelson, J M Wills, and I A Abrikosov. The most incompressible metal osmium at static pressures above 750 gigapascals. Nature, 525(7568):226-9, September 2015.

[48] Xiaoli Huang, Fangfei Li, Qiang Zhou, Yue Meng, Konstantin D Litasov, Xin Wang, Bingbing Liu, and Tian Cui. Thermal equation of state of Molybdenum determined from in situ synchrotron X-ray diffraction with laser-heated diamond anvil cells. Scientific reports, 6(August 2015):19923, 2016.

[49] Yang Ding, Rajeev Ahuja, Jinfu Shu, Paul Chow, Wei Luo, and Ho kwang Mao. Structural phase transition of vanadium at $69 \mathrm{GPa}$. Physical Review Letters, 98(8), feb 2007.

[50] C L Lu, C P Chang, Y C Huang, J M Lu, C C Hwang, and M F Lin. Low-energy electronic properties of the AB-stacked few-layer graphites. Journal of Physics: Condensed Matter, 18(26):5849-5859, jun 2006.

[51] Jue Wang, Federica Coppari, Raymond F. Smith, Jon H. Eggert, Amy E. Lazicki, Dayne E. Fratanduono, J. Ryan Rygg, Thomas R. Boehly, Gilbert W. Collins, and Thomas S. Duffy. X-ray diffraction of molybdenum under ramp compression to $1 \mathrm{TPa}$. Physical Review B, 94(10):104102, sep 2016.

[52] Xiao gang Wen. Quantum field theory of many-body systems, 2004.

[53] G. Pilania, J. E. Gubernatis, and T. Lookman. Structure classification and melting temperature prediction in octet AB solids via machine learning. Physical Review B, 91(21), jun 2015. 
[54] L Schimka, J Harl, A Stroppa, A Grüneis, M Marsman, F Mittendorfer, and G Kresse. Accurate surface and adsorption energies from many-body perturbation theory. Nature Materials, 9(9):741-744, 2010.

[55] Thomas Olsen and Kristian S Thygesen. Random phase approximation applied to solids, molecules, and graphene-metal interfaces: From van der waals to covalent bonding. Physical Review B, 87(7):075111, 2013.

[56] W. M. C. Foulkes, L. Mitas, R. J. Needs, and G. Rajagopal. Quantum monte carlo simulations of solids. Reviews of Modern Physics, 73(1):33-83, jan 2001.

[57] Celso R C Rêgo, Luiz N Oliveira, Polina Tereshchuk, and Juarez L F Da Silva. Comparative study of van der Waals corrections to the bulk properties of graphite. Fournal of Physics: Condensed Matter, 27(41):415502, 2015.

[58] A. Bosak, M. Krisch, A. Chumakov, I.A. Abrikosov, and L. Dubrovinsky. Possible artifacts in inferring seismic properties from X-ray data. Physics of the Earth and Planetary Interiors, 260:14-19, nov 2016.

[59] L. H. Thomas. The calculation of atomic fields. Mathematical Proceedings of the Cambridge Philosophical Society, 23(05):542, jan 1927.

[60] Enrico Fermi. Un metodo statistico per la determinazione di alcune priorieta dell'atome. Rend. Accad. Naz. Lincei, 6(602-607):32, 1927.

[61] J. C. Slater. Atomic shielding constants. Physical Review, 36(1):57-64, jul 1930.

[62] Richard Martin. Electronic structure : basic theory and practical methods. Cambridge University Press, Cambridge, UK New York, 2004.

[63] Ann E Mattsson, Peter A Schultz, Michael P Desjarlais, Thomas R Mattsson, and Kevin Leung. Designing meaningful density functional theory calculations in materials science-a primer. Modelling and Simulation in Materials Science and Engineering, 13(1):R1-R31, nov 2004.

[64] C. S. Wang, B. M. Klein, and H. Krakauer. Theory of magnetic and structural ordering in iron. Physical Review Letters, 54(16):1852-1855, apr 1985.

[65] John P. Perdew, J. A. Chevary, S. H. Vosko, Koblar A. Jackson, Mark R. Pederson, D. J. Singh, and Carlos Fiolhais. Atoms, molecules, solids, and surfaces: Applications of the generalized gradient approximation for exchange and correlation. Physical Review B, 46(11):6671-6687, sep 1992.

[66] John P. Perdew, Kieron Burke, and Matthias Ernzerhof. Generalized Gradient Approximation Made Simple. Phys. Rev. Lett., 77(18):3865-3868, oct 1996.

[67] N.W. Ashcroft and N.D. Mermin. Solid State Physics. Saunders College, Philadelphia, 1976.

[68] J.S. Blakemore. Solid state physics. Cambridge University Press, Cambridge, Great Britain, 2nd edition, 1985.

[69] P. E. Blöchl. Projector Augmented-Wave Method. Phys. Rev. B, 50(24):17953, dec 1994.

[70] G. Kresse and D. Joubert. From Ultrasoft Pseudopotentials to the Projector Augmented-Wave Method. Phys. Rev. B, 59(3):1758, jan 1999.

[71] G. Kresse and J. Furthmüller. Efficient iterative schemes for ab initio total-energy calculations using a planewave basis set. Physical Review B, 54(16):11169, October 1996.

[72] G. Kresse and J. Furthmüller. Efficient iterative schemes for ab initio total-energy calculations using a planewave basis set. Phys. Rev. B, 54:11169-11186, oct 1996.

[73] G. Kresse and J. Hafner. Ab initio molecular-dynamics simulation of the liquid-metal-amorphoussemiconductor transition in germanium. Phys. Rev. B, 49:14251-14269, may 1994.

[74] G. Kresse and J. Hafner. Ab initio molecular dynamics for liquid metals. Phys. Rev. B, 47:558-561, Jan 1993. 
[75] G. Kresse and J. Furthmüller. Efficiency of ab-initio total energy calculations for metals and semiconductors using a plane-wave basis set. Comput. Mater. Sci., 6(1):15 - 50, 1996.

[76] Hendrik J. Monkhorst and James D. Pack. Special Points for Brillouin-Zone Integrations. Phys. Rev. B, 13(12):5188-5192, jun 1976.

[77] Stefan Grimme. Semiempirical GGA-type density functional constructed with a long-range dispersion correction. fournal of Computational Chemistry, 27(15):1787-1799, nov 2006.

[78] Stefan Grimme, Jens Antony, Stephan Ehrlich, and Helge Krieg. A consistent and accurate ab initio parametrization of density functional dispersion correction (DFT-D) for the 94 elements $\mathrm{H}-\mathrm{Pu}$. Fournal of Chemical Physics, 132(15), 2010.

[79] Stefan Grimme, Stephan Ehrlich, and Lars Goerigk. Effect of the damping function in dispersion corrected density functional theory. fournal of Computational Chemistry, 32(7):1456-1465, may 2011.

[80] Axel D. Becke and Erin R. Johnson. Exchange-hole dipole moment and the dispersion interaction. The fournal of Chemical Physics, 122(15):154104, 2005.

[81] Axel D. Becke and Erin R. Johnson. A density-functional model of the dispersion interaction. The fournal of Chemical Physics, 123(15):154101, 2005.

[82] Erin R. Johnson and Axel D. Becke. A post-Hartree-Fock model of intermolecular interactions. The fournal of Chemical Physics, 123(2):024101, 2005.

[83] Erin R. Johnson and Axel D. Becke. A post-Hartree-Fock model of intermolecular interactions: Inclusion of higher-order corrections. The fournal of Chemical Physics, 124(17):174104, 2006.

[84] Alexandre Tkatchenko and Matthias Scheffler. Accurate Molecular Van Der Waals Interactions from GroundState Electron Density and Free-Atom Reference Data. Physical Review Letters, 102(7):073005, feb 2009.

[85] Alexandre Tkatchenko, Robert A. DiStasio, Roberto Car, and Matthias Scheffler. Accurate and Efficient Method for Many-Body van der Waals Interactions. Physical Review Letters, 108(23):236402, jun 2012.

[86] Kyuho Lee, Éamonn D. Murray, Lingzhu Kong, Bengt I. Lundqvist, and David C. Langreth. Higher-accuracy van der Waals density functional. Physical Review B, 82(8):081101, aug 2010.

[87] T. Thonhauser, Valentino R. Cooper, Shen Li, Aaron Puzder, Per Hyldgaard, and David C. Langreth. Van der Waals density functional: Self-consistent potential and the nature of the van der Waals bond. Physical Review $B, 76(12): 125112$, sep 2007.

[88] Jiři Klimeš, David R Bowler, and Angelos Michaelides. Chemical accuracy for the van der Waals density functional. Journal of Physics: Condensed Matter, 22(2):022201, jan 2010.

[89] Oleg A. Vydrov and Troy Van Voorhis. Nonlocal van der Waals Density Functional Made Simple. Physical Review Letters, 103(6):063004, aug 2009.

[90] Yingkai Zhang and Weitao Yang. Comment on "Generalized Gradient Approximation Made Simple". Physical Review Letters, 80(4):890-890, jan 1998.

[91] A. D. Becke. Density functional calculations of molecular bond energies. The fournal of Chemical Physics, 84(8):4524, 1986.

[92] F. D. Murnaghan. The Compressibility of Media under Extreme Pressures. Proceedings of the National Academy of Sciences, 30(9):244-247, September 1944.

[93] Francis Birch. Finite Elastic Strain of Cubic Crystals. Physical Review, 71(11):809-824, June 1947. 
[94] P. Vinet, J. Ferrante, J. H. Rose, and J. R. Smith. Compressibility of Solids. J. Geophys. Res., 92(B9):9319, 1987.

[95] S. R. Bahn and K. W. Jacobsen. An object-oriented scripting interface to a legacy electronic structure code. Comput. Sci. Eng., 4(3):56-66, MAY-JUN 2002.

[96] Levente Vitos. Computational Quantum Mechanics for Materials Engineers: The EMTO Method and Applications. Springer London Ltd, 2007.

[97] Y Hiki. Higher Order Elastic Constants of Solids. Annual Review of Materials Science, 11(1):51-73, aug 1981.

[98] R.P. Feynman. The Feynman Lectures on Physics. Addison-Wesley Pub. Co., 1963.

[99] A. Van de Walle and G. Ceder. The Effect of Lattice Vibrations on Substitutional Alloy Thermodynamics. Rev. Mod. Phys., 74(1):11-45, 2002.

[100] Duanne C. Wallace. Thermodynamics of Crystals. Dover Publications, 1998.

[101] D. West and S. K. Estreicher. First-principles Calculations of Vibrational Lifetimes and Decay Channels: Hydrogen-related Modes in Si. Phys. Rev. Lett., 96(11), 2006.

[102] P. Steneteg, O. Hellman, O. Yu. Vekilova, N. Shulumba, F. Tasnádi, and I. A. Abrikosov. Temperature Dependence of TiN Elastic Constants from ab initio Molecular Dynamics Simulations. Phys. Rev. B, 87(9):094114, 2013.

[103] B. Grabowski, L. Ismer, T. Hickel, and J. Neugebauer. Ab initio up to the Melting Point: Anharmonicity and Vacancies in Aluminum. Phys. Rev. B, 79(13):134106, apr 2009.

[104] Thomas S. Duffy, Guoyin Shen, Jinfu Shu, Ho-Kwang Mao, Russell J. Hemley, and Anil K. Singh. Elasticity, shear strength, and equation of state of molybdenum and gold from x-ray diffraction under nonhydrostatic compression to $24 \mathrm{GPa}$. Journal of Applied Physics, 86(12):6729, 1999.

[105] Wei Liu, Qiong Liu, Matthew L. Whitaker, Yusheng Zhao, and Baosheng Li. Experimental and theoretical studies on the elasticity of molybdenum to $12 \mathrm{GPa}$. Journal of Applied Physics, 106(4):043506, 2009.

[106] Jeffrey H. Nguyen, Minta C. Akin, Ricky Chau, Dayne E. Fratanduono, W. Patrick Ambrose, Oleg V. Fat'yanov, Paul D. Asimow, and Neil C. Holmes. Molybdenum sound velocity and shear modulus softening under shock compression. Physical Review B, 89(17):174109, may 2014.

[107] R. S. Hixson and J. N. Fritz. Shock compression of tungsten and molybdenum. Fournal of Applied Physics, 71(4):1721, 1992.

[108] Xianwei Sha and R. E. Cohen. First-principles Thermal Equation of State and Thermoelasticity of HCP Fe at High Pressures. Phys. Rev. B, 81(9):094105, mar 2010.

[109] K. Glazyrin, L. V. Pourovskii, L. Dubrovinsky, O. Narygina, C. McCammon, B. Hewener, V. Schünemann, J. Wolny, K. Muffler, A. I. Chumakov, W. Crichton, M. Hanfland, V. B. Prakapenka, F. Tasnádi, M. Ekholm, M. Aichhorn, V. Vildosola, A. V. Ruban, M. I. Katsnelson, and I. A. Abrikosov. Importance of Correlation Effects in HCP Iron Revealed by a Pressure-Induced Electronic Topological Transition. Phys. Rev. Lett., 110(11):1$5,2013$.

[110] L. V. Pourovskii, J. Mravlje, M. Ferrero, O. Parcollet, and I. A. Abrikosov. Impact of Electronic Correlations on the Equation of State and Transport in $\mathcal{E}$-Fe. Phys. Rev. B, 90:155120, oct 2014.

[111] O.M. Krasilnikov, M.P. Belov, A.V. Lugovskoy, I.Yu. Mosyagin, and Yu.Kh. Vekilov. Elastic properties, lattice dynamics and structural transitions in molybdenum at high pressures. Computational Materials Science, 81:313-318, January 2014. 
[112] K.-H. Hellwege and O Madelung, editors. Numerical Data and Functional Relationships in Science and Technology, volume 18 of Group \{III\}: Crystal and Solid State Physics. Springer-Verlag, Berlin Heidelberg New York Tokyo, 1984.

[113] F F Voronov, V M Prohorov, E L Gromnickaya, and G G Ilina. Second and third order elastic moduli of molybdenum monocrystal. The Physics of Metals and Metallography, 45(6):94105, 1978.

[114] Yusheng Zhao, Andrew C Lawson, Jiangzhong Zhang, Bard I Bennett, and Robert B Von Dreele. Thermoelastic equation of state of molybdenum. Phys. Rev. B, 62(13):8766-8776, 2000.

[115] L. Koči, Y. Ma, a. Oganov, P. Souvatzis, and R. Ahuja. Elasticity of the superconducting metals V, Nb, Ta, Mo, and W at high pressure. Physical Review B, 77(21):214101, June 2008.

[116] O. Gülseren and R. E. Cohen. High-pressure thermoelasticity of body-centered-cubic tantalum. Physical Review $B, 65(6): 064103,2002$.

[117] Hyunchae Cynn and Choong-Shik Yoo. Equation of state of tantalum to 174 GPa. Physical Review B, 59(13):8526-8529, apr 1999.

[118] Michel Foata-Prestavoine, Grégory Robert, Marie-Hélène Nadal, and Stéphane Bernard. First-principles study of the relations between the elastic constants, phonon dispersion curves, and melting temperatures of bcc ta at pressures up to1000gpa. Physical Review B, 76(10), sep 2007.

[119] D Gerlich, S Hart, and D Whittal. The elastic network model reveals a consistent picture on intrinsic functional dynamics of type II restriction endonucleases. Physical Review B, 29(4):2142-2147, 1984.

[120] Keith J. Carroll. Elastic Constants of Niobium from $4.2^{\circ}$ to $300^{\circ} \mathrm{K}$. Fournal of Applied Physics, 36(11):3689, 1965.

[121] J. Trivisonno, S. Vatanayon, M. Wilt, J. Washick, and R. Reifenberger. Temperature dependence of the elastic constants of niobium and lead in the normal and superconducting states. Fournal of Low Temperature Physics, 12(1-2):153-169, 1973.

[122] A. Landa, P. Söderlind, A. V. Ruban, O. E. Peil, and L. Vitos. Stability in bcc transition metals: Madelung and band-energy effects due to alloying. Physical Review Letters, 103(December):1-4, 2009.

[123] Yvon Le Page and Paul Saxe. Symmetry-general least-squares extraction of elastic data for strained materials from ab initio calculations of stress. Physical Review B, 65(10):104104, feb 2002.

[124] Xifan Wu, David Vanderbilt, and D. R. Hamann. Systematic treatment of displacements, strains, and electric fields in density-functional perturbation theory. Physical Review B, 72(3):035105, jul 2005.

[125] Y Wang, J E Panzik, B Kiefer, and K K Lee. Crystal structure of graphite under room-temperature compression and decompression. Sci Rep, 2:520, 2012.

[126] Alexey Bosak, Michael Krisch, Marcel Mohr, Janina Maultzsch, and Christian Thomsen. Elasticity of singlecrystalline graphite: Inelastic x-ray scattering study. Physical Review B, 75(15):153408, apr 2007.

[127] Y. Baskin and L. Meyer. Lattice Constants of Graphite at Low Temperatures. Physical Review, 100(2):544-544, oct 1955 .

[128] HJ McSkimin and P Andreatch Jr. Elastic moduli of diamond as a function of pressure and temperature. Fournal of Applied Physics, 43(7):2944-2948, 1972.

[129] G Savini, YJ Dappe, Sven Öberg, J-C Charlier, MI Katsnelson, and A Fasolino. Bending modes, elastic constants and mechanical stability of graphitic systems. Carbon, 49(1):62-69, 2011.

[130] Nicolas Mounet and Nicola Marzari. First-principles determination of the structural, vibrational and thermodynamic properties of diamond, graphite, and derivatives. Physical Review B, 71(20):205214, may 2005. 



\section{List of figures}

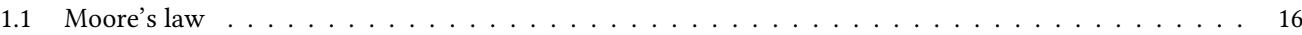

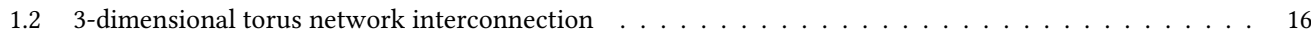

1.3 Geometric structure of AB-stacked graphite . . . . . . . . . . . . . . . . . . . . . . 19

2.1 DFT: replacing one many-body problem with many single-body problems . . . . . . . . . . . 27

2.2 Pseudopotential and cut-off radius . . . . . . . . . . . . . . . . . . . . . . . . 29

$2.3 \Delta E\left(\delta^{2}\right)$ in compressed bcc $\mathrm{Nb}$ with lattice constant $a=2.8 \AA^{3} \ldots \ldots \ldots$

2.4 effect of $\eta$ range on energy fit in deformed bcc $\mathrm{Nb} \ldots \ldots \ldots \ldots$

2.5 Non-linear behavior of $U_{\mathrm{uc}}$ of hcp Fe as a function of volume $\ldots \ldots \ldots \ldots$

2.6 Force constants matrix component fitted over the irregular $T-V$ grid $\ldots \ldots \ldots 7$

$2.7 \Delta U$ fit over irregular $T-V$ grid $\ldots \ldots \ldots \ldots \ldots \ldots \ldots$

3.1 Helmholtz free energy calculation algorithm using TDEP . . . . . . . . . . . . . . . . . . 51

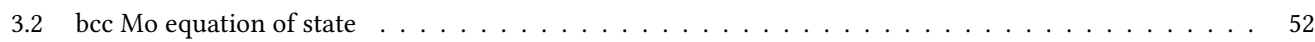

3.3 Energy dependency fit parameters as a function of $\eta$ at different pressures . . . . . . . . . . . . . 5 54

3.4 Elastic constants calculation algorithm using finite strain tensor . . . . . . . . . . . . . 55

4.1 Pressure in hcp Fe as a function of $V / V_{0}$ at $T=2000 \mathrm{~K} \ldots \ldots \ldots$

4.2 Second-order elastic constants of bcc Molybdenum as a function of pressure . . . . . . . . . . . . 59

4.3 Third-order elastic constants of bcc Molybdenum as a function of pressure . . . . . . . . . . . . 60

4.4 Second-order elastic constants of bcc Tantalum as a function of pressure . . . . . . . . . . . . 61

4.5 Third-order elastic constants of bcc Tantalum as a function of pressure . . . . . . . . . . . . . 62

4.6 Second-order elastic constants of bcc Niobium as a function of pressure . . . . . . . . . . . . . . 63

4.7 Band structure of bcc $\mathrm{Nb}$ at $0 \mathrm{GPa} \ldots \ldots \ldots \ldots \ldots$

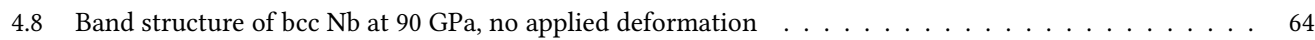

4.9 Behaviour of $\Delta E\left(\delta_{m}\right)$ and $\sigma\left(\delta_{m}\right)$ in bcc Niobium at 0,68 and $100 \mathrm{GPa} \ldots \ldots \ldots$

4.10 Band structure of bcc $\mathrm{Nb}$ under monoclinic deformation at $\mathrm{P}=68 \mathrm{GPa} \ldots \ldots$

4.11 Graphite lattice parameters $c$ and $a$ as function of pressure . . . . . . . . . . . . . 70

4.12 Diamond lattice constant $a$ as a function of pressure . . . . . . . . . . . . . . 71 



\section{Papers}

\section{List of papers and my contributions}

\section{Paper I}

$\mathrm{Ab}$ initio calculations of pressure-dependence of high-order elastic constants using finite deformations approach

I planned the project, carried out most of the calculations and performed a data analysis. I wrote most of the manuscript.

\section{Paper II}

Effect of dispersion corrections on graphite and diamond structure properties under pressure and their elastic constants

I took part in planning and executing the project. I wrote most of the manuscript, excluding section on enthalpy analysis with respect to stacking sequence.

\section{Paper III}

Highly Efficient Free Energy Calculations of Fe Equation of State Using Temperature-Dependent Effective Potential Method

I took part in planning and executing this project. All calculations and analysis were done by me. I wrote the majority of the manuscript.

\section{Paper IV}

Theoretical description of pressure-induced phase transitions: a case study of Ti-V alloys

I carried out theoretical description of temperature-induced dynamical stabilization of body-centered cubic Titanium and provided description of these results.

\section{Paper V}

Finite Temperature, Magnetic, and Many-Body Effects in Ab Initio Simulations of Alloy Thermodynamics

In this project I calculated phonon spectra of Ti using Temperature-Dependent effective potential and provided a corresponding subsection's text with computational details. 


\section{Papers not included in thesis}

1. Elastic constants of solids at high pressures.

Krasil'nikov, O. M., Vekilov, Y. K., and Mosyagin, I. Y.

(2012) Journal of Experimental and Theoretical Physics, 115(2), 237-241.

DOI:10.1134/S1063776112070096

2. Elastic phase transitions in metals at high pressures

Krasilnikov, O. M., Vekilov, Y. K., Mosyagin, I. Y., Isaev, E. I., and Bondarenko, N. G.

(2012) Journal of Physics-Condensed Matter, 24(19).

DOI:10.1088/0953-8984/24/19/195402

3. Structural transformations at high pressure in the refractory metals ( $\mathrm{Ta}, \mathrm{Mo}, \mathrm{V}$ )

Krasilnikov, O. M., Vekilov, Y. K., Lugovskoy, A. V., Mosyagin, I. Y., Belov, M. P., and Bondarenko, N. G. (2014) Journal of Alloys and Compounds, 586, S242-S245.

DOI:10.1016/j.jallcom.2013.05.151

4. Elastic properties, lattice dynamics and structural transitions in molybdenum at high pressures Krasilnikov, O. M., Belov, M. P., Lugovskoy, A. V., Mosyagin, I. Y., and Vekilov, Y. K.

(2014) Computational Materials Science, 81, 313-318.

DOI:10.1016/j.commatsci.2013.08.038 


\section{Papers}

The articles associated with this thesis have been removed for copyright reasons. For more details about these see:

http://urn.kb.se/resolve?urn=urn:nbn:se:liu:diva- 136447 